\title{
COGNITIVE FACTORS' INFLUENCE ON FRACTION DEVELOPMENT IN STUDENTS OF VARYING ABILITY LEVELS
}

\author{
A Dissertation \\ Presented to the Faculty of the Curry School of Education \\ University of Virginia \\ In Partial Fulfillment \\ of the Requirements for the Degree \\ Doctor of Philosophy
}

By

Andrew Bruce, Ph.D., M.A.T.

December 2014 


\section{ACKNOWLEDGEMENTS}

I would like to thank all of the people who have helped to make this dissertation a reality. To my wonderful wife Andy, thank you for all you sacrificed for this process to be possible. To my parents, your belief in me has meant a lot over the past several years. To the members of my committee; Patrick, Michael, and Robert, thank you for your time and guidance, your help has been invaluable. Finally, thank you to John, my advisor over the past four plus years. I hope to bring your kindness, patience, and excellence with me in my professional career. 


\begin{abstract}
The aim of this study was to evaluate the relationships between student attention, nonverbal reasoning, math achievement, and a variety of fraction outcomes, and to examine how those relationships vary for students of differing ability levels. This study provides initial evidence of the influence of nonverbal reasoning on conceptual understanding, estimation, and word problem set-up of fraction problems, and provides evidence that nonverbal reasoning may not be significantly linked to fraction calculation. This study also found that a more direct measure of student attention was significantly linked to all included fraction outcomes, though the relationship was not as strong as those found in previous studies using teacher ratings of attention. Furthermore, teacher ratings of student attention and results of the d2 Test of Attention explained very little of the same variance on fraction outcomes. The influence of cognitive factors seemed to hold steady across participants in all three ability groups. Participants with school identified disabilities showed significant deficits in the conceptual understanding of fractions even when controlling for general math ability. Essentially, students with identified disabilities separated themselves from those with math difficulties in their weak conceptual understanding of fractions. Students with math learning disabilities also scored significantly below their peers with math difficulties in nonverbal reasoning and word problem set-up.
\end{abstract}




\section{TABLE OF Contents}

COGNITIVE FACTORS' INFLUENCE ON FRACTION DEVELOPMENT IN STUDENTS OF VARYING ABILITY LEVELS.........I

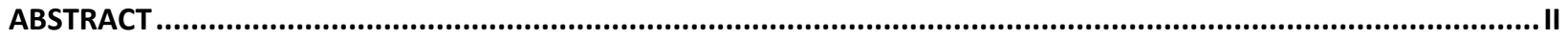

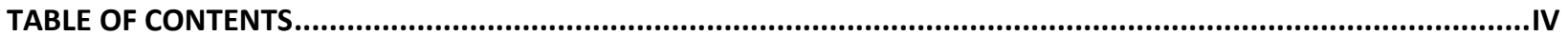

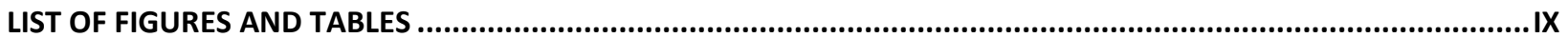

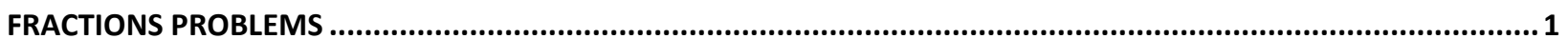

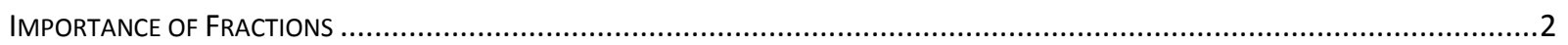

Importance of Fractions Within School .................................................................................................

Utility of Fractions Outside School ....................................................................................................

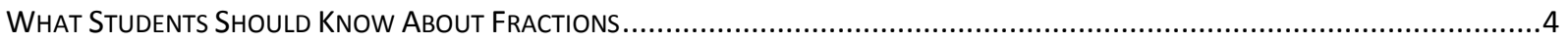

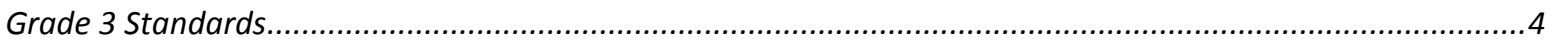

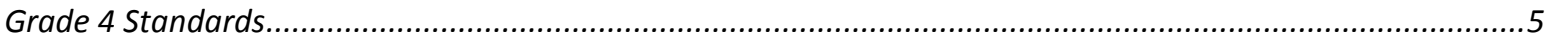

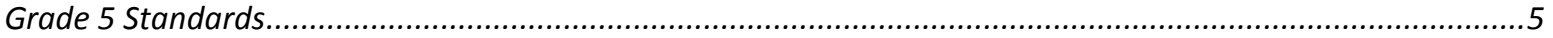

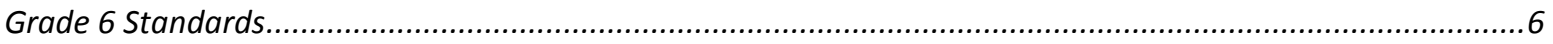

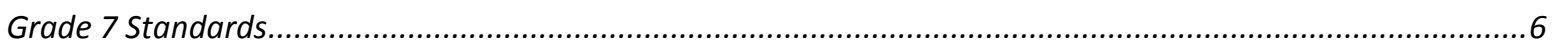

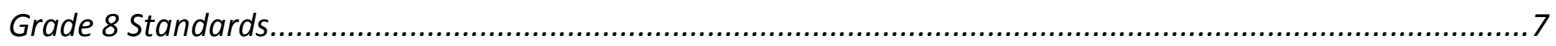

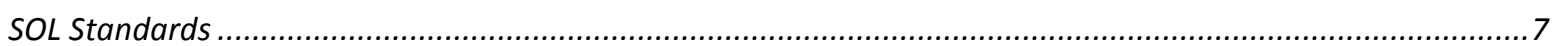

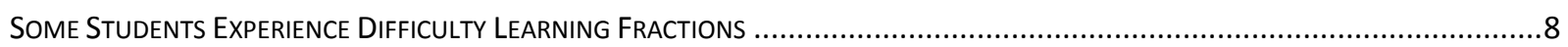

Student Difficulties in the Elementary Grades....................................................................................

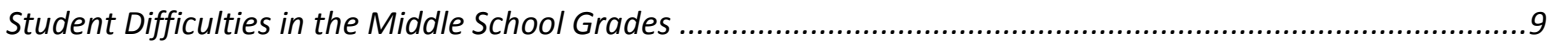

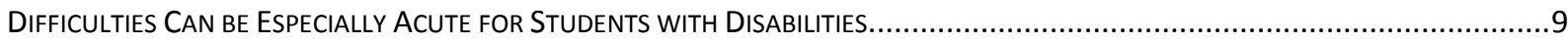

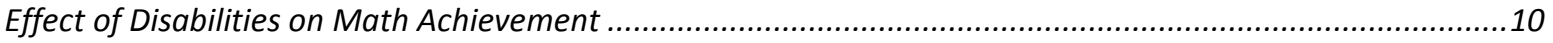

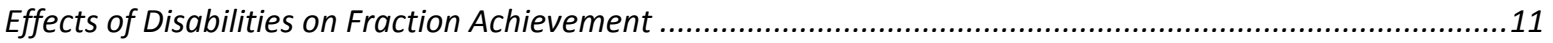

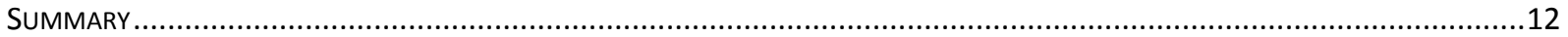

WHY DO STUDENTS STRUGGLE WITH FRACTIONS? .......................................................................... 


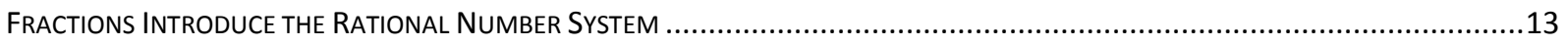

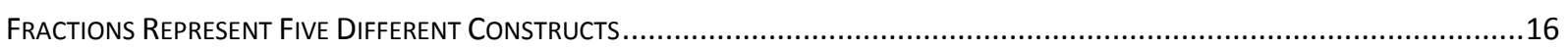

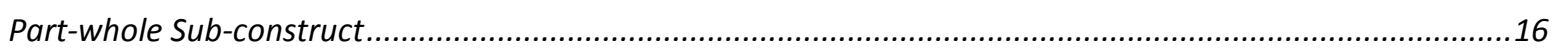

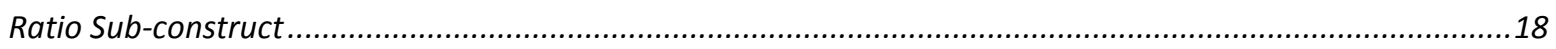

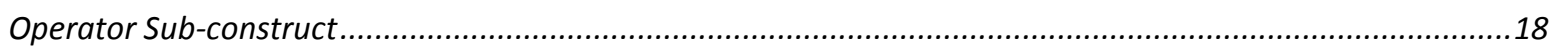

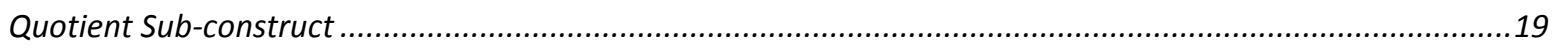

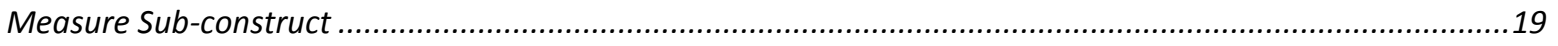

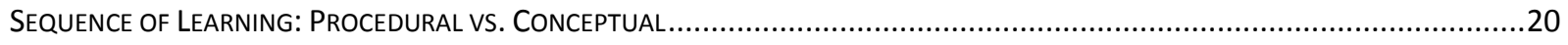

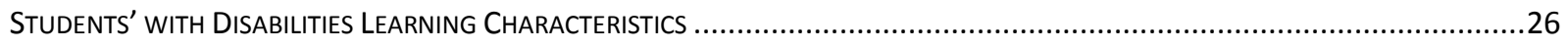

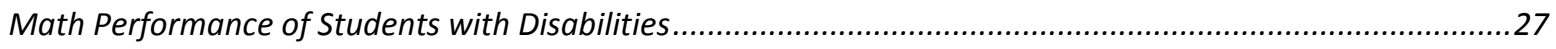

Fraction Performance of Students with Disabilities .....................................................................28

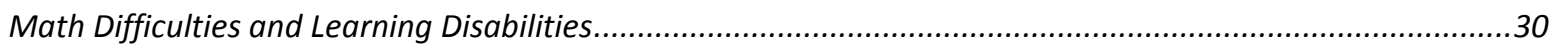

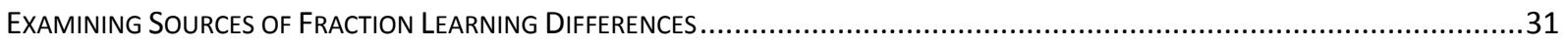

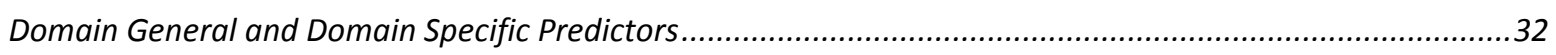

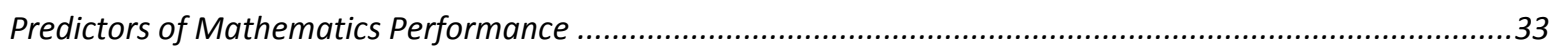

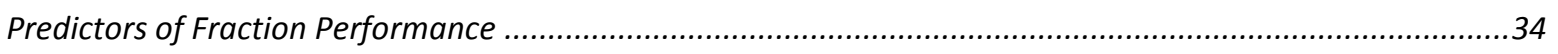

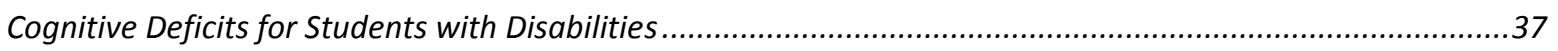

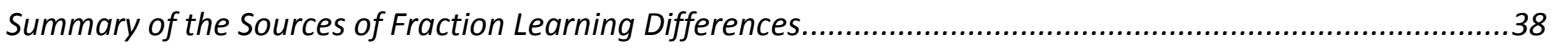

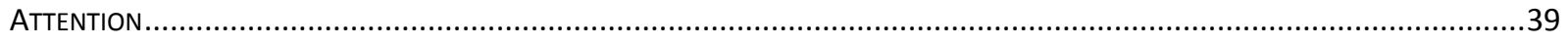

Attention as a Predictor of Fraction Performance .............................................................................

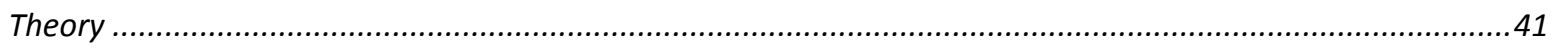

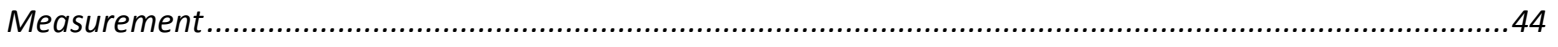

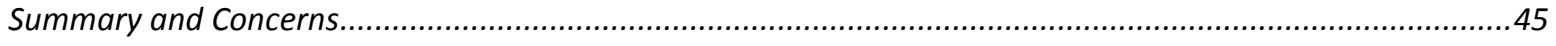

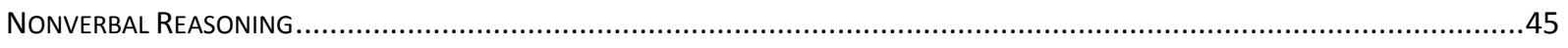

Nonverbal Reasoning as a Predictor of Fraction Performance .................................................................46

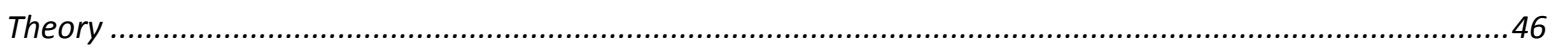

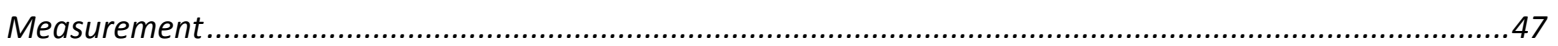




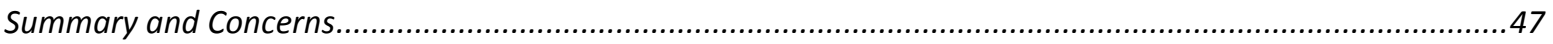

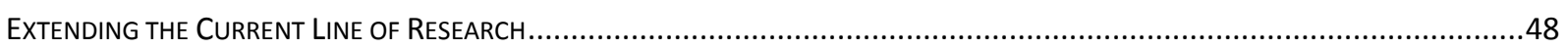

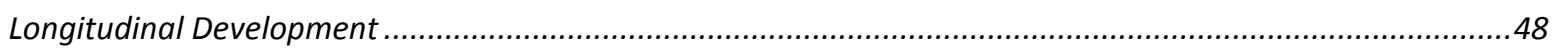

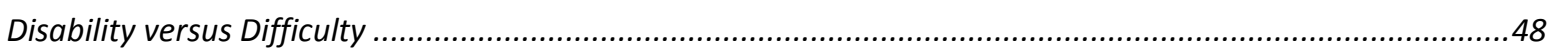

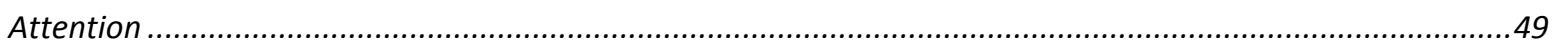

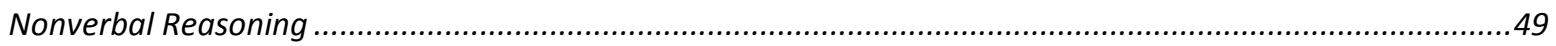

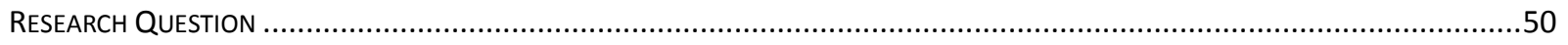

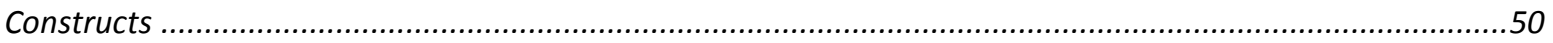

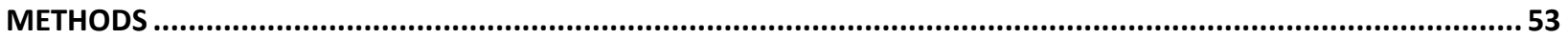

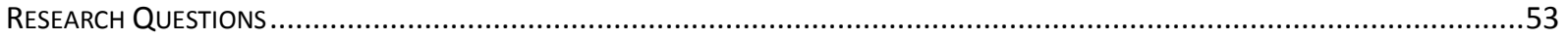

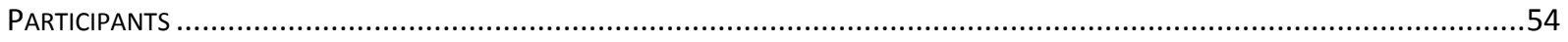

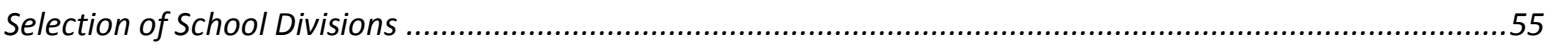

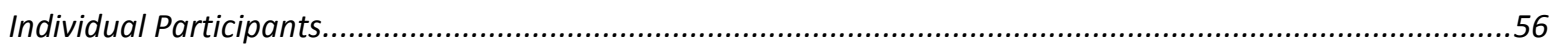

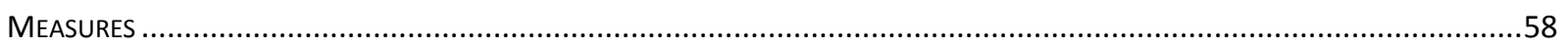

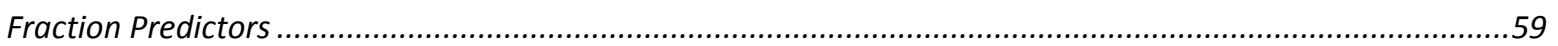

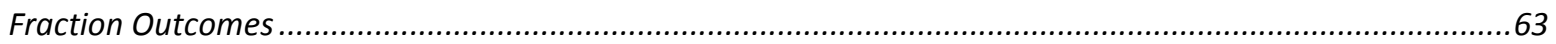

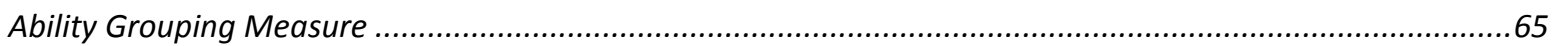

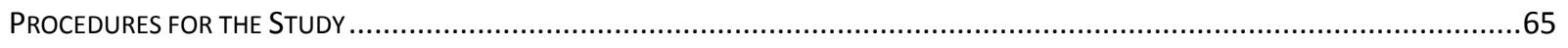

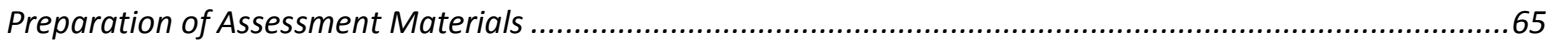

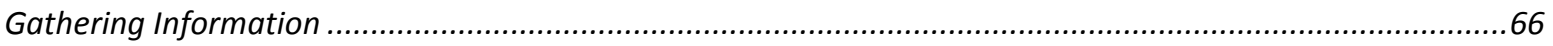

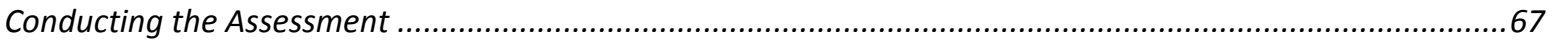

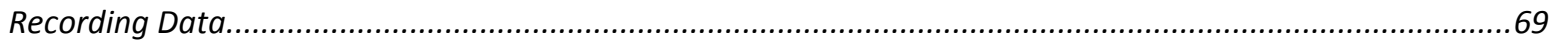

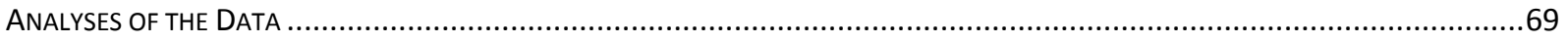

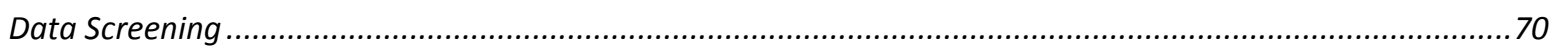

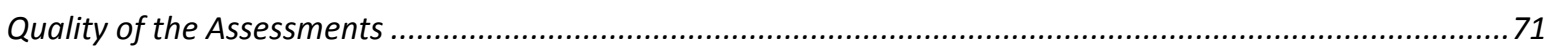

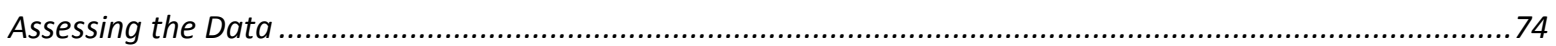


DATA SCREENING

Missing Data

Collinearity

Outliers.

Normality

Linearity and Homoscedasticity

Screening of Assessment Items .

RESULTS OF THE PATH ANALYSIS...

Ho: Domain-general (i.e., attention, nonverbal reasoning) and domain-specific (i.e., conceptual understanding of fractions) factors uniquely explain significant amounts of variance in fraction outcomes......

Attention

Nonverbal Reasoning.

Conceptual Understanding of Fractions.

Ho: There are significant differences in fraction outcomes between the fourth, fifth, and sixth grades.

Ho: There are significant differences in fraction outcomes between students who have $L D, M D$, and students who are not identified.

Ho: There is a significant interaction between student ability group and grade level (e.g., students with LD will be significantly further behind their peers in the sixth grade than in the fourth).

Ho: The influence of each domain-general and domain-specific factor will vary as a function of student ability group and grade level.

Ho: The d2 Test of Attention and results from the cooperation subscale of the SSRS each explain unique amounts of variance in fraction outcomes (i.e., they are not measuring the same thing). 


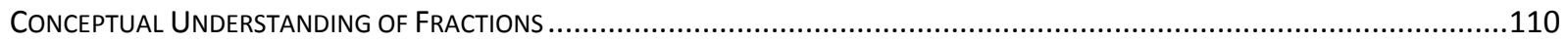

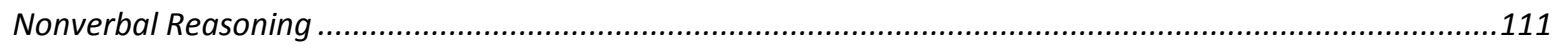

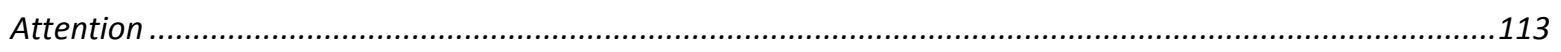

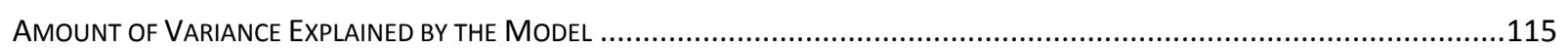

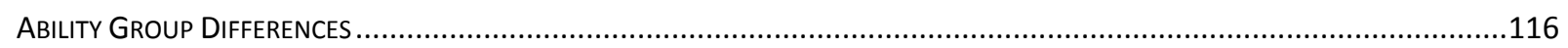

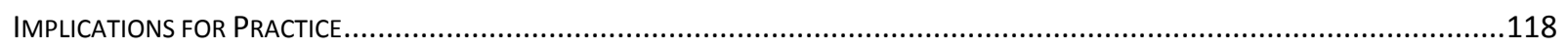

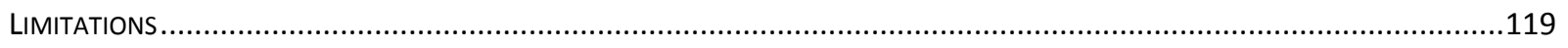

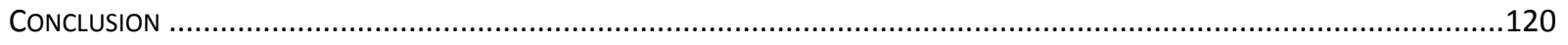

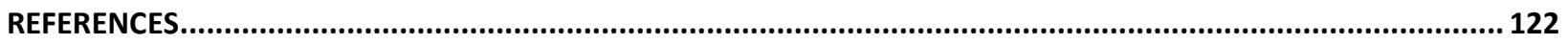

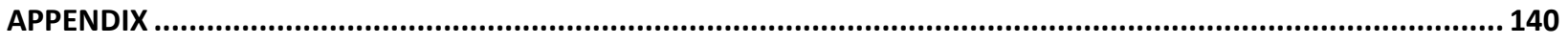

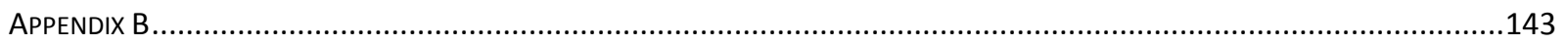




\section{LIST OF FigURES AND TABLES}

Table 1: Study Demographic Information $\quad 57$

Figure 1: Example Item Characteristic Curve $\quad 72$

Figure 2: Proposed Path Analysis Model $\quad 77$

Figure 3: New Path Analysis Model 90

Table 2: Structural Coefficients in the Path Analysis Model 92

Figure 4: Examples of Common Student Fraction Errors 94

Figure 5: Percent of Fraction Difficulties across Grade Level and Skill 97

Figure 6: Fraction Skill Person Ability Estimates by Ability Grouping 101

Figure 7: Grade Level by Ability Group Interaction 102

Figure 8: Ability Group by Attention Interaction Effects on Computation 107

Table 3: Partial and Semi-Partial Attention Correlations 109

Figure 9: Path Analysis Model with Standardized Regression Weights and Covariances 140

Table 4: Means, Standard Deviations, Inter-Item Covariance Matrix and Simple 141 Correlations among Variables

Table 5: DIF Scores: Examining Items for Bias 142 


\section{Fractions Problems}

In recent years educators have become increasingly aware of the importance of developing students' math fluency in a changing 21st century economy (Amit \& Fried, 2002; Kaiser \& Willander, 2005; Kilpatrick, Swaford, \& Findell, 2001). Using the term fluency implies that the acquisition of math skills is not enough in and of itself, rather educational success should be measured by the way in which students utilize their math skills in a thoughtful and meaningful manner. Though fluency has traditionally been associated with reading, math fluency similarly allows a person access to educational, work, and civic opportunities to which a non-fluent person would not have access. For example, in today's highly technical and changing job market a lack of math competence can limit a person's opportunities, and an inability to transfer math skills to an understanding of finances can adversely affect a person's financial stability. A growing body of research demonstrates that students who struggle with math often have worse job success, poor financial decision making, and inhibited social activities (Hibbard, Peters, Dixon, \& Tusler, 2007; McCloskey, 2007; Murnane, Willet, \& Levy, 1995; Parsons \& Bynner, 1997; RiveraBatiz, 1992). Given these outcomes, the goal of math educators should not be limited to teaching mathematical concepts, but it should be expanded to developing students' math fluency which is vital in our increasingly technical economy.

Educators attempting to increase students' overall math fluency must seriously consider the impact of fractions on overall math achievement. Fractions along with decimals unlock the rational number system and allow students greater utility and precision than whole numbers alone. Unfortunately fractions are also one of the most difficult math concepts that students are asked to master in elementary school (Algozzine, O'Shea, Crews, \& Stoddard, 1987; Hecht, Vagi, \& Torgesen, 2007; Riddle \& Rodzwell, 2000; Test \& Ellis, 2005), and a segment of the 
student population continues to struggle with fraction concepts many years after the concepts were initially taught (Calhoon, Emerson, Flores, \& Houchins, 2007; Hecht, 1998; Mazzocco \& Devlin, 2008; National Center for Educational Statistics, 2013). To increase the math fluency of students, researchers need to understand why a segment of the school population continues to struggle with fractions.

\section{Importance of Fractions}

Fractions are important because they are a foundational math component, not merely an individual math skill. Fractions are a way to understand and represent values of numbers that cannot be expressed in the whole number system. This section explores the impact fractions have within the school setting, and then discusses the utility and use of fractions outside of school.

\section{Importance of Fractions Within School}

Fractions are important for student success within school because fraction instruction makes up a significant portion of the elementary school math curriculum and because the fraction skills learned in elementary school are vital to student success in higher-level math classes. The Common Core State Standards (CCSS, 2013) for math reveal that roughly $20 \%$ of all third-grade math standards and $33 \%$ of all fourth- and fifth-grade standards relate directly to fractions. Students who struggle with fractions in elementary school will not only receive poorer grades they can be denied access to higher-level math classes when entering middle school.

Though the number of fraction related standards in middle school is much lower than in elementary school (Virginia Department of Educaiton, 2014), fractions still have a great impact on secondary level math outcomes. Recent research has revealed that fractions, along with division, are the greatest predictors of success in algebra and higher levels of math achievement (Siegler et al., 2012). This is important because algebra is often seen as a "gatekeeper" subject, 
granting access to more advanced coursework and allowing graduation from high school (Smith, 1996). Students who complete algebra successfully have the skills to compete for a wider range of jobs than students who did not complete Algebra.

Though explicit fraction instruction continues in middle school, fraction instruction increasingly turns to using fractions in applied situations (e.g., Standard 6.G.2, where students are asked to find the volume of a prism with a fractional edge; CCSS, 2013). As students move through middle school and into high school they are increasingly expected to be able to utilize fractions in a number of applied ways. Students must understand how to multiply fractions before they can find the volume of a cone in middle school geometry. Students must be able to add, subtract, multiply, or divide fractions to solve equations in Algebra. Other higher-level math classes utilize fractions (e.g., algebra 2, trigonometry, calculus, statistics) and assume that students understand them completely. Students who are not yet fluent with their fraction skills will must devote their mental resources to solving the fraction portions of the problems they are working on, therefore making the acquisition of new math concepts significantly harder (Feldon, 2007; Sweller, 1988; Sweller \& Cooper, 1985). An inadequate master of fractions will hinder a student's ability to progress successfully through higher-level math classes and may negatively affect their chances to graduate from high school.

\section{Utility of Fractions Outside School}

As a former high school teacher working in several different math classes, I often heard students asking “when are we ever going to use this?” Though the life application of what is learned in math class is not always the main point of instruction, I never had difficulty answering when this question was raised regarding fractions. Fractions are ubiquitous in our everyday lives. An understanding of the rational number system is needed when paying taxes, comparing prices, 
understanding discounts, and budgeting. Even outside of monetary matters fractions are commonly used to tell time (e.g., a quarter past three), give medicine to children, follow recipes, and make judgments about the ever-changing world around us.

In addition to the use of fractions in everyday life, there is some evidence to suggest that fractions might be related to success in college and future careers. Currently there are no studies comparing fractions to post-school outcomes, math in general (Hibbard et al., 2007; McCloskey, 2007; Murnane et al., 1995; Parsons \& Bynner, 1997; Rivera-Batiz, 1992) has been directly linked to greater post-school success. As fractions are the greatest predictor of success in high school math classes (Siegler et al., 2012), perhaps future research can explore the relationship between fractions and post school success.

\section{What Students Should Know About Fractions}

Before exploring why some students struggle with fractions, it is important to understand exactly what fraction skills students are expected to have. To determine what most students across the country are expected to know about fractions, all the Common Core State Standards that applied to fractions (CCSS, 2013) were examined. Fraction related standards can be found for early elementary school, for example standard 2.G.3 has students partitioning circles and rectangles into two, three, or four equal pieces. Although initial fraction instruction begins before the third grade, a more intense focus on fraction instruction begins in the third grade.

\section{Grade 3 Standards}

As defined by the CCSS, the focus of third-grade fraction instruction is to develop understanding of fractions as numbers (CCSS, 2013). By the end of the third grade students should be able to place proper fractions (i.e., fractions where the numerator is less than the denominator) on a number line between zero and one. Also, students should understand fraction 
equivalency for common fractions (e.g., 1/2=2/4, 4/6=2/3) and be able to compare the magnitude of two fractions with either common numerators or denominators (e.g., $1 / 3>1 / 4,2 / 5<4 / 5$ ).

\section{Grade 4 Standards}

CCSS for Grade 4 focus on three general areas of student fraction understanding. First, students should extend understanding of fraction equivalence and ordering. Students demonstrate their understanding of fraction equivalence and ordering by being able to model how a fraction $\mathrm{a} / \mathrm{b}$ is equivalent to fraction $(\mathrm{n} \times \mathrm{a}) /(\mathrm{n} \times \mathrm{b})($ e.g., $1 / 3=3 / 9)$ and by being able to compare the relative magnitude of fractions with different numerators and denominators. This skill requires students to be able to create common denominators.

Second, CCSS state that students should be able to build fractions from unit fractions. This means that students should understand that fractions can be pulled apart and put together to create many parts or one whole. For example $3 / 8=1 / 8+1 / 8+1 / 8$; or $3 / 8=1 / 8+2 / 8$. This understanding of fractions allows students to carry out simple operations with fractions. CCSS expect students in the fourth grade to be able to add and subtract mixed numbers and fractions with like denominators (e.g., $21 / 4+2 / 4=23 / 4$ ), and to be able to multiply a whole number by a fraction (e.g., $3 \times 2 / 3=6 / 3)$.

Third, CCSS state that students should understand decimal notation for fractions and be able to compare decimal fractions. Students demonstrating this skill would be able to convert fractions over 10 and 100 into decimals and in turn convert decimals into fractions over 10 or 100 (e.g., $63 / 100=.63$ )

\section{Grade 5 Standards}

CCSS for Grade 5 are broken into two areas of students' fraction understanding. First, students should be able to use equivalent fractions as a strategy to add and subtract fractions with 
unlike denominators. For example, $3 / 4+4 / 5=15 / 20+16 / 20=31 / 20$. In addition by the end of the fifth grade, students should be able to solve word problems that require the addition and subtraction of fractions with unlike denominators.

Second, CCSS for Grade 5 state that students should be able to apply and extend previous understandings of multiplication and division (CCSS, 2013). This understanding of the multiplication and division of fractions is broken into five subcategories. (1) Students should understand that division of two numbers can be expressed as a fraction (e.g., $3 \div 4=3 / 4$ ) and that this understanding can be used to solve real world problems (e.g., how to divide three pies for four people; each person gets 3/4 of a pie). (2) Students should be able to solve multiplication problems involving fractions and be able to model the multiplication of whole numbers by a fraction. (3) Students should understand that fractions can be used to rescale a number (e.g., making a similar rectangle half the size of the original). (4) Students should be able to solve real world problems requiring the multiplication and division of fractions. (5) Students should be able to solve and create models of fraction problems involving division.

\section{Grade 6 Standards}

CCSS for the teaching of fractions continues into the sixth grade where fraction related standards focus is on dividing with fractions (CCSS 6.NS.1), increased understanding of the rational number system (CCSS 6.NS.5-8; although these standards don't mention fractions specifically, fractions are a part of the rational number system), and solving geometric problems with fraction lengths (CCSS 6.G.2). Fractions are definitely a part of the curriculum in the sixth grade, but apart from learning to divide with fractions, the standards are aimed to help students apply and deepen their understanding of previous fraction knowledge.

\section{Grade 7 Standards}


Common Core State Standards continue to call for students to develop and deepen their fraction understanding in the seventh grade. Three standards (CCSS 7.NS.1-3) focus on developing student's ability to use fractions and other rational numbers in applied settings (i.e., $+,-, \mathrm{x}, \div$ ), and three more standards (CCSS 7.RP.1-3) focus on understanding proportional relationships which is an aspect of fractions not focused on much during elementary school.

\section{Grade 8 Standards}

Mention of fractions or rational numbers largely disappears from the 8th grade CCSS. There is one mention of unit rate (CCSS 8.EE.5) but otherwise fraction knowledge is assumed to have already been learned.

\section{SOL Standards}

As an alternative to the CCSS the state of Virginia uses the Standards of Learning. The SOL standards and the CCSS have much in common; the following section will compare and contrast major points of the two sets of standards. Virginia state standards are called the Standards of Learning (SOL), and focus on fraction development begins as early as the first grade (SOL 1.3) where students are asked to identify halves, thirds, and fourths (Virginia Department of Educaiton, 2014). Instruction in the third grade for both sets of standards focuses naming fractions and comparing the relative sizes of fractions with like and unlike denominators. Both sets of fourth grade standards focus on extending students' understandings of the relative value of fractions (e.g., SOL 4.2), and converting fractions to decimals (SOL 4.3d).

One area of difference between the two sets of standards is when multiplication with fractions is introduced. For the CCSS, multiplication is introduced in the fourth grade when students are asked to multiply a whole number by a fraction (CCSS 4.NF.4). The SOL standards don't introduce multiplication until the sixth grade (SOL 6.6). Both sets of standards gradually 
move away from a focus directly on the teaching of fraction skills to using fractions as just another way to represent value when solving practical problems.

\section{Some Students Experience Difficulty Learning Fractions}

In spite of a strong educational focus on fractions many students continue to have difficulty learning fractions. Fraction difficulties arise in elementary school and can persist well into middle school and beyond (Calhoon et al., 2007; Hecht, 1998; Mazzocco \& Devlin, 2008).

\section{Student Difficulties in the Elementary Grades}

Fractions have been called the most difficult concept that elementary students are asked to learn (Algozzine et al., 1987; Hecht et al., 2007). Making fractions especially difficult for elementary students to learn fractions is the fact that elementary teachers often don't know how to explain fractions to their students (Ball, 1990, Tirosh, 2000). Tirosh (2000) found that preservice elementary teachers were able to solve problems involving fractions, yet they were unable to explain the procedures they used when solving the problems. Also, pre-service teachers in Tirosh's study were unaware of the types of errors and misunderstandings students commonly have when working with fractions. Overall Tirosh found that teachers were unprepared to teach fractions to their students.

Whatever the reasons (e.g., poor instruction, difficulty of the material), it is clear that a significant portion of the student population struggle to master different fraction skills. According to results from the most recent National Assessment of Educational Progress (NAEP; National Center for Educational Statistics, 2013) assessment, a little over half of the students tested were able to complete basic fraction tasks successfully. For example, only $54.66 \%$ of students were able to solve $2 / 5+3 / 5+4 / 5$ correctly and only $59.27 \%$ of students were able to answer that a 10-foot string cut into thirds would have larger pieces than if it were cut into 
fourths, fifths, or sixths. Significantly fewer students were able to answer questions requiring a deeper understanding of fractions. For example, only $27.4 \%$ of students were able to answer how many pieces of string a person would have if the person cut $3 / 4$ of a yard into 1/8 of a yard pieces. Only $34.72 \%$ of students were able to correctly answer that a person would not be able to eat half of a pizza and then give away $3 / 8$ of a pizza to two different friends, and only $30.58 \%$ of students were able to correctly identify which fraction in a simple pattern would be the first to have a value greater than one.

\section{Student Difficulties in the Middle School Grades}

As seen by the more applied focus of middle school fraction related standards in both the CCSS and SOL standards, it is evident that students are expected to have a solid understanding of fractions by that point. Unfortunately a significant portion of students struggle with basic fraction concepts years after those concepts were initially taught; for example recent NAEP results showed that $46 \%$ of eighth graders were unable to represent $1 / 3$ on a figure divided into six pieces (National Center for Education Statistics, 2011). Eighth-grade students performed similarly on the one fraction question included in the most recent NAEP assessment; only $41.42 \%$ of students were able to correctly answer a word problem that required them to multiply $1 / 4$ by 18 and then subtract it from a whole number. Individual studies corroborate standardized test findings that many students still struggle to master fractions during their middle and high school years (Calhoon et al., 2007; Hecht, 1998; Mazzocco \& Devlin, 2008).

\section{Difficulties Can be Especially Acute for Students with Disabilities}

Given that such large portions of students struggle to develop fraction fluency, it is not shocking that students with disabilities are especially at risk for failure to master fraction concepts (Calhoon et al., 2007). Though there has been some work focused on the understanding 
of fractions for students with other disabilities (e.g., Hammond, Hirt, \& Hall, 2012; Murphy \& Mazzocco, 2008), learning disabilities (LD) is by far the most common disability and students with LD are very likely to find themselves in academic settings requiring them to work with fractions. Consequently it is important to understand how students with LD learn and utilize fraction knowledge in a different manner than students who have math difficulties (MD) or their non-identified peers. To identify students as having a LD in math, researchers have often relied on a standardized achievement test instead of identification by the local school district. This study used student school-identified disability status and standardized achievement scores to place students in ability groups (MD, LD, typically achieving).

\section{Effect of Disabilities on Math Achievement}

Despite access to adequate learning opportunities, approximately 5\%-13\% of school-age children have cognitive deficits that interfere with their ability to learn math concepts and procedures (Barbaresi, Katusic, Colligan, Weaver, \& Jacobsen, 2005; Geary, 2004). Research has demonstrated that students with disabilities have a high risk of persistently struggling with math (Cawley \& Miller, 1989; Geary, 2004; Judge \& Watson, 2011; Mazzocco, Feigenson, \& Halberda, 2011; Morgan, Farkas, \& Wu, 2011; Toll, Van der Ven, Kroesbergen, \& Van Luit, 2011). These math deficits have been documented early in students' schooling and tend to grow larger as students progress to higher levels of mathematics (Browder et al., 2012). Cawley and Miller (1989) assessed 220 students with disabilities from the ages of 8 through 17. Eight-yearold students with disabilities were found to perform at a first grade level. By the time students were 17 years old their math achievement had only risen to the fifth-grade level. In nine years of schooling students with disabilities made on average four years of growth in math achievement, falling significantly farther behind their peers. 
Perhaps math achievement growth is so slow for students with disabilities because they struggle with a variety of skills necessary for math achievement? Research has revealed that students with disabilities struggle with counting, understanding time, understanding the magnitude of numbers, estimation, solving word problems, and remembering basic math facts (Bryant, Bryant, \& Hammill, 2000; Cawley, Parmar, Lucas-Fusco, Kilian, \& Foley, 2007; Geary, 2004, 2007; Mazzocco et al., 2011). As there are so many components to math, and therefore so many potential areas of deficit, it is difficult to pinpoint the factors that contribute to math difficulties and the effective means of ameliorating those difficulties.

\section{Effects of Disabilities on Fraction Achievement}

Cross sectional and longitudinal studies tracking student fraction performance over time indicate that student understanding of fractions grows as they move to higher grades (Hecht, 1998; Hecht \& Vagi, 2010; Mazzocco \& Devlin, 2008; Seethaler, Fuchs, Star, \& Bryant, 2011; Siegler, Thompson, \& Schneider, 2011), yet there are signs that this growth is not uniform among all students. These studies found that students with MD or LD fraction performance grew at a slower rate than their typically developing peers.

As there is evidence that students with math difficulties are at particular risk of poor fraction performance, it is important to examine how the fraction knowledge of students with MD and LD develops over time. Unfortunately few studies have assessed how the fraction knowledge of students with MDs differs from that of typically developing students. Two studies by Hecht and Vagi $(2010,2012)$ identified students as having MD if they scored in the bottom $25 \%$ of a standardized test. The MD groups performed significantly more poorly than their typically developing peers. Mazzocco and Devlin (2008) examined the differences between students who have MD (10th-25th percentile on a standardized math assessment over two years) 
and students with LD (bottom 10\% over two years). They determined that both groups struggled to order fractions from least to the greatest, but students with LD performed significantly more poorly than students who had MD.

\section{Summary}

Math fluency is linked to educational, career, and economic outcomes and fractions are a significant factor in the development of overall student math fluency. Large portions of the elementary math curriculum focus on the development of student fraction understanding, but significant portions of the overall student population continue to struggle with fractions well into their middle and high school years. Students with MD and LD are more at risk of failing to become fluent in fractions. With such significant amounts of educational time devoted to fractions, why do so many students continue to struggle with fractions? 


\section{Why Do Students Struggle With Fractions?}

Before examining factors that contribute to student fraction deficits, this section will briefly review why fractions are so difficult for students to understand. Fractions don't just introduce new methods of manipulating numbers (e.g., multiplication, division), they are an entirely new way for students to think about numbers. Any operation that can be used to manipulate whole numbers (e.g., add, subtract, multiply, divide, compare) can also be used with fractions. The conceptual shift in the way students view numbers necessitated by the introduction of fractions is often difficult for students to navigate successfully. Before examining the factors that contribute to poor fraction outcomes it is important to explore what makes fractions so difficult for students to understand.

\section{Fractions Introduce the Rational Number System}

Fractions are one of the most difficult mathematical skills students are asked to learn through elementary and middle school (Algozzine et al., 1987; Hecht et al., 2007). Unlike whole numbers, fractions are a decidedly more abstract concept. Students are not able to count fractions on their fingers, and fractions represent numbers that students have never had to consider previously (Wu, 2008). Fractions are difficult because they do not simply represent a new math skill; rather they represent the introduction of the rational number system and with it an entirely new set of procedures for solving problems and new ways of understanding the relative value of numbers.

The whole number system is the way that children are first taught to think about numbers. In this system each number is discrete, for example a student operating under the whole number system would say that there is nothing in between two and three, or sixteen and seventeen. A child could have two or three apples, his or her sister could be sixteen or seventeen years old. In 
a manner this understanding of numbers seems to make sense and is a simple way for children to begin to view the world mathematically. Discrete math has sophisticated uses (e.g., computer programming), but it can be ill-suited to describe the complexity of nature.

Asked the right questions, most any three-and-a-half-year old will testify to the fact that there are important values in this world that cannot be expressed solely through whole numbers. One simply needs to show the child a broken piece of a cookie and an intact cookie while asking questions such as, "Is this a whole cookie?" "Which one is just a part of a cookie?" To address this difficulty mathematicians use the rational number system. A rational number is any number that can be expressed as a fraction, including whole numbers (e.g., 3/1 = 3). The rational number system is continuous rather than discrete and allows single objects to be broken into meaningful parts and still remain part of a whole. For example, an apple cut in half and viewed through the whole number system would be seen as two pieces of apple but the rational number system understands that those two pieces can still represent one whole apple (2/2). Another key component of the understanding of rational number is that there are an infinite number of possible fractions or decimals that can be placed between any two numbers. For example, 13.7, $131 / 2$, and 27/2 all come between the whole numbers 13 and 14. An infinite number of rational numbers can even be placed between two consecutive fractions (e.g., $6 / 8<13 / 16<7 / 8$ ). Rational numbers can be expressed as decimals, but this review and study will focus mainly on the expression of rational numbers as fractions.

From the time students are young they are taught to think about numbers using the whole number system. Students spend years developing beliefs about the magnitude of numbers and how they interact with each other. Students' tendencies to apply whole number approaches to rational numbers has been called whole number bias and is considered one of the major 
impediments to learning to work with fractions properly (Ni \& Zhou, 2005). Whole number bias stems from people's tendency to utilize prior knowledge to understand new material. This system of learning usually works well in mathematics. For example, students learning to multiply can attempt to understand multiplication as repeated addition. The process of integrating new material into what is already known is reinforced throughout students' schooling because it is usually helpful, but in the case of fractions this process actually inhibits the appropriate understanding of rational numbers. Whole number bias is difficult to overcome, because it provides a perceived benefit of efficiency and often results in student inflexibility (Caverni, Fabre, \& Gonzalez, 1990).

Two of the most common fraction misconceptions concern accurately carrying out fraction procedures and understanding the magnitude of fractions. Counting, adding, subtracting, multiplying, and dividing all have a specific set of rules in the whole number system, yet many of these rules do not apply to rational numbers. Students asked to solve 11/12 + 7/9 will often simply add the numerators to obtain one part of the answer and denominators to obtain a second part, thereby obtaining the incorrect answer of 18/21 (Calhoon et al., 2007). Another significant difference between the whole and rational number systems is found in multiplication. When multiplying whole numbers the product is invariably larger than the two multipliers $($ e.g., $7 \times 8=$ 56 and $56>$ both 7 and 8), yet the opposite is true when multiplying fractions with a value of less than one (e.g., $1 / 2 \times 1 / 2=1 / 4$ and $1 / 4<1 / 2)$. Students inappropriately applying their understanding that multiplying always makes numbers larger may have a more difficult time mastering multiplying with fractions.

The second common misconception students have regarding fractions is that of magnitude. In the whole number system, the larger a number is the greater the value of that 
number. By that logic $7 / 15$ would be greater than $3 / 4$ yet this is not true when working with fractions. Students making this mistake are not viewing the fractions as having one value; instead they view each of the numbers in a fraction individually and treat them as if they are whole numbers. Not only does this lead to missed answers when solving fraction problems it demonstrates a misunderstanding of an essential component of fractions. Students learning to work with fractions must learn an entirely new set of conceptual rules and procedures.

\section{Fractions Represent Five Different Constructs}

In addition to difficulties faced trying to understand the rational number system new fraction learners must also understand the various ways that fractions can be conceptualized and used to solve problems. Fractions do not represent one monolithic construct, rather researchers have identified five interrelated fraction sub-constructs: part-whole, ratio, operator, quotient, and measure (Behr, Harel, Post, \& Lesh, 1993; Charalambous \& Pitta-Pantazi, 2007; Kieren, 1976; Pantziara \& Philippou, 2012). Each of these sub-constructs represents a different function of fractions. Some evidence suggests that this theoretical model lines up with the way in which students conceive fractions (Charalambous \& Pitta-Pantazi, 2007), and the five sub-constructs have been widely accepted (Behr et al., 1993; Charalambous \& Pitta-Pantazi, 2007; Kieren, 1976).

Each of the five sub-constructs highlights certain functions and qualities of fractions. Students not understanding one or more of the constructs will have an incomplete conception of fractions. To understand the difficulty students have when attempting to learning about fractions, it is first necessary to explore the five different fraction sub-constructs.

\section{Part-whole Sub-construct}

The part-whole representation of fractions presents an object partitioned into equal 
pieces. Some of the equally divided pieces are selected (e.g., colored or shaded), and the fraction represents a comparison between the number of pieces of the whole selected and the total number of pieces into which the whole is partitioned.

Charalambous and Pitta-Pantazi (2007) highlight several key ideas that are vital to a proper understanding of the part-whole nature of fractions. Students should understand that all parts of a partitioned fraction need to be equal. Students also need to understand that the more pieces a whole is partitioned into, the smaller each piece gets, and that the relationship between part and whole remains the same regardless of whether the parts are rearranged. Also, students need to understand the idea that all partitioned parts are inherently a part of the whole. A student misunderstanding this concept might mistakenly think that $2 / 5$ is a proper representation of $2 / 3$ because he or she did not include the two partitioned pieces in the whole. Lastly, to demonstrate understanding of the part-whole relationship, students must be able to reconstruct (e.g., draw a picture representing 4/5), and repartition (change a whole that was partitioned into thirds into a whole that is partitioned into sixths).

School instruction has leaned heavily towards presenting fractions through the part-whole construct (Wu, 2008). The part-whole relationship is useful for identifying and naming fractions as well as making basic visual comparisons between fractions (Pantziara \& Philippou, 2012; Wu, 2008). Early fraction instruction often presents fractions as pieces of pie. This representation of fractions helps students learn vocabulary and identify fractions but overreliance on pieces of pie to teach the part-whole nature of fractions may actually inhibit students' true understanding of fractions (Wu, 2008). Wu argues that it is awkward to model arithmetic operations with fractions or to model fractions larger than one. For example, what does it mean to multiply two pieces of pie together? These are obstacles that can be overcome or explained, but these difficulties often 
lead teachers and textbooks to concentrate of fractions with values less than one (Wu, 2008), and relying exclusively on fractions that are less than one misrepresents something else important about fractions (namely that they can be used to represent values that are equal to or greater than one).

\section{Ratio Sub-construct}

The ratio sub-construct focuses on the comparative uses of fractions. That is, the numbers in a numerator and denominator of a ratio only have meaning in relation to each other. Key to understanding ratios is the covariance-invariance property (Charalambous \& Pitta-Pantazi, 2007). This property states that the two values of a ratio must change together so that the relationship remains the same. The covariance-invariance property of fractions is what allows us find like denominators without changing the value of the fraction as a whole. The idea of invariance is not found naturally in a simple part-whole understanding of fractions. Further, ratios may be structured as part-to-part (e.g., the ratio of boys to girls in the room is 3 to 5 or 3/5), which is not allowed in the part-whole construct.

According to CCSS standards (2013), students learn about proportions and ratios in the sixth and seventh grades, but some ratio thinking is introduced in earlier years. As mentioned, students learning to find like denominators are being asked to view fractions as having one value determined not by the size of the number in the numerator and denominator, but by the relation between the numerator and denominator.

\section{Operator Sub-construct}

In the operator sub-construct, fractions are viewed as a function that is applied to some object or value (Behr et al., 1993). Fractions acting as operators stretch or shrink an object or number $(3 / 4$ of 4 feet $=3$ feet $)$, or increase or decrease some number of objects $(1 / 3$ of 9 
basketball players $=3$ players). The operator sub-construct of fractions is useful in several everyday life situations (e.g., interpreting the scale of a map, deciding how to divide the check equally at a restaurant). All of the examples given can be solved by the multiplication of fractions. In this way, the operator sub-construct is vital to understanding the multiplication of fractions (Behr et al., 1993). Students should also be able to take two separate operations (e.g., multiplying by 3 and then divide by 4 ) and combine them into one operator (e.g., 3/4).

Multiplying with fractions is generally introduced in the fourth grade and focused on more heavily during the fifth grade (CCSS, 2013). Students must view fractions through the operator construct through almost every higher-level math class that they take (e.g., geometric formulas for area and volume; $Y=4 / 3 X$ - 2 finding the value of $Y$ when given $X)$.

\section{Quotient Sub-construct}

The quotient sub-construct represents fractions being used for division. In this case, fractions are to be understood as one numerical value that is the result dividing two whole numbers. Students being introduced to the quotient sub-construct of fractions are often asked to find "fair shares" of objects (Streefland, 1991). There are two distinctions between a part-whole and a quotient understanding of fractions. First, the part-whole understanding of fractions limits us to one comparison (e.g., a cake divided among 3 friends $=1 / 3$ ), but the quotient nature allows us to introduce another measure into the problem (e.g., 2 cakes divided among 3 friends = 2/3; Charalambous \& Pitta-Pantazi, 2007). Given a problem with two measures, students need to be able to identify the divisor (i.e., numerator) and the dividend (i.e., denominator) correctly. Fractions, when understood using the quotient sub-construct, have no limit to the size of the numerator, which can be smaller, equal to, or larger than the denominator.

\section{Measure Sub-construct}


The measure construct is closely associated with understanding the relative size of fractions and relates strongly to the addition and subtraction of fractions. Number lines are often used to help students understand the measure sub-construct. When viewing fractions on a number line, the denominator tells us how many equal segments are between each unit (e.g., a unit can represent the space between 0 and 1 on a number line), and the numerator tells us how many of these individual segments to count. For example, $9 / 5$ represents counting nine $1 / 5$ segments from a given point.

Though this concept may seem simple at first, students often have great difficulty integrating the concept of fractions as a measure. Lamon (2012) pointed out that because fractions are not a part of the typical counting sequence taught to every child (i.e., 1, 2, 3, 4), students often reject the notion of factions as a single number. This conceptual mistake leads to procedural confusion when adding fractions, for example a student might say that $1 / 2+1 / 3=$ $2 / 5$.

Given the variety of ways in which fractions can be utilized and conceptualized, it is no wonder that so many students struggle to integrate all of the disparate constructs into a common understanding of fractions, and therefore struggle to master fraction skills. To design successful interventions for students struggling with fraction concepts, researchers need a better understanding of how students' fraction skills develop.

\section{Sequence of Learning: Procedural vs. Conceptual}

Both procedural and conceptual understanding is important to students' overall fraction knowledge, but on which should educators first focus teaching? Conceptual understanding of fractions is important because students who lack a conceptual understanding have difficulty transferring their procedural knowledge to new situations (Rittle-Johnson \& Alibli, 1999) or 
being able to explain what it is that they solved through the use of an algorithm (Woodward, Baxter, \& Robinson, 1999). Further, conceptual knowledge helps students choose the correct algorithm to solve problems (Hecht, Close, \& Santisi, 2003). Conversely, there is some evidence that focusing instruction solely on conceptual knowledge does not seem to be an effective method of developing comprehensive fraction skills (Bottge, Rueda, Grant, Stephens, \& Laroque, 2010). Ultimately fully developed proficiency with fractions requires both conceptual and procedural understanding (Pantziara \& Philippou, 2012).

Student acquisition of mathematics is generally viewed by researchers through a procedural-operational or conceptual-structural lens (Pantziara \& Philippou, 2012), and fractions are no different. Students attempting to answer the question $1 / 3+3 / 9$ can either answer that problem by applying an algorithm to find the least common denominator and solving the problem or by realizing that both values are equal to $1 / 3$ and conceptually coming to the conclusion that the answer to the problem is $2 / 3$. Both the procedural and conceptual approach to the problem yield the correct answer, but there is debate among researchers as to which method students should be taught first. One group of researchers has found evidence that many conceptual aspects of fractions are necessary for students to carry out the procedural computation of fraction problems properly (Byrnes \& Wasik, 1991). Conversely other researchers have found evidence that students can solve fraction problems with little conceptual understanding of what they are doing (Hallet, Nunes, \& Bryant, 2010; Hecht \& Vagi, 2012; Kerslake, 1986). Yet a third group of researchers has proposed that conceptual and procedural knowledge of fractions develop together (Rittle-Johnson, Siegler, \& Alibali, 2001).

Two recent studies have examined students' knowledge of procedural and conceptual aspects of fractions in an attempt to explain the contradictory findings about which of these 
concepts develop first (Hallet et al., 2010; Hecht \& Vagi, 2012). If it is true that procedural or conceptual understanding develop in tandem, then cluster analysis of the results should produce a continuous distribution of scores as both skills grow together. If however conceptual knowledge develops first, then the cluster analysis of the results should show a cluster of students with higher conceptual and lower procedural knowledge as well as a continuous distribution of students with their procedural and conceptual abilities in line. According to this view, there should be no groups where students' procedural knowledge exceeds their conceptual knowledge. Conversely, if procedural knowledge were to develop first, then there should be a cluster of students with higher procedural knowledge and low conceptual knowledge, as well as a distribution of students with their procedural and conceptual abilities in line with each other. Hallet et al. (2010) began this line of research with the hypothesis that differences in researchers' findings regarding the development of procedural and conceptual knowledge of factions might be due to individual differences between students reliance on procedural and conceptual knowledge when learning about fractions.

In total, 318 fourth and fifth grade students from the United Kingdom participated in the Hallet et al. (2010) study. Researchers adapted 40 items from the Chelsea Diagnostic Mathematics Tests (Brown, Hart, \& Kuchemann, 1984) and assigned each item to either a procedural or conceptual group. Conceptual items dealt with equivalence, comparison between quantities, or the understanding that fractions can refer to different wholes (e.g., 1/4 of 20 is larger than 1/2 of 6). Procedural problems were identified as problems that could be solved by applying an algorithm without checking for meaning or understanding. Some items like cross multiplication would normally be considered procedural, but the students in the study had not been taught those procedures, therefore those items were classified as conceptual. In all, 9 items 
could not be clearly put in either category, 21 items were considered conceptual, and 10 items were considered procedural. After students took the test, procedural and conceptual scores were regressed onto each other. Hallet and colleagues used residual scores obtained from regression analysis to identify patterns or clusters in student fraction knowledge. Hallet et al. used this procedure as it controls for redundancy between procedural and conceptual knowledge (Cohen, Cohen, West, \& Aiken, 2003). Residuals were scaled with a mean of zero and a standard deviation of one. Positive values represent higher performance than expected when compared to performance on other types of knowledge and negative values represent lower than expected performance.

Hallet and colleagues found five different clusters of student learning patterns when they analyzed the data: (1) lower procedural (average conceptual); (2) lower conceptual (average procedural); (3) higher conceptual-lower procedural; (4) higher procedural-lower conceptual; and (5) higher (high on both procedural and conceptual items). Students in the high conceptual clusters demonstrated better accuracy on fraction computation tasks than students with lowerthan-expected conceptual knowledge. As discussed previously, theories about fraction development predict specific outcomes. For example, if student conceptual understanding develops first then results of Hallet et al. should show a group of students with higher conceptual-lower procedural understanding and a distribution of students whose procedural and conceptual understanding are developing in tandem. Results from Hallet et al. did not fit the patterns predicted by the three theories of fraction development (i.e., procedural-first, conceptual-first, develop in tandem). These results indicate that there are individual differences between students that determine the manner in which their fraction knowledge develops. If this is true, then more research needs to be done examining what factors influence which cluster of 
fraction learning students will most closely resemble. For example, it has been demonstrated that student attention issues not only affect the fraction achievement of students with MD, attention issues explain most of the difference in fraction knowledge growth between students with and without MD (Hecht \& Vagi, 2010). Perhaps individual student deficits (e.g., attention, nonverbal reasoning, working memory) explain the different performance patterns found by Hallet et al.

Hecht and Vagi (2012) performed a similar study examining individual patterns of strengths and weaknesses in students' procedural and conceptual understanding of fractions. Hecht and Vagi adopted Hallet's procedures for identifying items in assessments as procedural or conceptual, though they did not consider whether students had been taught specific concepts. They followed 181 students from fourth to fifth grade, allowing the researchers to examine the stability of clusters over time in addition to examining the relationship between the development of procedural and conceptual knowledge of fractions. Participating students completed 24 fraction computation problems focused only on the addition and multiplication of fractions and mixed numbers. Participants also completed a battery of conceptual fraction tasks assessing their ability to convert pictures to symbols (13 items), symbols to pictures (18 items), compare fraction size (24 items), solve fraction addition problems presented in pictorial form (13 items), solve word problems (18 items), and estimate sums of fractions (12 items). Hecht and Vagi also assessed student intelligence using the Wechsler Abbreviated Scales of Intelligence (WASI; Wechsler, 1999). A principal component factor analysis across all hypothesized conceptual items yielded only one factor and a confirmatory factor analysis showed that one factor was a good fit for the data in both the fourth and fifth grades. Hecht and Vagi created a single composite conceptual knowledge score for each student. They used the same procedures utilized by Hallet et al. (2010) to analyze the data. 
Hecht and Vagi (2012) identified four unique clusters of students in the fourth grade and seven unique clusters for the fifth grade. The four clusters reported in the fourth grade replicated four of the five clusters found by Hallet et al. (2010; i.e., lower conceptual (average procedural), higher conceptual-lower procedural, higher procedural-lower conceptual, and higher (both procedural and conceptual). The one cluster not replicated from Hallet et al. (2010) was the lower procedural (average conceptual) cluster. Results for the fifth grade were decidedly more complex. Of the seven clusters, one showed lower procedural (average conceptual) understanding, two clusters showed lower procedural-higher conceptual, one cluster was higher in both procedural and conceptual, and finally three clusters revealed lower than expected conceptual-higher procedural. These clusters differed in the magnitude of differences between expected procedural and conceptual understanding but again replicated four of the five clusters described by Hallet et al. (2010). For fifth-grade students the one cluster that was not replicated was the lower conceptual (average procedural) cluster. Examination of the percentage of students exhibiting high procedural and low conceptual (27\%), low procedural and high conceptual (34\%), and relatively high on both (39\%) reveal that roughly one third of students fall into each category. Subsequent analysis revealed that cluster membership was not highly stable, though students who initially had high procedural and low conceptual knowledge were most likely to remain in a similar cluster in fifth grade.

As examination of the results have all been relative to expected performance up to this point (e.g., "high conceptual" means the student showed better than expected conceptual understanding based upon some gauge of intelligence or ability, not in relation to other students), Hecht and Vagi (2012) examined the overall accuracy of the different clusters, not just in relation to expected performance. They found that high levels of procedural and conceptual knowledge 
can both increase accuracy in the absence of the other though conceptual knowledge seemed to have a greater overall impact on accuracy than procedural knowledge did.

Hecht and Vagi's (2012) study included 55 students with MD. Students falling in the bottom $25 \%$ on the Woodcock-Johnson III Calculation composite (WJIII: Woodcock, McGrew, \& Mather, 2001) were identified as having MD. A chi-square analysis of cluster membership indicated that there were significantly more students than expected with MD in the low concept cluster, and there were significantly fewer than expected in the high concept or high procedures cluster. Similarly in the fifth grade there were significantly more students with MD in the low procedures group than expected, and there were significantly fewer students with MD in the high concepts and high procedures clusters.

These two studies (Hallet et al., 2010; Hecht \& Vagi, 2012) provide evidence that students' initial understanding of fractions can develop differently but little is known as to why this is so. Researchers need to understand what factors influence the differential development of fraction learning found between students of all ability types. Given their difficulties with mastery of fractions, the learning characteristics of students with learning disabilities are of particular interest.

\section{Students’ with Disabilities Learning Characteristics}

Students with disabilities are known to have learning characteristics that might interfere with their acquisition of fractions knowledge. Understanding what factors contribute to poor student outcomes for students with LD has the potential to help guide intervention and identify students at risk of failure before they fail. Some common deficits identified in students with LD are self-monitoring, working memory, executive function, and phonological processing (Watson \& Gable, 2012). Although these examples of deficits only cover a small portion of possible 
explanations for failure by students with LD, closer examination of one of these cognitive factors can demonstrate how these factors potentially influence student achievement.

Working memory is a processing resource with limited storage capacity. Students solving problems simultaneously hold and manipulate information in their working memory (Baddeley, 2000). Theoretically any student who has a lower working memory capacity would find it difficult to retain information, filter out irrelevant information, synthesize information, and demonstrate flexibility while thinking. For example, if a student had to find out how much money she would have after paying for a $\$ 25$ shirt that is on sale for $25 \%$ off when she originally had $\$ 70$, so much attention could be focused on how much she paid for the shirt that she forgets to subtract the result from her total. Alternatively the student could have difficulty figuring out that she should actually be looking for $75 \%$ of the $\$ 25$ shirt. Students with working memory issues have difficulty managing the competing facts and procedures that need to be done to successfully complete a problem. Research has generally supported the view that working memory is a strong predictor of overall mathematics achievement (e.g., Alloway \& Passolunghi, 2011; Andersson \& Lyxell, 2007; Fuchs et al., 2006; Swanson \& Beebee-Frankenberger, 2004). As working memory has the potential to disrupt student acquisition of fraction knowledge, perhaps differences in working memory or other similar cognitive factors can explain the different learning patterns found by Hallet et al. (2010) and Hecht an Vagi (2012).

\section{Math Performance of Students with Disabilities}

As students with LD have cognitive deficits, it is important to identify how these deficits affect students' mathematics performance, i.e., where are students with disabilities falling behind in mathematics? Students with LD have several different math specific deficits that researchers have identified. Students with LD often have difficulty understanding the number system and 
number concepts; understanding abstract concepts of time, temperature, directions, and speed; difficulty counting; remembering computation facts; understanding place value, estimating; and solving word problems among other deficits (Bryant et al., 2000; Cawley et al., 2007; Geary, 2004, 2007; Gilmore, McCarthy, \& Spelke, 2010; Watson \& Gable, 2012).

Whatever the factors influencing students with LD are, it is clear that the results are low achievement. The Nation's Report Card reported that 25\% of students without disabilities compared to $45 \%$ of students with LD completed a below-standard curriculum (National Center for Education Statistics, 2011). The National Longitudinal Transition Study-2 (NLTS-2; Wagner, Newman, Cameto, Levine, \& Garza, 2006) showed that more than $75 \%$ of students with disabilities are achieving below the mean. Calhoon et al., (2007) found that high school students with LD were fluent in computation skills at the second and third grade levels. Participants in that study had difficulty subtracting numbers with multiple digits, regrouping, multiplying, dividing, and working with rational numbers. Many other researchers have corroborated the fact that students with LD have an acute risk of mathematics failure (Cawley \& Miller, 1989; Geary, 2004; Judge \& Watson, 2011; Mazzocco \& Devlin, 2008; Morgan et al., 2011; Mundia, 2010; Toll et al., 2011).

Given that students with disabilities struggle with general math achievement and that fractions are difficult for all students it is reasonable to assume that students with LD are particularly at risk for failing to master necessary fraction skills.

\section{Fraction Performance of Students with Disabilities}

Though the research base describing fraction deficits for students with LD is still emerging, initial evidence indicates that disabilities put students at a high risk for fraction failure. There are only a few descriptive studies of fraction performance that included students with 
disabilities. In one study of 224 high school students with LD, students only attempted $9 \%$ and $8 \%$ of fifth and sixth grade level fraction problems while their non-identified peers attempted problems at a much higher rate (Calhoon et al., 2007). Another study indicated that students with LD have less well-developed conceptual understanding of proportional reasoning and less welldeveloped strategies to solve problems than typically developing students (Grobecker, 1999). Mazzocco and Devlin (2008) studied LD, MD, and typically achieving students' abilities to identify equivalent pairs of fractions and found that students with LD fared significantly worse than the other two groups.

A slightly larger group of intervention studies have attempted to improve various fraction skills of students with LD. Studies have found cue cards (Joseph \& Hunter, 2001), mnemonic devices (Test \& Ellis, 2005), and schema based instruction (Xin, Jitendra, \& Deatline-Buchman, 2005) to be effective interventions for students with LD. Several other studies (Bottge, 1999; Bottge \& Hasselbring, 1993; Bottge, Heinrichs, Mehta, \& Hung, 2002; Bottge et al., 2010) have focused on using contextualized or anchored instruction to improve students' problem solving skills. Results of this line of research seem to show the most promise when the contextualized instruction is paired with extra instruction (e.g., Mastery Fractions videodisc program). Another line of research focused on the effectiveness of the Mastery Fractions videodisc program (Kelly, Carnine, Gersten, \& Grossen, 1986; Miller \& Cooke, 1989; Moore \& Carnine, 1989; Woodward \& Gersten 1992) and generally found it to be more effective than basal math curriculum. Finally two studies examined the effectiveness of presenting fractions in the concrete, representational, abstract (CRA) sequence of instruction (Butler, Miller, Crehan, Babbitt, \& Pierce, 2003; Jordan, Miller, \& Mercer, 1999). Both studies found CRA instruction to be effective for most students but Jordan et al., found that students with LD performed no better with CRA instruction than 
they did with basal mathematics instruction.

Considering the importance of fractions in students' mathematics development it is surprising that there are only 20 (according to a literature review the main author completed) studies that examine how students with LD perform on fraction tasks. Four more studies have been conducted but have only examined students with MD instead of students with LD (i.e., Flores \& Kaylor, 2007; Kolikant \& Broza, 2010; Hecht \& Vagi, 2010, 2012). Part of this is due to the logistical difficulty in finding large enough samples of students with math LD to analyze statistically. In fact several of the studies mentioned in the previous paragraphs included students with LD but did not disaggregate their findings from students who simply had MD (e.g., Bottge \& Hasselbring, 1993; Kelly et al. 1986; Moore \& Carnine, 1989). Although the common use of MD in math research does provide some data about the performance of students who are most at risk, the practice cannot capture the differences that do exist between students who simply have MD and students who have LD (e.g., Mazzocco \& Devlin, 2008).

\section{Math Difficulties and Learning Disabilities}

By simply comparing students whom they identify as having MD with non-disabled students rather than comparing $\mathrm{LD}, \mathrm{MD}$, and non-disabled students, researchers are aggregating students with very different abilities together into their MD group. This is a rather typical practice in the education literature. Using the term MD to examine struggling learners has the benefit of allowing larger groups of participants (thereby, increasing statistical power, for example) than simply sticking to students with identified LD.

Cutoffs for MD groups differ from study to study but often hover between the bottom 2033\% of students (e.g., Fuchs et al., 2010; Hecht \& Vagi 2010; Powell, Fuchs, Fuchs, Cirino, \& Fletcher, 2009). Unfortunately these groups are often more fluid than they are fixed. For 
example, Mazzocco and Devlin (2008) identified 147 potential participants for their longitudinal study but eventually had to eliminate 36 participants (24\%) because they fell into the MD (11th25th percentile) or LD (0-10th percentile) group one year but not another. Similarly Hecht and Vagi (2010) originally identified 55 students as having math difficulties (bottom 25th percentile) but $17(31 \%)$ of those students no longer met the qualifications for having math difficulties the next year.

The trouble with relying solely on MD to research and explore the learning profiles of struggling students is that those who have LD are lumped in with a larger group. The prevalence rate of LD is around 5\% of the total school population (U.S. Department of Education, 2012). Consequently students with LD participating in a study that is examining struggling learners are probably going to be outnumbered five to one. As Mazzocco and Devlin (2008) found differences between students in their MD and LD groups on the conceptual task of ordering fractions, perhaps there are further differences between these groups that are not being identified due to current research practices? In addition to simply identifying performance differences between students with LD, with MD, or their typically developing peers researchers need to examine what cognitive factors contribute to the differences found between groups.

\section{Examining Sources of Fraction Learning Differences}

Fraction skills are a major difficulty for students moving from the whole number system and basic arithmetic to more advanced math, such as algebra and geometry (Hecht et al., 2003; Kilpatrick et al., 2001). This transition is especially difficult for students with MD or LD. For researchers to address student fraction deficits more accurately, it is important to understand what factors contribute to student difficulty developing fraction skills. Hecht (1998) argued that a complete theory of emerging individual differences in fraction skills must take into account the 
cognitive and behavioral characteristics of students that limit the acquisition of new types of math knowledge. For example, if a student has difficulty with working memory, then that cognitive characteristic might inhibit his or her ability to develop fraction skills adequately. Behavioral factors like students' attention can limit the amount of time that students are able to engage in academic activity and therefore limit their acquisition of new fraction skills. Before discussing research on the individual factors that contribute to math performance, we will discuss two different types of factors that influence fraction outcomes.

\section{Domain General and Domain Specific Predictors}

Though all fractions problems involve some sort of operation with rational numbers different types of fraction problems require different abilities or skills to complete. For example, the ability to read may not have much to do with solving $1 / 2+1 / 3$, but would heavily influence a student's ability to solve a word problem involving the same two fractions. Likewise a student's fluency with the multiplication of whole numbers can influence his or her ability to solve fraction multiplication problems but not affect their performance on fraction addition problems. Researchers working to find what factors influence student mathematics achievement have adopted the framework of domain-general and domain-specific to describe two different types of factors that predict student mathematics growth (Hecht et al., 2003; Hecht \& Vagi, 2010; Seethaler et al., 2011).

Domain-specific attributes are factors which influence students' ability to solve specific types of problems but might have little influence on student success for other problem types (Hecht \& Vagi, 2010; Kail, 2004). Two common domain-specific deficits are reliance on error prone counting based strategies instead of fact retrieval (Geary, 1993; Jordan \& Hanich, 2003; Mabbot \& Bisanz, 2008), and poor conceptual knowledge (e.g., commutativity, inversion, order 
irrelevance when counting objects; Geary, 1994; Jordan \& Hanich, 2003). These deficits limit student capacity to solve specific types of problems (i.e., mathematics problems) but would have little influence on literacy or social studies.

There is also evidence of domain-general factors influence on mathematics achievement. Domain-general attributes are factors that influence students' ability but are not specific to a particular mathematical skill. Working memory, attention, processing speed, and nonverbal reasoning are just some examples of domain-general cognitive factors. Each of these deficits could be expected to inhibit fraction knowledge but could reasonably influence other academic areas as well (e.g., literacy, science, social studies).

\section{Predictors of Mathematics Performance}

Previous research in arithmetic and mathematics in general and fractions specifically has examined a diverse array of factors as potential predictors of outcomes. Using both prospective and cross-sectional methods, researchers studying general mathematics outcomes have linked processing speed (Fuchs et al., 2006, Fuchs et al., 2008; Geary, Hoard, Byrd-Craven, Nugent, \& Numtee, 2007) and working memory (Seethaler et al., 2011) to whole-number computation outcomes. Although working memory has been shown to influence calculation and wordproblem skill (Fuchs et al., 2006; Swanson, 2006; Swanson \& Beebe-Frankenburger, 2004), many cognitive factors have only been linked to word problems success. Concept formation, nonverbal reasoning, sight word efficiency, language skill, and reading have all been associated with word problem success (Fuchs et al., 2006; Swanson, 2006; Swanson \& BeebeFrankenburger, 2004). The differential effects of various cognitive factors on different mathematics tasks lends support to the view that individual types of problems within the wholenumber mathematics domain each require a different set of skills. 


\section{Predictors of Fraction Performance}

With more specific regard to fractions learning a recent line of research has sought to identify domain-general and domain-specific cognitive and behavioral factors that influence fraction achievement. Hecht (1998) began this line of research by examining the influence of students' conceptual and procedural knowledge of fractions as well as students' arithmetic fluency. Hecht tested 103 seventh and eighth grade students in a cross-sectional design. Participants conceptual understanding (i.e., understanding the part-whole and measurement interpretations of fractions), procedural understanding (i.e., the specific steps needed to solve fraction problems), and arithmetic fluency (i.e., the speed that a student solved single digit multiplication and addition problems) were assessed along with three fraction outcome measures (i.e., fraction calculation, fraction word problems, and fraction estimation). Hecht found that conceptual and procedural understandings were both significant predictors of fraction computation and word problem solving although conceptual knowledge was the only unique predictor of fraction estimation success after accounting for other variables (e.g., grade level of participant, word level reading, and vocabulary scores). Surprisingly arithmetic fluency was not a significant predictor of any of the three fraction outcomes. Hecht noted that only $5 \%$ of the errors participants made were due to calculation mistakes and the vast majority of errors were due to procedural mistakes. This discrepancy in error rates indicates that simple arithmetic instruction will have little chance of affecting fraction calculation performance.

Hecht et al. (2003) extended on Hecht's previous study by assessing 105 fifth grade students and by adding two domain-general factors in addition to the domain-specific conceptual knowledge and arithmetic fluency factors assessed in the previous study. Hecht et al. (2003) used path analysis to create a model whereby they examined whether the domain-general factors had a 
direct affect on fraction outcomes or whether their influence was mediated by domain-specific factors. As arithmetic speed alone did not seem to be an effective predictor of fraction outcomes in his previous study Hecht et al. (2003) added measures of accuracy and strategy students used to solve problems to the arithmetic fluency measure. Analysis of the data revealed that conceptual knowledge was an independent predictor of all three fraction outcomes (i.e., computation, word problems, and estimation) even after controlling for other predictors (i.e., working memory, attention, and word level reading) and arithmetic fluency was only a unique predictor of fraction computation. Working memory was significantly related to fraction computation and word problem solving though the relation to fraction computation was significantly reduced after accounting for arithmetic fluency. These results demonstrate that the influence working memory has on fraction computation is mediated by arithmetic fluency. Attention, or more specifically on-task behavior, was significantly related to all three fraction outcomes but that influence was largely mediated by conceptual knowledge and arithmetic fluency. These results indicate that some but not all of the influence that domain general factors have on fraction outcomes is mediated by the domain specific mathematics factors.

Hecht and Vagi (2010) expanded this line of research by conducting a two year longitudinal study of 181 fourth and fifth grade students, including 55 students with math disabilities. They used the same domain-general and domain-specific factors as Hecht et al., (2003). As this was a longitudinal study Hecht and Vagi examined the affects that attention, working memory, conceptual knowledge, and arithmetic fluency had on students' growth on fraction computation, estimation, and word problem solving. When not separating participants into ability based groups working memory, attention, conceptual knowledge, and arithmetic fluency all had a significant effect on fraction outcomes. Group differences between participants 
with MD and their non-identified peers revealed that students with MD consistently performed below their peers and showed less growth between fourth and fifth grade. Differences in fraction skills between the two groups were mediated by students' conceptual knowledge of fractions and their attentive behavior. In fact, group differences in fraction computation and word problem performance were completely explained by attention and conceptual knowledge. Working memory and arithmetic fluency did not account for differences in fraction performance between groups. These results indicate that though working memory and arithmetic fluency can affect fraction achievement they are not the deficits that explain students with MD's poor performance on fraction skills. Thus interventions designed to target working memory and arithmetic fluency for students with MD may have little influence on fraction performance.

Siegler et al. (2011) conducted a study in which they focused on participants' understanding of fraction magnitudes. Siegler et al. compared 24 sixth- and 24 eighth-grade students' performances on fraction computation problems (e.g., $3 / 5+1 / 2,3 / 5-1 / 2,3 / 5 \times 1 / 2$ ) and a standardized math achievement test (i.e., Pennsylvania System of School Assessment fifthand seventh-grade math tests) to their ability to place fractions on number lines and compare the sizes of fractions accurately. They found that knowledge of fraction magnitudes vary greatly from individual to individual and that arithmetic proficiency and general math achievement scores are strongly correlated with a command of fraction magnitude. Another key finding of this study is that students continue to develop an understanding of fraction magnitude at least through eighth grade despite not having any formal fraction instruction. Although this study did not directly test cognitive factors influence on fraction achievement it does provide some evidence that previous math skill is linked in some way to student understanding of the value of fractions.

Seethaler et al. (2011) conducted a longitudinal study with 688 participants tested in both 
the third and fifth grades. They extended the line of research started by Hecht and colleagues by assessing third grade students and introducing processing speed, nonverbal reasoning, concept formation, and alternative language measures as possible predictors of fraction skill growth. They investigated which cognitive factors uniquely accounted for development of computational skill with rational and whole numbers while controlling for incoming calculation skill. Results indicated that whole number calculation skills and rational number calculation skills are influenced by many of the same cognitive factors: incoming whole-number calculation skill, concept formation, nonverbal reasoning, and working memory (numerical executive control). Language was a unique predictor of rational number calculation but not of whole number calculation. This study provided the first evidence of nonverbal reasoning being linked to whole or rational number calculation, though previous studies have linked nonverbal reasoning to solving whole number word problems (e.g., Fuchs et al., 2006; Fuchs et al., 2010).

\section{Cognitive Deficits for Students with Disabilities}

Researchers examining the sources of deficits for students with disabilities have identified several cognitive factors that affect mathematics achievement. Visual or spatial deficits (e.g., Bull, Espy, \& Wiebe, 2008; Hanich, Jordan, Kaplan, \& Dick, 2001; Zorzi, Priftis, \& Umilta 2002), self monitoring (e.g., Ivrendi, 2011), executive function (Andersson, 2008; Blair \& Razza, 2007; Bull \& Scerif, 2001), and phonological processing (DeSmedt \& Boets, 2010; Murphy, Mazzocco, Hanich, \& Early, 2007; Vukovic \& Siegel, 2010) are just some of the cognitive deficits that have been identified as contributing to the poor math performance of students with LD. Watson and Gable (2012) state that the most commonly suggested cognitive deficit responsible for poor mathematics performance is working memory (e.g., Alloway \& Passolunghi, 2011; Berg \& Hutchinson, 2010; Bull et al., 2008; Holmes \& Adams, 2006; Kyttala 
\& Lehto, 2008; Mabbott \& Bisanz, 2008; Meyer, Salimpoor, Wu, Geary, \& Menon, 2010; Murphy et al., 2007; Passolunghi \& Siegel, 2004; Passolunghi, Vercelloni, \& Schadee, 2007; Rasmussen \& Bisanz, 2005; Swanson, Jerman, \& Zheng, 2009; Swanson \& Sachse-Lee, 2001; Tronsky, 2005). In fact, working memory has been shown to be significantly linked to fraction performance (Hecht et al., 2003, Seethaler et al., 2011). Considering all the evidence for the affect working memory has on the math achievement of students with LD and the fact that working memory has been linked to fraction performance it would not be shocking to find that working memory is a significant reason students with LD struggle with fractions. Surprisingly Hecht and Vagi (2010) found that working memory did not account for any significant difference in growth of fraction performance between students with MD and typically developing students. Due to findings like this it is important for future research to more thoroughly investigate the interaction between student ability and cognitive and behavioral factors influencing fraction performance.

\section{Summary of the Sources of Fraction Learning Differences}

Researchers have identified several cognitive and behavioral factors that affect fraction performance (i.e., attention, working memory, arithmetic fluency, conceptual understanding of fractions, nonverbal reasoning, processing speed, concept formation, language) but little is known about the affects of each of these factors and the mechanism by which each factor influences fraction performance. Many of these factors (i.e., nonverbal reasoning, processing speed, concept formation, language) have only been linked to fraction computation and were only assessed in one study. To develop a fuller understanding of how these cognitive factors contribute to poor fraction outcomes more research needs to be conducted.

Nonverbal reasoning has previously been linked to whole number word problem solving 
(Fuchs et al., 2006) but not whole number calculation. Therefore it was interesting that nonverbal reasoning was significantly related to fraction computation. This study focused on the influence that nonverbal reasoning has on fraction outcomes to determine (1) does nonverbal reasoning influence fraction word problem solving as it does with whole number word problem solving, and (2) whether the significant relationship between nonverbal reasoning and fraction computation can be replicated as nonverbal reasoning has not been related to whole number computation.

Attention is not only significantly related to fraction outcomes (i.e., calculation, word problem solving, estimation), it is significantly related to arithmetic fluency and the conceptual understanding of fraction (Hecht et al., 2003, Hecht \& Vagi, 2010). Neuroscientists have described three different aspects of attention, alerting, orienting, and executive control (Fan, McCandliss, Sommer, Raz, \& Posner, 2002; Keehn, Lincoln, Muller, \& Townsend, 2010; Posner \& Fan, 2008; Posner \& Petersen, 1990). To date the only measure of attention's relation to fractions has come through teacher observation of on-task behavior (Hecht et al., 2003; Hecht \& Vagi, 2010). On-task behavior can serve as a global estimate for attention, but what specific aspects of attention are responsible for attention's influence on fraction achievement?

\section{Attention}

Presumably children who are able to attend to classroom activities and instructions should acquire stronger math skills than students who have difficulty attending. Research has shown that attentive behaviors in the classroom are linked with variance in the gaining of academic skill (Bennett, Gottesman, Rock, \& Cerullo, 1993; McKinney \& Speece, 1986; Wentzel, 1991). For example, Chen, Rubin, and Li (1997) found a correlation of .52 for middle school students between variability in an achievement test and classroom behavior. Cirino, 
Fletcher, Ewing-Cobbs, Barnes, and Fuchs (2007) found evidence that attention issues significantly inhibited the math performance of 291 third and fourth grade students. Indeed many other researchers have highlighted the connection between attention issues and math performance (e.g., Barkley, Fischer, Edelbrock, \& Smallish, 1990; Brown \& Borden, 1986; Hecht et al., 2003).

Zentall (1993) argues that students with attention issues don't have deficits so much as they have an attention bias. It is not that students with attention issues don't pay attention; it is that they often pay attention to the wrong things. Student with attention issues are more likely than their peers to be distracted by changes in the environment around them (e.g., color changes, movement; Copeland \& Wisniewski, 1981). A second issue common for students with attention issues is an inability to maintain focus on a task when there are decreasing levels of novelty (Zentall, 1993). Zentall points out that the inability to maintain focus on decreasingly novel tasks can cause difficulty for students trying to develop rote skills (e.g., arithmetic facts, multiplication facts).

\section{Attention as a Predictor of Fraction Performance}

Of all the cognitive and behavioral factors that have been shown to influence fraction outcomes no other factors' influence has been as pervasive as attention. Hecht et al. (2003) found that attention was a significant predictor of both domain-specific factors (i.e., conceptual understanding, arithmetic fluency) and all three fraction outcomes (i.e., computation, word problems, estimation), though the relationship between attention and fraction computation and estimation was not significant after controlling for the influence of domain-specific factors. Although the effect of attention on fraction outcomes was mitigated by other factors in the Hecht et al. (2003) study a subsequent study found that attention uniquely predicted growth on all three 
fraction outcomes (Hecht \& Vagi, 2010). Also, Hecht and Vagi tested the differential effects of attention on the growth of student fraction performance over time and found that attention along with conceptual understanding explained $100 \%$ of the variance found between students with and without MD.

\section{Theory}

As researchers in the education field it can be difficult to connect the mental level of description of behaviors (e.g., attention, on-task) with the cognitive processes going on at the anatomical level. Developments in brain imaging technology have led to a line of research that has slowly attempted to bridge the gap between neuroscience and observable behavior (Posner \& Fan, 2008). These studies generally focus on which areas of the brain are activated when participants engage in certain activates. These studies have identified several networks that contribute to a person's ability to focus on specific tasks or stimuli. Posner and Fan (2008) likened these different networks of the brain to an organ system, one in which each part of the network plays its own individual role but attention is only reached when all parts work together. Researchers have commonly broken the attention related networks into three distinct groups based largely on the function of each network: alerting, orienting, and executive control (Fan et al., 2002; Keehn et al., 2010; Posner \& Fan, 2008; Posner \& Petersen, 1990).

Alerting. Alerting attention refers to a person's ability to maintain a state of readiness and expectancy (Mezzacappa, 2004). Researchers have found that infants develop the capacity for alerting by three months of age (Mezzacappa, 2004) and that the ability to alert develops slowly over time often extending into young adulthood (Rueda et al., 2004). Visual alerting is the ability to shift one's focus to a particular stimulus. In several neuroscience alerting studies participants have been asked to focus on a specific target area and then researchers present a 
stimulus. Researchers record how quickly participants react to the stimulus compared to how quickly they react when not cued beforehand. Participants without attention issues generally respond more quickly to the stimulus when they have been cued to focus on a target area and those with attention issues show little or no improvement in response time (Posner \& Fan, 2008). These results indicate that students with attention issues have a limited capacity to anticipate or prepare to receive information even if they know it is coming.

Orienting. Visual orienting is the ability to disengage attention from one target to focus on a new stimulus. In several neuroscience orienting studies participants have been asked to focus on a specific target area and then researchers present a stimulus outside of the cued area. Participants with orienting attention issues have difficulties linked to specific areas of the brain. For example, participants with damage to the posterior lobe benefit from receiving a cue for where to target their attention but often have difficulty disengaging their attention from the targeted area when the stimulus falls outside of the cued area (Posner \& Fan, 2008). Lesions to the thalamus cause people to not be able to effectively engage their attention even when given a prior cue and with the stimulus presented in the targeted area (Posner \& Fan, 2008). There is also evidence that the right and left hemispheres of the brain have an influence on attention. The right hemisphere of the brain is biased towards processing big picture and the left hemisphere is biased towards processing the small details (Robertson \& Delis, 1986). Researcher figured this out by presenting participants with brain lesions on one side of the brain or the other with large letters constructed out of smaller versions of the same letters. Patients with damage to the right hemisphere copied the small letters but not realize that the small letters join together to form a large letter; the opposite is true of participants with left brain damage.

Research has shown that different parts of the brain influence people's ability to orient 
towards new stimuli. Each part of the orienting attention network contributes to a person's ability to selectively pay attention to what is important, but what does orienting look like in the classroom? Hallahan and colleagues did a series of studies on what they called selective attention (e.g., Hallahan, Gajar, Cohen, \& Tarver, 1978; Hallahan, Kauffman, \& Ball, 1974; Tarver, Hallahan, \& Cohen, 1977) but would now fall into the orienting category of attention. Hallahan et al., (1978) used a modified version of Hagen's task (1967) to assess students' ability to orient. In this task researchers presented 18 posters each with seven paired pictures. Each pair of pictures had one household item and one animal (e.g., table-dog, clock-deer) in random order. Researchers presented each poster to the participants for 12 seconds and participants were asked to remember the order in which they see the animals on the poster. The efficiency with which participants remembered the order of the animals determined their selective attention score. Immediately after the recall portion of the test students were asked to pair the animals with the corresponding household items they were not told to focus on. Student ability to remember these household item animal pairings was labeled incidental recall. They found that students' ability to orient to important or central information increases developmentally and that students with LD commonly have deficiencies in their ability to orient efficiently. Paired with a deficient ability to orient to important or targeted information was the result that students with LD were just as likely to remember incidental or non-targeted information.

Executive Control. Executive control is most needed in situations involving decisionmaking, error detection, planning, novel responses, and overcoming habitual actions (Posner \& Fan, 2008). Essentially executive control relates to the brain's ability to resolve conflicts between competing areas of the brain (Botwinick, Braver, Barch, Carter, \& Cohen, 2001). A common method of assessing executive control is through the Stroop test (Posner \& Fan, 2008). 
The Stroop test presents the participant with extraneous stimuli that can inhibit the participant's ability to respond accurately to a prompt. For example, the participant might be asked to read the word blue, but the word blue is printed in red ink. The presentation of the word blue in red can lengthen the amount of time it takes participants to respond accurately. Conversely participants might be asked to name the color that the word blue is written in, where the color does not match the word that is written. The development of executive control has been seen as early as 30 months (Rothbart, Ellis, Rueda, \& Posner, 2003). Children were presented with two buttons, one to their right and one to their left. Then a picture matching one of the buttons was presented to them either above the right or left button. Children under the age of 30 months had difficulty not attending to the location of the picture instead of the shape and children over 30 months were far more accurate in their responses.

Executive control is important because it has been shown to be associated with greater arithmetic problem solving skills (Passolunghi, Cornoldi, \& De Liberto, 1999). Poor problem solvers were less able to recall relevant information and more likely to recall irrelevant information when solving arithmetic problems. After six experimental phases over two school years Passolunghi and colleagues concluded that the best way to increase problem-solving ability is to somehow reduce the accessibility of irrelevant information.

\section{Measurement}

After reviewing attention from a neuroscience perspective this section will examine how attention has been measured in the fraction literature. The two studies (Hecht et al., 2003; Hecht \& Vagi, 2010) that have assessed attention's effect on fraction performance have done so through the cooperation subscale of the Social Skills Rating System (SSRS; Gresham \& Elliott, 1990). The SSRS is a norm-referenced teacher rating scale that has been shown to correlate with 
direct observations of on-task behavior (Gresham \& Elliott, 1990). If the SSRS is in fact capturing attention it is more of a global estimate of attention than a measure that will give specific information about student deficit. As attention seems to be strongly linked to fraction outcomes and growth in fraction skills Hecht and Vagi (2010) called for a more detailed investigation into the way that attention influences fraction outcomes.

Whatever the SSRS is measuring is strongly related to fraction outcomes, but what if it is not measuring attention at all? Fuchs et al. (2006) state that it is not clear whether teacher behavioral ratings of attention are capturing attention or whether teacher ratings of classroom behavior serve as a proxy for poor academic performance. Fuchs and colleagues conducted another study that showed evidence that teacher ratings of attention were perhaps reflective of poor academic skill in general (Cirino et al., 2007). More research needs to be conducted using attention specific measures instead of the more global on-task SSRS to determine if attention is affecting fraction performance and exactly what aspects of attention account for observed differences.

\section{Summary and Concerns}

So far attention as a factor influencing fraction achievement has only been measured through the SSRS. This study will utilize measures more closely associated with attention to determine the effects of different aspects of attention (e.g., orienting, executive control) on fraction achievement.

\section{Nonverbal Reasoning}

Nonverbal reasoning is the ability to think critically without the use of words or concepts framed in words. Nonverbal reasoning exercises can include pattern recognition, noticing the differences between two pictures or objects finding shapes or objects that don't belong, and 
identifying numerical patterns. Nonverbal reasoning is used in everyday life, judging distance, space, and understanding a person's facial expressions are all examples of nonverbal reasoning.

Nonverbal reasoning has been shown to be a significant predictor of both initial whole number word problem solving ability and word problem solving growth over time (Fuchs et al., 2006; Tolar et al., 2012) but not of whole number computation.

\section{Nonverbal Reasoning as a Predictor of Fraction Performance}

Only Seethaler et al. (2011) has included nonverbal reasoning as a predictor of fraction performance. Interestingly Seethaler et al. found that nonverbal reasoning was a significant predictor of fraction computation skills even though no study has shown nonverbal reasoning to be a significant predictor of whole number calculation skills. Perhaps there is something about the nature of fractions that requires students to have higher nonverbal reasoning skills than needed for similar whole number calculations. It is also important to note that nonverbal reasoning has been linked to whole number word problem solving but that link has yet to be tested for fraction word problems.

\section{Theory}

Little scholarly work has examined nonverbal reasoning as a theoretical construct. Perhaps this is because the idea of nonverbal reasoning is broad and encompasses skills that may or may not have much to do with each other (e.g., reading body language and estimating the number of jelly beans in a jar). Certainly more scholarly work needs to be done examining the different aspects of nonverbal reasoning and their unique contribution to math achievement.

One subcategory of nonverbal reasoning that has been the focus of scholarly work is nonverbal numerical cognition (Gallistel \& Gelman, 2000). Gallistel and Gelman summarized a line of research that clearly shows that human understanding of the magnitude of numbers is 
nonverbal, and that animals share similar understanding of magnitude. For example, in one study participants were briefly shown between seven and 25 items. Participants then had to press a button as fast as they could until they felt that they had reached the number of items presented to them. Participants pressed the buttons an average of eight times per second which is too fast for them to have been silently counting. Researchers received similar results when participants recited 'Mary had a little lamb' wile pressing their buttons. Incredibly researchers conducting similar experiments on animals have found that they produce similar results to human participants. This is important because it means that student understanding of the value of numbers has little to do with the verbal integers we assign to represent each magnitude. Essentially, numbers are nonverbal magnitudes that people try to express through language and labeling. A student with a deficit in nonverbal numerical cognition will have difficulty understanding, interpreting, and making decisions about numbers in math problems.

\section{Measurement}

All studies included in this section (Fuchs et al., 2006; Seethaler et al., 2011; Tolar et al., 2012) assessed nonverbal reasoning through the Wasi Matrix Reasoning test (Wechsler, 1999). The Wasi Matrix Reasoning test measures nonverbal reasoning with four kinds of tasks: pattern completion, classification, analogy, and serial reasoning.

\section{Summary and Concerns}

Nonverbal reasoning has a significant effect on fraction computation (Seethaler et al., 2011), and based on whole number outcomes there is good reason to believe that it will have a significant effect on fraction word problem solving as well (Fuchs et al., 2006; Tolar et al., 2012). In addition to assessing fraction outcomes which have not previously been tested with nonverbal reasoning (i.e., fraction word problem solving, fraction estimation) this study will 
further explore how nonverbal reasoning differentially affects fraction and whole number math skills. Finally, no study has yet examined the differing influence of nonverbal reasoning on students with MD, LD, and their typically developing peers' fraction outcomes.

\section{Extending the Current Line of Research}

Initial research into the conceptual and behavioral factors that influence the development of fraction knowledge in students of varying ability has answered some questions but left several gaps in the research base. The following section will highlight some of the ways in which this study attempts to close those gaps.

\section{Longitudinal Development}

Fraction understanding begins early in schooling and continues to develop at least through the eighth grade (Siegler et al., 2011). Several studies have assessed student fraction knowledge across grade levels in elementary (Hecht \& Vagi, 2012; Seethaler et al., 2011) and middle school (Hecht, 1998; Mazzocco \& Devlin, 2008; Siegler et al., 2011), but no study has crossed the elementary middle school divide using the same measures. Thus there is no way to compare the performance of middle school students to elementary students. One reason this is important is because cognitive processes develop over time so the influence of factors on different deficits may shift through different age groups (Tolar et al., 2012). Another reason that the gap in the current literature is of particular interest because fraction instruction largely ends in the fifth grade. There is some evidence that fraction knowledge continues to develop in middle school (Siegler et al., 2011) but how does the lack of instruction differentially effect students with $\mathrm{LD}, \mathrm{MD}$, and their no identified peers?

\section{Disability versus Difficulty}

Mazzocco and Devlin (2008) demonstrated significant differences between students with 
MD, LD, and their non-identified peers' understanding of fraction magnitudes, yet the current literature base has no studies exploring the differential effects of cognitive factors on the fraction performance of students with disabilities versus those without. In fact only one study in this line of research included students with MD. Hecht and Vagi (2010) found that students with MD performed significantly worse and their fraction knowledge grew at a slower pace than their nonidentified peers. One hundred percent of the difference in fraction performance growth between the MD and non-identified groups was explained by attention and conceptual knowledge of fractions. More research needs to be conducted to better understand the differential effects of cognitive factors on the fraction performance of students who are most at risk. Overall the most vulnerable students' voices are not being heard.

\section{Attention}

Attention has been shown to be a strong predictor of fraction performance, fraction conceptual knowledge, and arithmetic fluency (Hecht et al, 2003; Hecht \& Vagi, 2012), but it has only been assessed through teacher ratings. Though these ratings are a powerful predictor of fraction performance there are still questions as to what aspects of attention the ratings are capturing and if in fact teacher ratings are capturing attention at all (Fuchs et al., 2006). This study aims to provide further data for the role of attention in the development of fraction performance by assessing participants' ability to selectively orient to important information and to filter out irrelevant information.

\section{Nonverbal Reasoning}

When compared to attention relatively little is known about the influence of nonverbal reasoning on the development of math ability. Nonverbal reasoning was shown to be significantly related to fraction computation (Seethaler et al., 2011) even though it had never 
previously been found to be significantly related to whole number computation. Also, nonverbal reasoning has been associated with whole number word problem solving but its effects on fraction problem solving has not yet been assessed. This study intends to extend the current line of research by examining whether nonverbal reasoning independently predicts fraction outcomes and determining what the relative strength of any relationship found.

\section{Research Question}

This study will explore the predictive value of attention and nonverbal reasoning on the fraction performance of students of varying abilities (i.e., LD, MD, non-identified) across grade levels (i.e., forth, sixth, and 8th grades). More specifically this study intended to add to the literature base by exploring the impact of age, disability, and measures of attention and nonverbal reasoning on the fraction performance of students. This study sought to ascertain what extent the course of different groups of students' development of fraction competence is or is not differentially mediated by cognitive factors [the measures of attention and non-verbal reasoning described in the foregoing discussion].

\section{Constructs}

Before describing the methods and assessments used to test this study's hypotheses we will discuss the constructs examined in this study. Clearly defining the constructs examined and how the included assessments align with those constructs is an important part of this research study. Without examining the link between what the research questions and the what is actually being measured interpretation of the results will be limited. For example, as previously mentioned two studies (Hecht et al., 2003; Hecht \& Vagi, 2010) have already examined the impact of attention on fraction performance. Results were significant in both studies but there are questions about whether the measure they used (i.e., SSRS) is actually measuring attention 
(Fuchs et al., 2006; Fuchs et al., 2008). If the SSRS does not measure attention then the interpretation of the results from the study changes completely.

There are four separate constructs that assessed during the course of this study; attention, nonverbal reasoning, the conceptual understanding of fractions, and fraction outcomes. As discussed previously attention has three distinct components that each contribute to "attention" (i.e., alerting, orienting, and executive control). To be able to link attention to student fraction outcomes it is important that the assessment chosen captures one or more of the specific attention components instead of relying on a more general teacher rating of student attention.

Nonverbal reasoning encompasses a large number of skills (e.g., recognizing patterns, reading facial expressions, recognizing differences, understanding inherent values or magnitudes), but this study focused on the area of nonverbal reasoning dealing with students' abilities to recognize patterns, identify differences, and make predictions. These skills theoretically should be linked to students' abilities to understand and manipulate mathematical ideas and have been previously linked to student fraction outcomes (e.g., Seethaler et al., 2011).

The conceptual understanding of fractions encompasses a wide range of skills. Also it is sometimes difficult to separate what types of problems students are solving conceptually or procedurally. Hallet et al., (2010) argued that the same question can be viewed as procedural or conceptual depending on the student's familiarity with the problem type. That being said, for this study the conceptual understanding of fractions is defined as being able to identify and draw representations of fractions, understanding the magnitude of fractions (i.e., placing fractions on a number line, comparing the value of two fractions, identifying equivalent fractions), understanding basic operations with fractions (i.e., adding and subtracting representations of fractions), and understanding how to express fractions in written form (i.e., mixed numbers, 
proper fractions, improper fractions).

Fraction outcomes for this study have been broken into three distinct skills. The first assessment of fraction outcomes will be students' ability to solve basic fraction computation problems (i.e., addition and multiplication of proper fractions, improper fractions, and mixed numbers). The second area of fraction outcomes this study assessed is students' ability to quickly estimate answers to fraction problems without taking the time to actually solve the problem. The third area of fraction outcomes assessed is student ability to read word problems involving fractions and correctly set-up an equation to solve the word problem. 


\section{METHODS}

\section{Research Questions}

This study explored the predictive value of attention, nonverbal reasoning, and conceptual understanding of fractions on the fraction performance of students of varying abilities (i.e., LD, MD, non-identified) across grade levels (i.e., foruth, fifth, and sixth grades). This study aims to answer the following hypotheses.

Ho: Domain-general (i.e., attention, nonverbal reasoning) and domain-specific (i.e., conceptual understanding of fractions) factors uniquely explain significant amounts of variance in fraction outcomes.

Ha: Domain-general and domain-specific factors do not uniquely explain significant amounts of variance in fraction outcomes.

Ho: There are significant differences in fraction outcomes between the fourth, sixth, and eighth grades.

Ha: There are not significant differences in fraction outcomes between grade levels.

Ho: There are significant differences in fraction outcomes between students who have LD, MD, and students who are not identified.

Ha: There are not significant differences in fraction outcomes between students who have LD, MD, and students who are not identified.

Ho: There is a significant interaction between student ability group and grade level (e.g., students 
with LD will be significantly further behind their peers in the sixth grade than in the fourth grade).

Ha: There is not a significant interaction between student ability group and grade level.

Ho: The influence of each domain-general and domain-specific factor will vary as a function of student ability group and grade level.

Ha: The influence of each domain-general and domain-specific factor does not vary significantly as a function of student ability group or grade level.

Ho: The $\mathrm{d} 2$ Test of Attention and results from the cooperation subscale of the SSRS each explain a unique amount of variance in fraction outcomes (i.e., they are not measuring the same thing). Ha: The d2 Test of Attention and results from the cooperation subscale of the SSRS do not explain significantly different amounts of variance in fraction outcomes.

\section{Participants}

This section discusses the process by which schools, teachers, and students were recruited, and how student ability groups were determined. The author of this study sought to create a sample that was representative of students from rural, suburban, and urban backgrounds; that is ethnically diverse; and that represents students from a variety of socioeconomic backgrounds. After receiving IRB approval from the University of Virginia, and from the dissertation committee I began contacting school divisions for potential participation in this study.

Before discussing the participants who did participate in this study I will briefly describe the sampling frame for this study. In 2012 the state of Virginia had 1,856,737 students, of whom 
$56 \%(1,039,275)$ were non-Hispanic White, $21 \%(384,322)$ were African-American, $6 \%$

$(111,068)$ were Asian, $12 \%(221,585)$ were Hispanic, and 5\% $(94,670)$ were of two or more ethnic groups (Kids Count Data Center, 2013). In 2012 the median income for households with children in the state of Virginia was $\$ 73,300$ and across the state $15.6 \%$ of all children were living in poverty. Statewide only $64 \%$ of third grade students passed the math portion of the state-wide standardized assessment (Kids Count Data Center, 2013). This study attempted to recruit a sample that most closely represents the demographic statistics of the state of Virginia.

\section{Selection of School Divisions}

Random sampling of schools for participation in the study would have been ideal, unfortunately in order to use any sort of sampling techniques there would first need to have multiple school divisions willing to participate in the study. The author contacted 14 different school divisions across wide sections of the state of Virginia for potential participation. Many school divisions never replied to initial contac, and others cited concerns about the time of year as the study was taking place in the months surrounding the administration of state standardized testing. Due to the limited number of schools willing to participate this study was unable to utilize any sort of sampling techniques in this study.

One public elementary school $(n=166)$, and one private middle school $(n=130)$ participated in the current study. All students enrolled in participating teachers' classrooms were invited to participate in the study.

The public elementary school that participated had approximately 580 students enrolled from kindergarten through the fifth grade for the 2013/2014 school year, and is located in a rural county in Central Virginia. According to the U.S. Census Bureau (U.S. Census Bureau, 2014) residents in the county in which the study took place have a median income of around $\$ 5,000$ 
less than the state average, but have a slightly lower overall rate of poverty $(8.1 \%$ to $11.1 \%)$. One teacher in each of the fourth and fifth grades was responsible for teaching math to all of the students in her respective grade. Consequently, only two teachers from the school actively participated in the study.

The second school that participated in this study was a large private school enrolling around 1,600 students from kindergarten through the twelfth grade. The private school draws from the population of a city in Central Virginia and four surrounding counties. Unlike a public school, the private school does not draw from the general population and therefore attendees of the school may not represent accurately the general population in which the school lies. Although no individual student financial information was available, approximately $25 \%$ of the students attending the school receive financial assistance. After discussing the purposes and procedures for this study the sixth grade math teacher decided to participate. All participating teachers received a $\$ 50$ gift card for their participation in the study.

\section{Individual Participants}

Partial or complete data were collected for 316 participants but only 296 made it into the final analyses. Most of the participants deleted from the analyses were from the public elementary school and were taking the assessments when a water pipe burst at their school interrupting the testing period and minimizing the number of assessments they could complete. Many of these students simply had too much missing data to reasonably analyze. Consequently 18 participants from the database were deleted due to the burst water pipe. The final two participants were deleted due to their data being influential outliers which will be discussed in a later section of this paper. Demographic information for the participants of this study are provided in Table 1. 
Table 1

Study Demographic Information

\begin{tabular}{|c|c|c|c|c|c|}
\hline & & & Grade & & Total \\
\hline & & 4 & 5 & 6 & \\
\hline \multicolumn{6}{|l|}{ Gender } \\
\hline & Male & $43(51.8 \%)$ & $45(54.2 \%)$ & $66(50.8 \%)$ & $154(52.0 \%)$ \\
\hline & Female & $40(48.2 \%)$ & $38(45.8 \%)$ & $64(49.2 \%)$ & $142(48.0 \%)$ \\
\hline \multicolumn{6}{|l|}{ Ethnicity } \\
\hline & $\begin{array}{l}\text { Caucasian } \\
\text { African- }\end{array}$ & $58(69.9 \%)$ & $59(71.1 \%)$ & $113(86.9 \%)$ & $230(77.7 \%)$ \\
\hline & American & $9(10.8 \%$ & $10(12.0 \%)$ & $4(3.1 \%)$ & $23(7.8 \%)$ \\
\hline & Hispanic & $5(6.0 \%)$ & $9(10.8 \%)$ & $3(2.3 \%)$ & $17(5.7 \%)$ \\
\hline & Asian & $5(6.0 \%)$ & $3(3.6 \%)$ & $4(3.1 \%)$ & $13(4.4 \%)$ \\
\hline & Mixed & $5(6.0 \%)$ & $2(2.4 \%)$ & $6(4.6 \%)$ & $12(4.1 \%)$ \\
\hline & Other & $1(1.2 \%)$ & $0(0.0 \%)$ & $0(0.0 \%)$ & $1(0.3 \%)$ \\
\hline \multicolumn{6}{|l|}{ Disability } \\
\hline & Identified & $11(13.3 \%)$ & $8(9.6 \%)$ & $6(4.6 \%)$ & $25(8.4 \%)$ \\
\hline & At-risk & $4(4.8 \%)$ & $5(6.0 \%)$ & $8(6.2 \%)$ & $17(5.7 \%)$ \\
\hline & Non-Id. & $68(81.9 \%)$ & $70(84.3 \%)$ & $116(89.2 \%)$ & $254(85.8 \%)$ \\
\hline
\end{tabular}

The demographics of this study represent the overall demographics of the areas in which the study was conducted but do not represent the demographics of the entire state of Virginia. When compared to the demographics of schools across the state, this study had an overrepresentation of Caucasian students (77.7\% to $56 \%)$, and an underrepresentation of African-American (5.7\% to $21 \%$ ) and Asian students (4.4\% to $12 \%)$. Further, there was a slight underrepresentation of students with school-identified disabilities included in this study when compared to state averages $(8.4 \%$ to $12.8 \%)$. The school identified disability rate is brought down below state averages due to the inclusion of a private school in the study. Although the private school does work with public schools to offer special education services in their classrooms the overall rate of students with IEPs is below that of public schools.

Determining ability group status. The rate of students receiving special education services across the state of Virginia in 2012 was 12.8\% (VDOE, 2013). Unfortunately all 
students with school-identified disabilities cannot be included in the LD group without any other evidence of math difficulties. This is because these rates represent a variety of different types of disabilities (e.g., learning disabilities, Autism, behavior disorders) and that many students who are identified with LD are done so primarily for reading and not math. Even if different local education agencies reported the number of students they had identified as having a math learning disability the method that each local education agency used to identify students would be different. In light of these difficulties, it is necessary to utilize a standardized math achievement test to identify students as having a math difficulty or learning disability.

All students in participating teachers' classrooms took the math computation portion of the Wide Range Achievement Test 4 (WRAT-4; Wilkinson \& Robertson, 2006). This subtest is a brief standardized assessment that allowed me to divide participants into three different ability levels. Non-identified students were students who scored above the 30th percentile, students with MD were be those who scored below the 30th percentile but had no identified disability, and students with LD were be those who scored below the 30th percentile and had school identified disabilities. It is important to note here that participants in both schools and across all three grade levels scored significantly higher than expected (WRAT-4 M =110). As few students fell in the bottom $30 \%$ based on the provided standardized WRAT-4 scores this study calculated a cutoff of 95 (a little more than one standard deviation below the mean). Out of 296 total participants, 25 were in the LD group, and 17 were in the MD group.

\section{Measures}

This section provides information about all of the measures used in this study. There are four categories of measures: surveys, fraction predictors, fraction outcomes, and an ability grouping test. 


\section{Fraction Predictors}

As developed in previous chapters, multiple factors predict fraction performance, My hypothesis is that attention and nonverbal reasoning work together to effect fraction performance. This section provides descriptions of the measures used to assess attention and nonverbal reasoning.

Attention. Attention has been shown to have a strong relationship with fraction outcomes, fraction conceptual understanding, and arithmetic fluency but it has only been measured through the Social Skills Rating System (SSRS: Gresham \& Elliott, 1990). As discussed previously Fuchs et al., $(2006 ; 2008)$ have questioned whether teacher ratings of student attention capture attention, or whether it is a more general assessment of performance. To help answer questions raised by Fuchs and colleagues, the SSRS and a more direct assessment of attention were included to determine more clearly if there is a significant link between attention and fraction outcomes and if the SSRS is actually capturing student attention.

Social Skills Rating System. Participating teachers answered items from the cooperation subscale of the SSRS (Gresham \& Elliott, 1990) as a global estimate of student attention. Items were filled out based upon student performance during math instruction. Teachers completed the SSRS ratings before students participated in the rest of the assessment. Gresham and Elliott (1990) have found that ratings on the SSRS correlate significantly with direct observation of student behavior and previous research has shown that SSRS scores are significantly related to fraction outcomes (Hecht et al., 2003; Hecht \& Vagi, 2010).

d2 Test of Attention. The d2 Test of Attention is a one-page paper-and-pencil test of attention widely used throughout Europe (Bates \& Lemay, 2004). Each page has 14 rows with 47 "p" and "d" characters in each row (Brickenkamp \& Zillmer, 1998). Each character has one to 
four dashes arranged above and/or below each character. Participants are asked to target the character "d" when it is accompanied by only two dashes. These two dashes can be arranged above, below, or one each above and below the character "d." The character "p" with two dashes above or below serves as a distracter. Participants get 20 seconds to cross out as many of the targeted "d" characters as they can in a single row. Upon completion of one 20 -second trial, participants immediately move to the next row and begin a new trial until all 14 rows have been completed (Bates \& Lemay, 2004).

The $\mathrm{d} 2$ Test of Attention produces several different scores. Scoring of the $\mathrm{d} 2 \mathrm{Test}$ generally reports the total number of characters processed through all 14 trials, errors of omission (i.e., total number of targets not cancelled), errors of commission (i.e., total number of non-target characters cancelled), total errors (i.e., sum of omission and commission errors), percentage of errors (i.e., total errors divided by sum of characters processed), total number of characters processed correctly (i.e., sum of characters processed minus total number of errors), concentration performance (i.e., total errors cancelled correctly minus total number incorrectly cancelled), fluctuation rate in speed (i.e., maximum number of items processed minus minimum number of items processed in a trial), error distribution (i.e., average errors for last four trials minus average errors for first four trials), acceleration (i.e., correlation between trial number and speed), and deterioration (i.e., correlation between trial number and total errors).

It is difficult to isolate the sources of attention deficits (i.e., alerting, orienting, and executive control) in applied settings. Though the $\mathrm{d} 2$ Test does not capture alerting, it captures both orienting and executive control. As discussed previously alerting concerns a person's ability to maintain a state of awareness and focus. Studies assessing alerting measure how much of a benefit students receive (i.e., how quickly they respond) when they are told to look for a stimuli 
in a certain spot. The $\mathrm{d} 2$ Test has no mechanism to gauge how much participants benefit from being told to focus their attention to a particular stimuli. The $\mathrm{d} 2$ Test is assessing how quickly participants can move the spotlight of their attention to new information (orienting), and how quickly they can weed through the distracters (i.e., the letter " $q$ " and any " $\mathrm{d}$ " with 0 , 1 , or 3 dashes; executive control). To be able to separate executive control deficits from orienting deficits it would be necessary to conduct individual computerized assessments of attention (e.g., IVA Computerized Visual and Auditory Performance Test, Test of Variables of Attention). The $\mathrm{d} 2$ Test of Attention was normed on a sample of 6,000 Germans but preliminary psychometric data has since been collected on nearly $600 \mathrm{~K}-12$ and college students in the Unites States (Brickenkamp \& Zillmer, 1998). Across studies conducted in the United States the main score used in this study (CP) has high internal consistency, cronbach's alpha score of .96. Student error rates tend to have lower reliability (Type 1 error $=.80$; Type 2 error $=.79$ ) and are not being used in the main part of this study's analyses. In addition to internal reliability, the $\mathrm{d} 2$ Test of Attention has been shown to have stability over time as test-retest reliability scores generally range from .75 to.95 months after the initial assessment. The authors of the test do point out that more information is needed on the test-retest reliability of atypical populations in the United States.

Brickenkamp and Zillmer (1998) argue for the validity of the d2 Test of Attention in two different ways. First, they argue that at face value the $\mathrm{d} 2$ Test of attention seems to assess the speed and accuracy of selective attention. Selective attention is the ability to quickly key in on important information and ignore or move past irrelevant information. Second, they compared results on the $\mathrm{d} 2$ Test of Attention to several other used tests of attention. In a study of 506 U.S. students the $\mathrm{d} 2$ test of Attention was found to be significantly correlated with the Stroop Color 
Word Test (Golden, 1976), the Trail Making Test A and B from the Halstead-Reitan Neuropsychological Test Battery (Reitan \& Wolfson, 1993), and the symbol digit Modalities Test (Smith, 1982).

Nonverbal reasoning. Student nonverbal reasoning has been linked to fraction calculation (Seethaler et al., 2011), but its influence on other fraction outcomes has yet to be assessed. The researcher created an 18-item, nonverbal reasoning assessment for this study. The assessment was group-administered and participants had no more than 8 minutes to finish. Participants received help clarifying the directions for each section but received no help solving any of the individual problems on the assessment. Nine items on the test present either four or six different figures or groups of figures and asks participants to identify which figure is most different from the other figures. For example, one problem presents five different rectangles of varying size and orientation and one triangle. The triangle is the shape that does not belong as all other shapes have four sides. Five items on the assessment present a series of figures and ask participants to identify what figure would come next. Participants can choose from four different options. For example, one test item presents a hexagon, a pentagon, and a trapezoid. The pattern is that the number of sides on the figure is decreasing by one, so the next figure in the pattern must be a triangle. Three items on the assessment present two figures (e.g., happy face and an arrow) and ask participants what the figures would look like if they were rotated clockwise 90 degrees. The last item on the assessment presents two different circles, each with two lines dividing the circles into quarters. Participants are asked to identify what the circles would look like if they were placed on top of one another.

Conceptual understanding of fractions. As described previously, the conceptual understanding of fractions consists of many different skills. Students' conceptual understandings 
of fractions were assessed through a researcher-created instrument. Included items on the assessment were similar to items used as conceptual tests across a number of fraction studies (Charalambos \& Pitta-Pantazi, 2007; Hecht, 1998; Hecht et al., 2003, Hecht \& Vagi, 2010; Mazzocco \& Devlin, 2008). On the assessment, students will convert pictures to symbols (e.g., 1/4 of a circle shaded, student writes $1 / 4$ ), convert symbols to pictures (e.g., student sees 1/4 and draws a picture representing 1/4), mark the appropriate spot on a number line when given a fraction, compare the size of different fractions (e.g., 3/4, 2/3; which is greater?), add and subtract picture representations of fractions, and identify fractions from sets of objects (e.g., five circles, three are shaded, write a fraction representing the part of the group that is shaded). The assessment consists of 28 problems. Each of the four number line problem was assessed for both the participant's correct placement of the fraction on the number line and the correct division of the number line.

\section{Fraction Outcomes}

This section will discuss the three different types of fraction outcomes being used in this study, fraction calculation, fraction word-problem solving, and fraction estimation. An argument could be made that some aspects of the conceptual understanding of fractions should be viewed as outcomes instead of factors predicting fraction outcomes. For example, the ability to view the representation of a fraction and accurately name the fraction is a fraction skill that we want students to develop. This study included the conceptual understanding of fractions both as an independent and dependent variable instead of simply a dependent variable, because the conceptual understanding of fractions was a large predictor of all other fraction outcomes in previous studies (Hecht et al., 2003, Hecht \& Vagi, 2010).

Fraction calculation. The fraction calculation test consists of 5 addition and 4 
multiplication fraction problems. The multiplication and addition fraction assessments present the 9 problems vertically and are arranged on a single sheet of paper in three columns. The addition assessment includes problems with like denominators and answers that are proper fractions (2), problems with unlike denominators (2), and a problem with mixed numbers (1). The multiplication portion of the calculation assessment were all problems that resulted in proper fractions that needed to be reduced. Students taking the multiplication and addition assessments will be asked to report their answers in simplest form.

Fraction word-problem solving. The fraction word-problem test consisted of 10 word problems that require addition (4), subtraction (2), multiplication (2), division (1), and one that can be either multiplication or addition to solve. Answers were considered correct if students set the problems up correctly and were not required to solve the problems as that would assess fraction calculation.

Fraction estimation. The fraction estimation assessment had 10 items. Students were presented with 10 computation problem (e.g., 9/10 +9/10=) arranged in a column on the left hand side of the test. Ten different answers were placed in a column on the right hand side of the paper. Students then matched the estimated answers with the appropriate question on in the left hand column. These answers were not exact answers to the problems, but were close to the actual solutions. Student responses to each item were recorded and used to generate person ability estimates through item response theory. Pilot testing of researcher created instruments suggested that students would try to solve all of the estimation problems instead of estimating. To combat this I placed a four minute time limit on the assessment and watched the participants to make sure that they are not working out the problems on their paper during this portion of the testing. I came up with the four minute time limit after watching several pilot students who understood 
estimation complete the assessment in fewer than three minutes, while none of the pilot students who did not finish showed any evidence that they understood fraction estimation.

\section{Ability Grouping Measure}

I used the math computation subtest from the WRAT-4 to help determine student ability groups. The WRAT-4 has been found to have reliability from .86 to .90 for a grade-based sample (Wilkinson \& Robertson, 2006). The WRAT-4 has been shown to correlate with several other widely used achievement and cognitive ability measures (e.g., Wechsler Abbreviated Scale of Intelligence, Wechsler Intelligence Scale for Children - Fourth Edition, Kaufman Test of Educational Achievement - Second Edition, Wechsler Adult Intelligence Scale - Third Edition, Woodcock-Johnson Test of Achievement - Third Edition). I will report standardized scores achieved from the WRAT-4 and not grade level equivalence scores. Although accepted and used in research the WRAT-4 is not a powerful measure of student ability due to its short length and limited number of questions. The shortcomings of the WRAT-4 are acceptable for this research study as no high-stakes decisions that impact students are being made with the data.

\section{Procedures for the Study}

In this section I will discuss the procedures I followed to prepare for and carry out this study. I discuss how I prepared materials (i.e., assessments, data collection sheets), and how I gathered demographic and SSRS ratings before conducing the assessment. Then I will discuss the manner in which I actually conducted the assessments in each classroom and finally I will discuss the procedures used for recording the data.

\section{Preparation of Assessment Materials}

Before beginning to collect data researchers created a spreadsheet where to record student demographic information (i.e., grade level, teacher, school, age, gender, ethnicity, special 
education status, socioeconomic status, and ability group), and item-by-item results of all assessment outcomes. Each participant was assigned an identification number and was only identified by that number in the database. Every student participating in the study received a packet containing all of the assessments they needed to complete. The first page of every packet had nothing but a line for the participant's name. The second page had nothing but a line for the participant's identification number. After administration of the assessments and collecting all of the packets the front page of each packet was removed and the assigned identification number was written on the second page. From that point on there was no method of linking student names to any data.

Teachers were given copies of the SSRS to fill out for each of their students. Teachers received these materials when initially meeting with the researcher and the researcher explained how they were supposed to complete all assessments. Teachers were asked to complete the SSRS and demographic information before administration of the other assessments began so that data collection would be finished after administering the other assessments.

I purchased the appropriate test materials for the WRAT-4 and familiarized myself with the testing procedures and scoring before conducting any assessments. According to the publishers of the WRAT-4 the computation subtest can be administered in small groups. All classroom aids and teachers were asked to help monitor small groups of participants while they took the WRAT-4 computation subtest. Participating teachers were asked to recommend participant groupings for administration of the WRAT-4.

The researcher purchased the $\mathrm{d} 2$ Test of Attention and followed all testing procedures as explained in the assessment manual.

\section{Gathering Information}


Before administering other assessments, participating teachers provided demographic information about their students. This data was collected and later matched through participant identification number to the appropriate participant fraction assessment packets.

\section{Conducting the Assessment}

Upon entering each classroom the researcher began by introducing himself and saying the following. "My name is Mr. Bruce and I work at the University of Virginia. Today we are going to do a few activities that will help us figure out all of the things you have learned about fractions. These activities are not a part of your grade but I want you to try your best when you do them because your answers will help us know more about how to be effective teachers."

Administering the WRAT-4. The researcher began the assessment by breaking the class up into three or four groups depending on class size and the number of assistants available to the researcher. Previously established teacher groupings were used in each class. Each assistant was be in charge of monitoring students as they completed the WRAT-4 math computation subtest. The researcher followed the instructions found in the test manual for administering this subtest. The math computation subtest had a time limit of 15 minutes for all students.

Administering the d2 Test of Attention. After completion of the WRAT-4, the researcher administered the $\mathrm{d} 2$ Test of Attention, and followed the procedures set forth in the $\mathrm{d} 2$ manual when administering the test. As described previously the $\mathrm{d} 2$ Test of Attention is made up of 1420 -second individual trials. In each trial participants will have 20 seconds to cross out any "d" they find with two dashes above and/or below it. Each of the 14 rows corresponds to one of the 14 trials and has 47 " $d$ " and "p" characters. After each 20 -second trial, the next trial began immediately meaning that the total administration time should run just below five minutes (four minutes and 40 seconds). 
Administering the timed assessments. Immediately after participants completed the $\mathrm{d} 2$

Test of Attention the researcher had participants complete the two remaining timed assessments. After students complete the d2 Test of Attention students were told the following.

You all did a great job working hard and paying attention during that last activity. Now we are going to do something different. This activity gives you a chance to try and find patterns and solve problems without having to use words. You will have eight minutes to complete as many of the problems in this packet (point to the appropriate pages) as you can. Read the directions for each section before trying to answer each problem. You may ask for help if you do not understand the directions but we will not help you answer any of the problems. Okay, you all have eight minutes to complete as many of these problems correctly as you can. You may begin.

After the eight minutes were up the researcher moved on to administering the brief estimation task. Each participant was told that they would have four minutes to match each fraction addition problem in the left column with the answer that is closest in the right column. The researcher told them that they only had four minutes so they did not have time to actually solve these problems. The researcher informed each class that they could estimate the answers in much less time and then talked out loud through an example of adding 1/16 and 8/9 together.

Administering the untimed assessments. After collection of the timed assessment packets participants worked at their own pace to complete the rest of the assessments (i.e., conceptual fraction understanding, fraction calculation, fraction word-problem solving, and fraction estimation). Directions for each test were written at the top of each individual assessment. Students were told that they were allowed to ask questions relating to the directions of each assessment but that they will not receive help solving any of the individual items. These 
assessments were not timed and each participant was encouraged to answer any question that they were able to. Participants were free to work through this portion of the assessment at their own pace. During this portion of the assessment the researcher walked around the classroom monitoring student progress and collecting participant packets as student complete them.

\section{Recording Data}

At this point the researcher used the teacher demographic sheets to link individual participants to their assigned identification numbers. The researcher did this before leaving the teacher's classrooms so that no student names were taken out of the school building. After linking student names to identification numbers the researcher entered data into the excel spreadsheet using only the assigned identification numbers. After linking data to unique identification numbers the researcher removed the first page of the student assessment packets with participant names on it, and cut the names off of the teacher completed demographic sheets.

Due to my use of item response theory (IRT) to analyze the researcher created assessments, each participants' answers were entered on an item-by-item basis. This resulted in an extensive database stretching hundreds of columns. After entering all of the data $20 \%$ of the participants were randomly selected to have another person score and input their data to assess the reliability of the data scoring and recording process. After the data were all entered into the spreadsheet, all original materials were kept in a locked filing cabinet until the dissertation process is completed at which point the papers will be destroyed.

\section{Analyses of the Data}

In this section the researcher discusses how the data were screened, then the process used to analyze the psychometrics of the assessments utilized in this study, and finally how the data were analyzed to answer my research questions. 


\section{Data Screening}

The data were screened before analyzing either the psychometrics of the assessments used in this study or the results. According to Kline (2011) data need to be checked for extreme collinearity, univariate and multivariate outliers, missing data, and univariate and multivariate normality.

To assess the collinearity of the data the researcher calculated the squared multiple correlation $\left(\mathrm{R}_{\text {smc }}^{2}\right.$. Several multiple regressions with different variables as the criterion and the rest as predictors were used to find $\mathrm{R}_{\text {smc }}^{2} \cdot \mathrm{R}_{\text {smc }}^{2}>.90$ indicates that much of the variance associated with that particular variable is already accounted for by another variable.

Mahalanobis distance (D) was used to assess univariate and multivariate outliers. Mahalanobis distance indicates the distance in standard deviation units between scores for an individual case (vector) and the sample means for all variables (centroid; Kline, 2011). As recommended by Kline conservative level of statistical significance were adopted for this test $(\mathrm{p}<.001)$. Data found to be outliers were checked to see if there was a data entry error or if there was any other error that led to the collecting of bad data. None of the missing data was deemed to be missing due to researcher error.

Kline (2011) suggests using maximum likelihood to deal with missing data. Maximum likelihood is a method of estimating the parameters of a statistical model when measurement of only a sample of the population is known. .

To assess the univariate and multivariate normality of the data this study used the following procedures. The researcher examined the data for univariate normality through visual examination of distributions of the data, and specifically looked for positive or negative skew and whether the data was platykurtic or leptokurtic. Skewness and kurtosis values were 
compared to the skew index and kurtosis index as described by Kline (2011). To assess

multivariate normality Kline suggests the data need to meet three criteria. First, all the individual univariate distributions need to be normal. Second, each variable is normally distributed for each value of every other variable. Third, all bivariate scatterplots are linear and the distribution of the residuals is homoscedastic.

\section{Quality of the Assessments}

I used IRT to analyze the psychometric properties of the researcher created assessments used in this study, and to produce accurate ability scores for each participant. IRT is an alternative to the shortcomings inherent in classical measurement models. First, classical measurement models provide no way to separate student ability from the difficult of the test (Hambleton, Swaminathan, \& Rogers, 1991). For example, two students scoring 50\% on different assessments will most likely not have the same true ability level. IRT on the other hand provides ability scores that are independent of the test being taken and thereby allow comparisons to be made between scores on different tests more easily. Second, reliability in classical measurement theory assumes that parallel forms of the test are equal to each other but there is no easy way to guarantee such equality. Also, the commonly used standard error of the measurement in classical measurement theory assumes that variance is the same for all participants taking a test when in reality variance differs between individual test takers (Hambleton et al., 1991). Third, classical measurement models are test oriented instead of item oriented (Hambleton et al., 1991). IRT provides the ability to analyze at the item level instead of the test level make designing tests targeted for specific purposes easier. 
IRT has two basic assumptions that must be met before being used. First, IRT assumes unidimensionality. A unidimensional assessment measures only one factor, therefore a test that measures both fraction calculation skill and reading ability is not unidimensional. If a test is not unidimensional there is no way to determine the source of variance on any given item (e.g., did the student get the question wrong because he or she could not read or because they did not understand the math?). In reality no test is wholly unidimensional but Hambleton et al. (1991) argue that if one factor is "dominant" in the assessment then the assumption of unidimensionality has been met. For example, a fraction word problem could be considered unidimensional if the reading level required to successfully complete each word problem not an impediment to any of the test takers. Local independence is the second assumption of IRT and is closely related to the concept of unidimensionality. Local Figure 1 independence is the idea that when all Example Item Characteristic Curve influence of the ability or trait being measured is accounted for answers to test items are no longer correlated (Hambleton et al., 1991). If unidimensionality is achieved then local independence will always be found but local independence can be achieved without unidimensionality

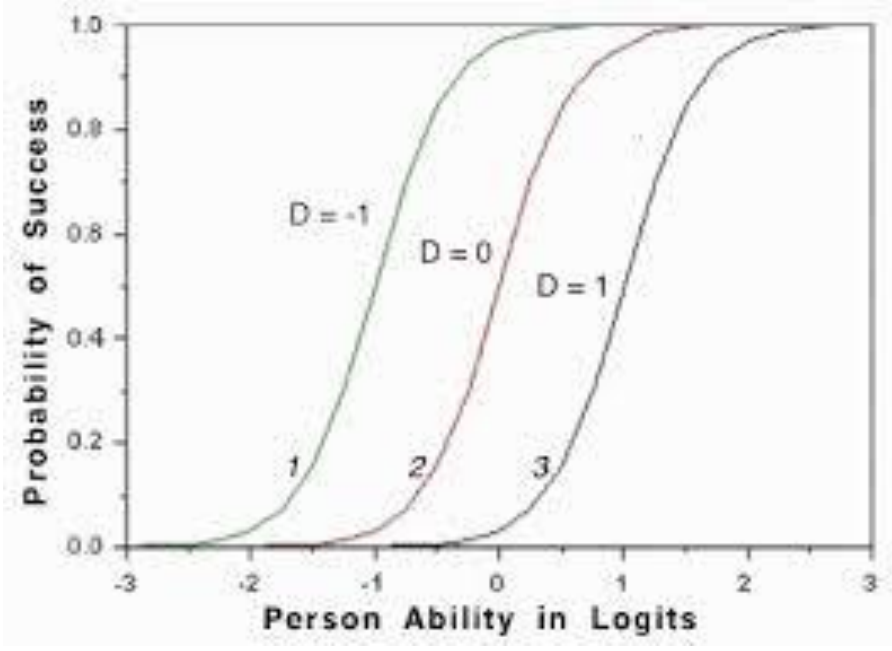
when all latent variables are accounted for.

This study took these two assumptions of IRT into account when creating and analyzing all researcher created assessments. For example, this study sought to assess participants' abilities to read word problems and correctly set-up how to solve each word problem. If students were 
asked "What is $1 / 3$ of $3 / 4$ ?" the skill being assessed is whether the participant could identify this as a multiplication problem; but if student answers to the problem were analyzed then student fraction calculation skill would also be assessed. This would violate unidimensionality so this assessment only asked participants to correctly set-up the word problems without actually having to solve the problems. Another potential threat to unidimensionality for the fraction word problem assessment is the reading level required to solve each problem. Given this potential limitation, word problems were created with simple straightforward language and provided verbal testing for students with severe reading deficits.

IRT creates an item characteristic curve for each question in any given assessment. As seen in Figure 1, the $\mathrm{X}$ axis on each item characteristic curve represents student ability $(\Theta)$ and the $\mathrm{Y}$ axis represents the probability that a student will answer the given problem correctly. Each item characteristic curve is an S shape flattening out at the ends and being the steepest in the middle. Given $\Theta$ for a participant you could predict the probability that he or she would get any individual question correct. The item-by-item analysis of an assessment allows the test maker to analyze the qualities of each item (e.g., reliability, how much information is provided by this question). Another benefit to IRT is that it provides student ability scores independent of the difficulty of the test. This is important as it provides a more refined measure of student ability than simply counting the number of problems a student answered correctly.

There are three main methods of IRT that can be used to assess the psychometric qualities of assessments, the one-parameter logistic model, the two-parameter logistic model, and the three-parameter logistic model. This study utilized a specialized form of the one-parameter logistic model called the Rasch model. The Rasch model was chosen over the other IRT models because the Rasch model assesses whether the data fit the model instead of altering the model to 
fit the data (Andrich, 2004). To put it in everyday terms if one were to weigh a bag of rice that was supposed to weigh 10 pounds on a bathroom scale and the scale said it weighed nine pounds the error could come from one of two places. Either the rice did not weigh 10 pounds or the scale was not measuring accurately. Proponents of the Rasch model argue that altering the IRT model (i.e., two-parameter and three-parameter models) is the same as tinkering with the scale until it says the rice bag is 10 pounds. Proponents of the Rasch model would argue that if the data do not fit then you need to fix your assessment process. Essentially the Rasch model identified whether this study collected good data or if the assessments needed to be altered to obtain better data.

\section{Assessing the Data}

Path analysis. Path analysis was used to analyze the relationships between attention, nonverbal reasoning, and fraction outcomes. Path analysis is an extension of multiple regression that allows for multiple dependent variables and allows for some variables to act as both independent and dependent variables (Norman \& Streiner, 2003). Allowing variables to act as both independent and dependent variables is an important feature of path analysis. On one hand the conceptual understanding of fractions acts as a dependent variable, the result of various cognitive factors (e.g., attention, nonverbal reasoning, and working memory); at the same time the conceptual understanding of fractions influences a variety of fraction outcomes (i.e., fraction calculation, fraction word problem solving, fraction estimation). It is important to note here that path analysis does not use the terms independent and dependent variable due to the fact that one variable can act as both (Norman \& Streiner, 2003). Instead variables are referred to as exogenous and endogenous. Exogenous variables are those that explain variance in other variables but have nothing influencing them included in the model. Inherent traits like age, height, gender, intelligence, and ability level can often be exogenous variables. Endogenous 
variables are any variables that are being influenced by another variable included in the model. All endogenous variables have error (also called disturbance terms) terms attached to them in the path analysis model due to the assumption that all variables are being measured with some degree of error.

In addition to allowing variables to act as both independent and dependent variables, path analysis provides a few other benefits. One benefit is that path analysis is theory driven. Instead of analyzing the data and then trying to fit a theory to the results path analysis begins with theory and tests whether the data fit the theory. The benefit to this approach is that results that are theoretically grounded provide more insight for future practice than data that are not grounded in theory. Another benefit of path analysis is that it will analyze the relationships and interactions between all specified parameters at once instead of having to run numerous separate statistical analyses introducing a large degree of error.

Path analysis is a specific form of structural equation modeling (SEM) without the inclusion of latent variables. Latent variables attempt to explain the influence of factors that have not been directly measured in the study being conducted. For example if a student follows directions, keeps her hands and arms to herself, and interacts in a positive manner wither peers then we could say that she has good "interpersonal skills." We do not actually observe interpersonal skills but we hypothesize that the three behavioral measures we did observe are explained by the concept of "interpersonal skills." One major benefit of SEM over path analysis is that the latent variables account for measurement error and measurement error can greatly impact the reliability of our statistical analysis (Norman \& Streiner, 2003).

Although structural equation modeling would be the ideal form of analysis for this study it was not ideal for the proposed model. The addition of latent variables would dramatically 
increase the number of parameters required in the model (i.e., number of paths + the number of variances of exogenous variables + the number of covariances + the number of disturbance terms) thereby creating the need for a sample size beyond the resources available. If SEM was used for this study it would have suffered from a limited sample size and the statistical power of any analysis would be limited. The use of IRT to generate person ability estimates which were used in the path analysis model is the next best option to SEM and addresses one of the key limitations of traditional path analysis.

In path analysis the researcher proposes a model demonstrating the ways in which the variables relate to each other. The paths between variables are connected by lines with arrows on the end indicating the direction of influence. For example, attention theoretically leads to better student conceptual understanding of fractions, therefore the arrow would point from attention to the conceptual understanding of fractions. All path analysis models must move in one direction, i.e., models must never backtrack (Norman \& Steiner, 2003). Each measured variable also has a disturbance term attached to it to account for measurement error.

In path analysis the number of observations must be greater than the number of parameters (i.e., number of paths + the number of variances of exogenous variables + the number of covariances + the number of disturbance terms). The number of observations can be calculated by the following formula where $\mathrm{k}$ represents the number of variables in the model:

$$
\text { Number of observations }=[\mathrm{k}(\mathrm{k}+1)] / 2
$$

Applying this formula to the model proposed in Figure 2 we find that the number of observations in the proposed model is $(8 \times 9) / 2=36$. Analyzing the proposed model there are 17 paths, two variances of exogenous variables (i.e., for age and ability level), and six disturbance terms of the endogenous variables totaling 25 parameters. Covariance between the two 
exogenous variables which is commonly included in path analysis models was not included because there is no theoretical reason to believe that there is a correlation between age and percentile ranking on a norm-referenced test. As 36 observations are greater than the 25 parameters the proposed model is not over-identified.

Figure 2

Proposed Path Analysis Model

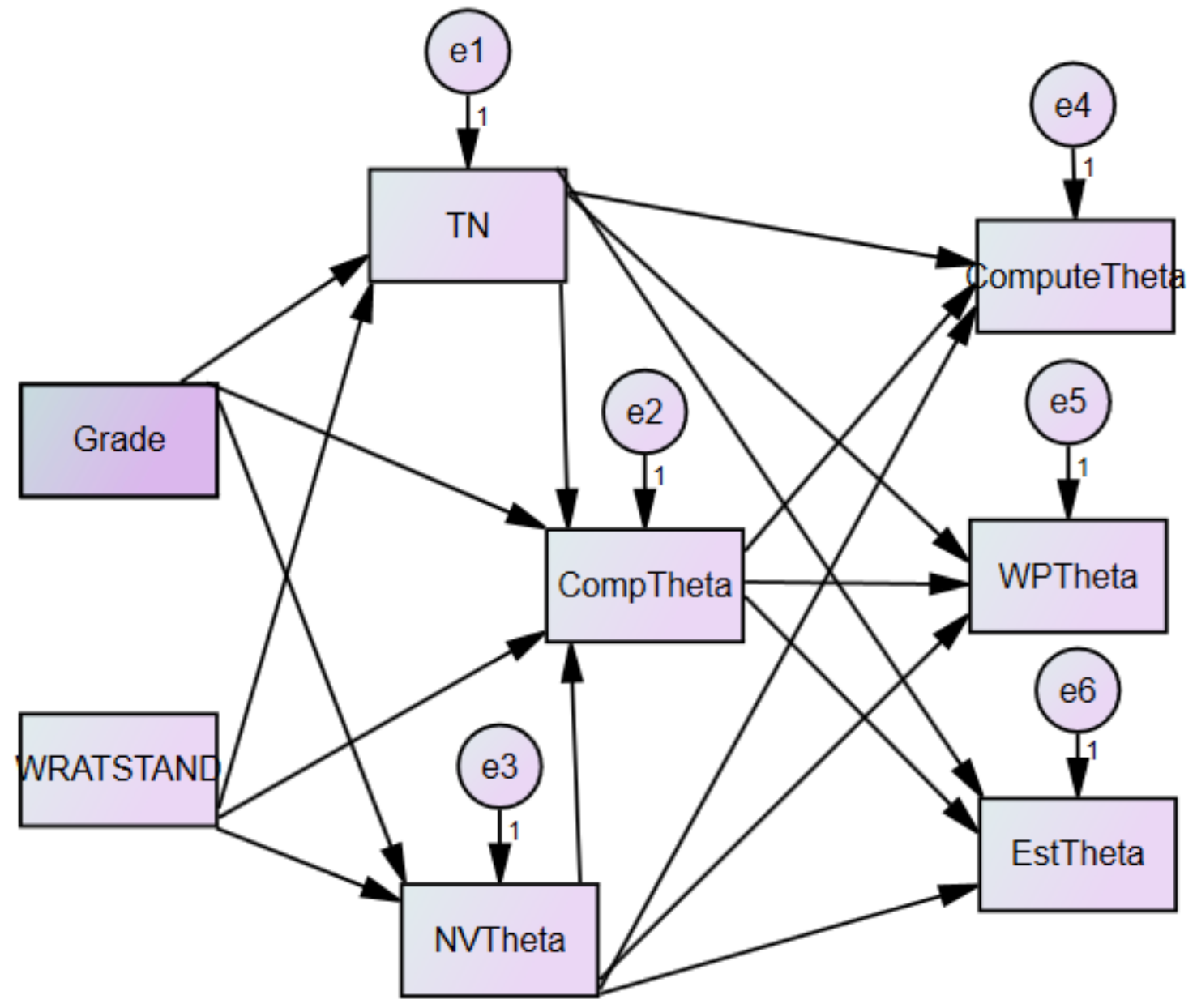

Kline (2011) suggested that the ratio of the number of participants to the number of parameters should ideally be 20:1, and no less than 10:1. As previously calculated the proposed model has 25 parameters and the study should therefore aim to include 500 participants but settle for no fewer than 250 participants. 
Path analysis allowed me to determine whether the data fit my proposed model and to analyze the direct and indirect influence of various factors on participant fraction outcomes. The results section will report both standardized and unstanardized path coefficients for the proposed model. Standardized paths are roughly equivalent to beta weights in a multiple regression model (Norman \& Streiner, 2003). The significance of each path can be checked by dividing the unstandardized direct effect by the standard error creating a z-score for each path (Kline, 2011). In addition to assessing the significance of the direct effect this study will report the signs associated with each path coefficient to determine whether they fit the proposed model.

In addition to reporting the direct effects of the proposed model estimated disturbance variances will be reported. These disturbances reflect the unexplained variability for each endogenous variable (Kline, 2011). By dividing the disturbance variance by the observed variance and subtracting the answer from one this study will be able to report the proportion of variance for each variance explained in the proposed model.

Analysis of the indirect effect of eachvariable included in the proposed model will be reported. Indirect effects can be estimated by multiplying standardized path coefficients with each other. For example, in this study's model if one wanted to know the indirect influence of attention on fraction calculation one could multiply the standardized coefficient for the paths between attention and the conceptual understanding of fractions and between the conceptual understanding of fractions and fraction calculation together (Kline, 2011).

In addition to analyzing the proposed model on a path by path basis this study also ran a goodness-of-fit chi-square test $\left(\chi_{2}\right)$. This tests whether the data are significantly different from the proposed model. A non-significant goodness-of-fit chi-square test indicates that the data fit the model. Chi-square analysis can only say that the data fit the proposed model, the analysis 
cannot say whether the model is correct. If the data fit the model, theory will have to align with the model before adoption. Through these processes each proposed hypothesis will be analyzed. .

Other methods of assessment. Although path analysis will be the backbone of this analysis, other statistical methods will be utilized to answer specific research questions. ANOVAs will be used to examine differences in various fraction outcomes between ability groups (i.e., LD, MD, non-identified), and to examine the interaction effects between ability group and grade level. In addition to ANOVAs partial and semi-partial correlations will be used to examine the relationship between the $\mathrm{d} 2$ Test of Attention and the cooperation subscale of the SSRS which has been used as a measure of classroom attention.

\section{Reliability of Scoring and Entering Data}

A doctoral student score 62 participant packets (21\%) for reliability. Overall, inter-scorer reliability was relatively high for the conceptual understanding of fractions $(97.7 \%)$, nonverbal reasoning $(98.3 \%)$, fraction computation $(98.2 \%)$, fraction estimation $(98.4 \%)$, and the WRAT-4 computation subtest (98.1\%). Reliability was slightly lower for word problem set-up (96.0\%) and the $\mathrm{d} 2$ Test of Attention (93.1\%). Reliability for the d2 Test of Attention was low due to confusion as to whether or not participants had crossed out or erased certain items, and the fact that his study assessed reliability on a line-by-line (14 per assessment) instead of an item-by-item basis (784 per assessment). Disagreements on the $\mathrm{d} 2$ were generally a disagreement of one point on an assessment where the mean was 130.94 and the standard deviation was 26.667 , so disagreements between scorers on the $\mathrm{d} 2$ had very little impact on a participant's overall score.

I next assessed the reliability of entering the data across 97 individual data points for each participant by reentering the data one month after initially creating the database. Agreement between the doubly entered data was high for SSRS scores (100\%), WRAT scores (100\%), the 
d2 Test of Attention (100\%), the conceptual understanding of fractions assessment (99.2\%), the test of nonverbal reasoning (100\%), the fraction word problem set-up assessment (100\%), the fraction calculation assessment (97.7\%), and the fraction estimation assessment (100\%). 


\section{RESULTS}

\section{Data Screening}

The following section describe how the data were screened and prepared for analyses. This study followed the data screening procedures recommended by Kline (2011) in his Principles and Practice of Structural Equation Modeling.

\section{Missing Data}

As with much social science research, this study dealt with missing data across many of the study's assessments. Some of the missing data was due to an unforeseen event during testing (i.e., water pipe bursting at the school during testing), some of it was due to a limited amount of time given to complete the assessments (this was especially a problem at the elementary school where the researcher was given one day to conduct all of the assessments), and some of it may have been due to student inability to answer the questions correctly. All of the included assessments had missing data; WRAT-4 (9.1\%), d2 Test of Attention (4.7\%), nonverbal reasoning $(1.7 \%)$, conceptual understanding of fractions (most items were around $8 \%$ missing), computation of fractions (15\%), estimation of fractions (10\%), and fraction word problem set-up (9.5\%). As mentioned earlier 18 participants were deleted in the fourth and fifth grades who were taking their assessments when the water pipe burst due to their very high rates of missing data.

Remaining missing data were dealt with using the Maximum Likelihood for Incomplete Data (MLE) method at two separate points in my analyses. Maximum Likelihood does not delete participants with missing items or impute the missing data. Instead MLE partitions participants into subsets with similar patterns of missing observations and extracts available statistical 
information (e.g., means, variances) from each subset so all participants are retained in the data set (Kline, 2011). For researcher created assessments this study used MLE in the statistical program jMetrik before creating person ability estimates. This accounted for the missing data in the nonverbal reasoning, conceptual understanding, fraction calculation, fraction estimation, and word problem set-up assessments. For standardized assessments (i.e., WRAT-4, d2 Test of Attention) this study used the MLE procedure in the AMOS structural equation modeling program.

\section{Collinearity}

To assess whether the data was highly collinear a series of multiple regression analyses were conducted, each with a different variable as the criterion and the rest as predictors. The researcher examined the results for any correlations between variables greater than .90 . All correlations fell well below the .90 level indicating that the variables were not redundant. Kline (2011) also recommends checking the variance inflation factor (VIF) to assess collinearity. VIF equals $1 /\left(1-\mathrm{R}^{2}\right)$. If the VIF is score is greater than 10 the one of the included variables may be redundant. None of the VIF scores were greater than 10 indicating that the variables included in this study were not collinear.

\section{Outliers}

Outliers can have an undue amount of influence on the results of statistical analyses. Consequently the data were analyzed for univariate and multivariate outliers. Although there is no single definition of what constitutes a univariate outlier, this study went with the general rule that a univariate outlier lies more than three standard deviations away from the mean (Kline, 2011). For the seven variables included in the path analysis model there were seven univariate outliers. Three participants scored more than three standard deviations below the mean on the 
WRAT-4 calculation subtest. These students were not excluded from the analysis at this time as they were all identified with learning disabilities and represented a population of students who were of particular interest to the purposes of this study. One student scored more than three standard deviations above the mean on the $\mathrm{d} 2$ Test of Attention, and two scored above the cutoff on the nonverbal reasoning assessment. The last outlier was a participant who scored below the cutoff for the comprehension of fractions assessment. All data points were kep as none of the scores were far from the suggested cutoff of three standard deviations and they all represented expected variance in a sample of this size. There were seven identified univariate outliers in a sample of 298 remaining participants, meaning that about $2.3 \%$ of the sample fell outside the third standard deviation which is exactly what you would expect considering the third standard deviation accounts for $97 \%$ of all data. On a more theoretical level, these data points represent real students demonstrating their real abilities and there was not a sufficient reason to delete any of them from further analyses. If participant data had been extremely outside of expected variance then it would have been removed from the dataset. Although no participants were removed from further analysis at this point, univariate outliers were noted to see if they were also multivariate outliers.

Like univariate outliers, multivariate outliers can be significantly influential. A multivariate outlier can result from one or two extreme univariate scores, or when the correlations between variables do not match the pattern of other participants' scores. This study used Mahalanobis distance (D) statistic to assess multivariate outliers. Mahalanobis distance (D) statistic indicates in standard deviations the distance between scores for a participant (vector) and the mean scores for all variables (centroid). Kline (2011) recommends a conservative significance value for this test $(p<.001)$. By this standard, two participants were significant 
multivariate outliers. Both of these cases were flagged as univariate outliers as well.

Closer examination of each participant's scores revealed why these participants did not fit the expected pattern of their peers. The first participant scored extremely high on the $\mathrm{d} 2 \mathrm{Test}$ of Attention (i.e., he/she correctly processed 220 items), but showed an alarmingly high error rate $(30 \%)$. Essentially this student just marked everything as quickly as they could, as such there is no meaningful way to interpret these scores. Consequently this participant was deleted from the dataset. The second participant had the lowest score on the math test of achievement but had above average scores on nonverbal reasoning and fraction calculation and estimation assessments. It is unclear whether this seeming contradiction is due to an error on the part of the researcher (e.g., scoring, inputting data), or if it was something that happened to the participant during testing. As the source of this error is not clear and the participant had already been flagged as a univariate outlier this participant was deleted from further analysis.

\section{Normality}

One of the assumptions of path analysis is that the data display univariate and multivariate normality. This study used a variety of methods to assess univariate normality. Shapiro-Wilk scores were non-significant for the d2 Test of Attention $(p=.399)$, and participants' conceptual understanding of fractions $(p=.009)$ after correcting for family-wise error rate (.05/7). This indicates that participant distributions for these two variables were normally distributed. The other assessments had scores that indicated that the data were not distributed normally $(p<.007)$. As the Shapiro-Wilk test is sensitive to larger sample sizes other methods were also used to assess normality.

Analysis of skewness and kurtosis scores did not indicate any strong violations of normality in the dataset (i.e., all values less than 1 and greater than -1), with the exception of the 
WRAT-4 calculation subtest which had a kurtosis value of 1.024 indicating a slightly higher peak than a normal distribution. Visual inspection of the distributions for each variable revealed that the computation variable and the estimation variable had slight bimodal distributions. Essentially there was a normal distribution for participants who did not understand the task and there were a few students on each assessment who mastered the skill and got all or nearly all of the problems correct. The data were not transformed as the distributions were not typically skewed, and visual inspection generally supported a normal distribution. Further, transformation of the data would make it more difficult to interpret the scores of some of the assessments (i.e., WRAT-4).

Multivariate normality is an assumption of path analysis but there is no standard method to test for it. Kline (2011) offers three criterion that must be met to assume multivariate normality. First, there must be univariate normality. Second, the joint distribution of any pair of the variables is bivariate normal. This means that the distribution of a variable is normal across all values of the other included variables. Third, all bivariate scatterplots are linear, and the distribution of residuals is homoscedastic. Examination of multivariate normality by these criteria did not reveal any obvious violation of multivariate normality.

\section{Linearity and Homoscedasticity}

Normal probability plots for standardized regression residuals were linear and generally had uniform distributions among their residuals. One exception to this was the lower end of the word problem assessment. Participants on the lower end of the ability spectrum tended to score higher than predicted though there were no obvious outliers influencing this drift. Linearity and homoscedasticity for all other variables was supported.

\section{Screening of Assessment Items}


This study used three standardized assessments with known psychometric properties (i.e., d2 Test of Attention; SSRS; WRAT = 4), and four researcher-created assessments. The psychometric properties of the WRAT-4, SSRS, and d2 Test of Attention were previously discussed in the methods section. This section discusses the psychometric properties of the researcher created assessments one at a time.

An item analysis in jMetrik was run for each researcher-created variable. Running an item analysis calculates a discrimination value (i.e., how much information the question provides), general descriptive statistics, and overall assessment reliability. Discrimination scores between 0.3 and 0.7 are ideal but scores between 0.2 and 0.8 are acceptable. Scores outside of those ranges do not provide much valuable information and indicate that the question might not be a good fit for the assessment of which it is a part.

Nonverbal reasoning. The item analysis for the nonverbal reasoning assessment indicated that there was an acceptable, but not great level of reliability for purposes of this study $($ Coefficient Alpha $=.76)$. Examination of individual item discrimination values revealed one item that was not a good fit for the assessment (item 14, discrimination=.14). Examination of the reliability of the test if the item were deleted showed that the reliability of the assessment would increase to .77 . As the increase was negligible the item was retained in the data for further analyses. A DIF analysis was used to determine if any of the items might be biased based on ethnicity or gender. jMetrik provides a letter grade for each individual item, lower grades indicate that the included item might be biased in some way. Examination of DIF scores for gender on the nonverbal reasoning assessment produced $16 \mathrm{~A}$, and two $\mathrm{B}+$ grades. The nonverbal reasoning assessment does not suffer from bias based on gender. Next, items were examined for biased based on ethnicity. Four separate DIF analyses comparing each ethnicity to 
another were run. Percentage of letter grade scores received across all four ethnic comparisons for each assessment are reported here. More detailed information can be found in Table 5 in the appendix.

Across the four DIF analyses for nonverbal reasoning, $88.9 \%$ of all item comparisons received $\mathrm{A}$ grades, $8.3 \%$ received $\mathrm{B}+$ grades, and $2.7 \%$ received $\mathrm{C}+$ grades indicating that there was not evidence of bias in the researcher created test of nonverbal reasoning.

Conceptual understanding of fractions. The item analysis for the conceptual understanding of fractions assessment indicated a high level of reliability (Coefficient Alpha $=$ 9392). All discrimination values were within the recommended range for the assessment and all items were kept for further analyses. All items received A grades when detecting bias for gender. Across the four DIF analyses for nonverbal reasoning, $92.9 \%$ of all item comparisons received A grades, $5.5 \%$ received $\mathrm{B}+$ grades, and $1.6 \%$ received $\mathrm{C}+$ grades indicating that there was not evidence of bias in the researcher created test of the conceptual understanding of fractions.

Computation of fractions. The item analysis for the computation of fractions assessment indicated strong reliability (Coefficient Alpha $=.8920)$. All discrimination values were within the recommended range for the assessment and all items were kept for further analyses. All items received A grades for gender bias. Across the four DIF analyses for nonverbal reasoning, $94.4 \%$ of all item comparisons received A grades, and $5.6 \%$ received $\mathrm{B}+$ grades, indicating that the assessment was relatively free of ethnic bias.

Estimation of fractions. The item analysis for the computation of fractions assessment indicated strong reliability (Coefficient Alpha $=.9123)$. All item discrimination values were within the recommended range and kept for further analyses. All items received A grades for gender bias. Across the four DIF analyses for nonverbal reasoning, $75 \%$ of all item 
comparisons received $\mathrm{A}$ grades, $22.5 \%$ received $\mathrm{B}+$ grades, and $2.5 \%$ received $\mathrm{C}+$ grades. There were significantly fewer items receiving A grades on these comparisons than on the other included assessments, but as the non-A comparisons were almost all $\mathrm{B}+$ grades the variation was not large and further analyses continued.

Word problem set-up. The item analysis for word problem set-up indicated an acceptable level of reliability for the assessment (Coefficient Alpha $=.8585)$. Two items came close to falling outside of the acceptable range for discrimination scores (item 3 , discrimination $=$ .7740 ; item 10 , discrimination $=.2002$ ). This indicates that these two items are not ideal but they are acceptable for the purposes of this study as they are between the .2 and .8 recommended cutoffs. Three items on the word problem set-up assessment received B+ DIF scores for gender while the rest received A grades. Across the four DIF analyses for nonverbal reasoning, $95 \%$ of all item comparisons received $\mathrm{A}$ grades, and $2.5 \%$ received $\mathrm{B}+$ grades, and $2.5 \%$ received $\mathrm{B}$ grades, indicating that the assessment was relatively free of ethnic bias.

\section{Results of the Path Analysis}

This section discusses the findings of this study as they relate to the seven research questions proposed in the methods section. The main analysis will be derived from the results of the proposed path analysis model; as such it is important to discuss how well the model fit the data. A Chi-square analysis of fit was significant, chi-square $(11)=107.43, p<.001$, indicating that the proposed model was not a good fit for the data. A chi-square value of 107 was significantly higher than the critical value of 19.675 at 11 degrees of freedom required to assume the model was a good fit for the data, indicating the model was not even close to being a good fit. As such the researcher had to rethink the proposed model and test alternative models that made theoretical sense. 
After analyzing all the variables in the model and thinking through the theoretical connections between the included variables two changes were made to the initially proposed model. First, originally the WRAT-4 test of achievement predicted growth in attention and nonverbal reasoning. The intention was to use the WRAT-4 scores as a rough proxy for overall intelligence which might explain attention and nonverbal reasoning, but math achievement scores are obviously not the same as intelligence, and it made little sense that math ability would influence attention or nonverbal reasoning. Consequently nonverbal reasoning and attention were changed from endogenous (abilities explained by the model) to exogenous (abilities not explained by the model) variables. Second, grade level was eliminated from the model. Any model that included grade level increased the chi-square statistic by a factor of 10 . Perhaps this is due to the fact that age was measured only by grade level (i.e., 4, 5, 6) and was not normally distributed at all. As the WRAT-4 is standardized for each grade level there was at least some control for grade level in the model if age was removed.

As described in the methods section, in a path analysis model the number of observations must be greater than the number of parameters (i.e., number of paths + the number of variances of exogenous variables + the number of covariances + the number of disturbance terms). The number of observations can be calculated by the following formula where k represents the number of variables in the model:

$$
\text { Number of observations }=[\mathrm{k}(\mathrm{k}+1)] / 2
$$

The newly proposed model shown in Figure 3, has seven observed variables. According to the formula above, the model can handle up to 28 parameters. The model only has 21 parameters indicating that the model is not over identified. Further, Kline (2011) recommends 20 participants per parameter and says at minimum 10 participants per parameter are needed to 
Figure 3

New Path Analysis Model

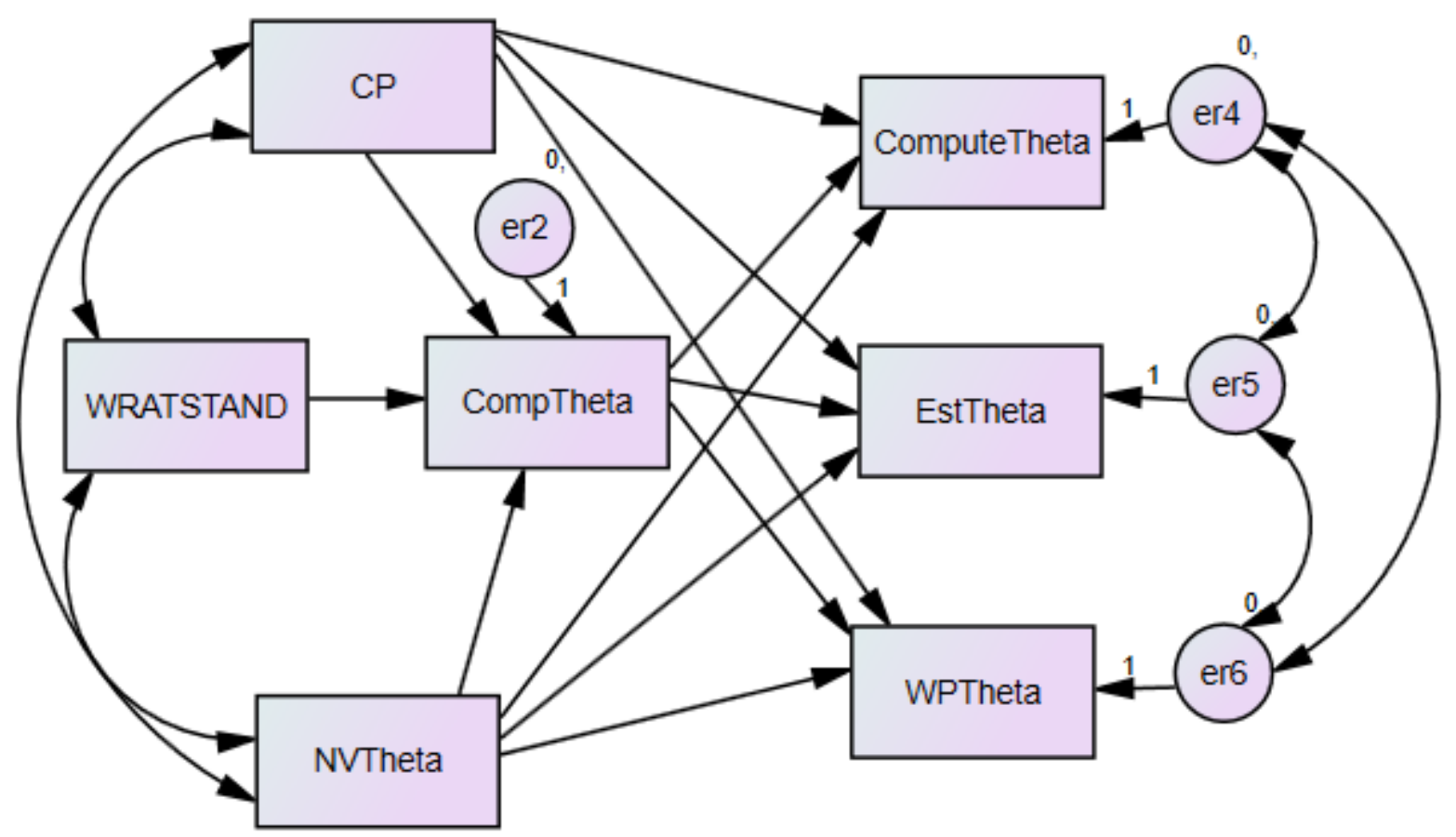

Note: CP stands for Concentration Performance and represents the number of items processed correctly on the d2 Test of Attention

ensure reliable estimates. With 21 parameters, at least 420 participants would be ideal, but the 296 that did participate are well above the minimum of 210 needed for this study.

The new model was a relatively good fit for the data, chi-square $(3)=9.033, p=.029$. As the Chi-square statistic indicated a decent fit, other measures of fit were examined as well. The Normed Fit Index and Comparative Fit Index indicate the proportion of improvement of the overall fit of the model relative to a model where independence is assumed. The closer values are to one, the better the model explains the data. The Normed Fit Index (.987) and Comparative Fit Index (.991) values indicate that the model is a strong fit for the data. Last,the Root Mean Square Error of Approximation (RMSEA) was examined, which evaluates the extent to which a model fails to fit the data per degree of freedom. Any value over 0.1 is considered a bad fit. The proposed model had a RMSEA score of .08 indicating that the model fit the data. Taken in total, 
the analyses indicated that the model was a good fit for the data; therefore, the analyses proceeded.

Ho: Domain-general (i.e., attention, nonverbal reasoning) and domain-specific (i.e., conceptual understanding of fractions) factors uniquely explain significant amounts of variance in fraction outcomes.

The use of path analysis in this study allows the examination of the amount of variance explained by each predictor variable while controlling for the influence of all other variables.

\section{Attention}

As seen in Table 2, attention explained a significant amount of unique variance in fraction conceptual understanding, and had a moderate standardized regression weight of .256. Attention was also a significant predictor of fraction computation, fraction estimation, and word problem set-up. Standardized regression weights for calculation, estimation, and word problem set-up were not large, indicating that the relationship is significant, but that attention is only a small part of what predicts these fraction outcomes. Examination of the standardized indirect effects of attention mediated through the conceptual understanding of fractions to fraction calculation (.134), fraction estimation (.103), and word problem set-up (.110) indicate that the total effects of attention on fraction outcomes is moderate after accounting for the influence mediated through the conceptual understanding of fractions.

\section{Nonverbal Reasoning}

Nonverbal reasoning scores explained a significant amount of variance in the conceptual understanding of fractions, fraction estimation, and fraction word problem set-up, but were not significantly related to fraction computation. This result contrasts with the results of Seethaler et al. (2011) who found that nonverbal reasoning was linked to fraction calculation. Estimation of 
standardized regression weights for the influence of nonverbal reasoning on fraction comprehension, fraction estimation, and word problem set-up were moderate, while the standardized regression weight for fraction computation was low. Examination of indirect effects standardized regression weights revealed that the influence of nonverbal reasoning on fraction computation (.139), estimation (.236), and word problem set-up (.224) was partially mediated through the conceptual understanding of fractions. Combination of the indirect and direct effects of nonverbal reasoning on fraction outcomes reveals a moderate strength relationship between nonverbal reasoning and fraction estimation and word problem solving.

Table 2

Structural coefficients in path analysis model (standard errors in parenthesis)

\begin{tabular}{llll}
\hline Path & Unstandardized & Standardized & p value \\
\hline & & & \\
Links to conceptual understanding & & & \\
1. WRAT-4 - conceptual & $.061(.007)$ & 0.389 & $\mathrm{p}<.001$ \\
2. Attention - conceptual & $.019(.003)$ & 0.256 & $\mathrm{p}<.001$ \\
3. Nonverbal - conceptual & $.469(.083)$ & 0.266 & $\mathrm{p}<.001$
\end{tabular}

Links to fraction calculation
4. Attention - calculation
$.013(.004)$
0.154
$\mathrm{p}=.002$
5. Conceptual - calculation
$.597(.062)$
0.524
$\mathrm{p}<.001$
6. Nonverbal - calculation
$.113(.103)$
0.056
$\mathrm{p}=.273$

Links to fraction estimation

$\begin{array}{llll}\text { 7. Attention - estimation } & .010(.005) & 0.108 & \mathrm{p}=.045 \\ \text { 8. Conceptual - estimation } & .503(.074) & 0.403 & \mathrm{p}<.001 \\ \text { 9. Nonverbal - estimation } & .351(.122) & 0.159 & \mathrm{p}=.004\end{array}$

Links to word problem set-up

10. Attention - word problem

$.010(.004)$

0.12

$\mathrm{p}=.017$

11. Conceptual - word problem

$.478(.061)$

0.431

$\mathrm{p}<.001$

12. Nonverbal - word problem

$.423(.101)$

$\mathrm{p}<.001$

\section{Conceptual Understanding of Fractions}

Math achievement, attention, and nonverbal reasoning explained $45.1 \%$ of the observed 
variance in the conceptual understanding of fractions. In turn the conceptual understanding of fractions explained a significant amount of variance in fraction calculation, estimation, and word problem set-up. As seen in Table 2, the standardized regression weights from the conceptual understanding of fractions to all three fraction outcomes are all larger than .4 indicating a fairly strong relationship between the variables.

\section{Ho: There are significant differences in fraction outcomes between the fourth, fifth, and sixth grades.}

After eliminating grade level from the model, the researcher had to find alternative methods of assessing the influence of grade level on fraction performance. This was achieved by examining the frequency and types of fraction errors that students made in the fourth, fifth, and sixth grades. Although there were many different types of fraction errors made by participants in this study there were eight mistakes that occurred with more frequency than others. Figure 4 provides visual examples of some of the more common mistakes observed in this study.

Unit of measurement. In order work with fraction problems, participants must first understand the unit they are working with (Lamon, 2012). For example, telling someone they should put three portions of sugar in their cookie recipe has no meaning until you understand what size cup you are using to measure the three portions of sugar. Understanding the unit of a given fraction is similar to understanding what size measuring cup needs to be used. A common mistake participants made was not representing the unit of measurement when working with factions. Students making this mistake had difficulty working with fractions greater than one. For example, in one question participants were asked to draw a representation of 5/4. Participants getting this item wrong either divided one whole into five parts or drew one whole and had one little piece separate from the whole. The trouble with the second representation is that one piece 
Figure 4

Examples of Common Student Fraction Errors

Unit of Measurement
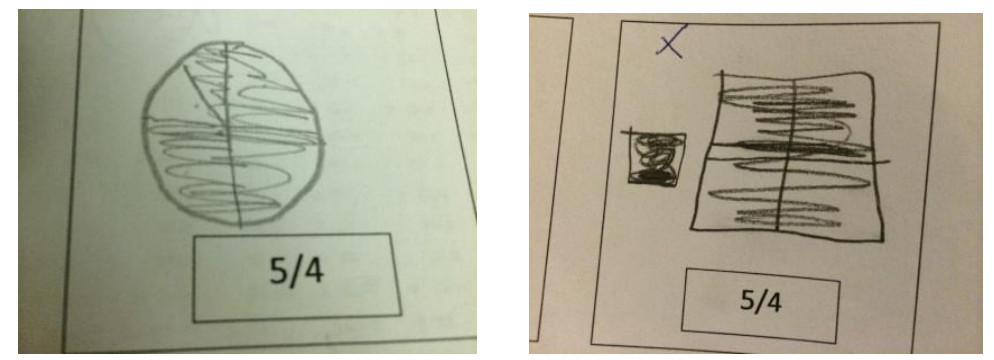

Equal Partitioning
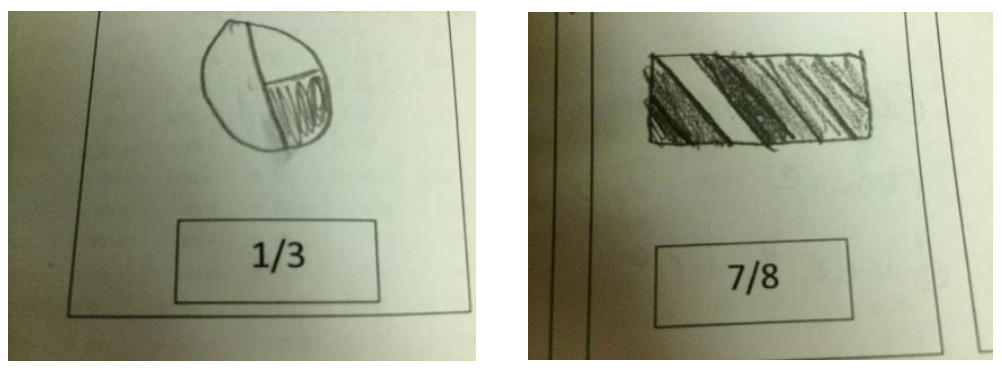

Value on the Number Line
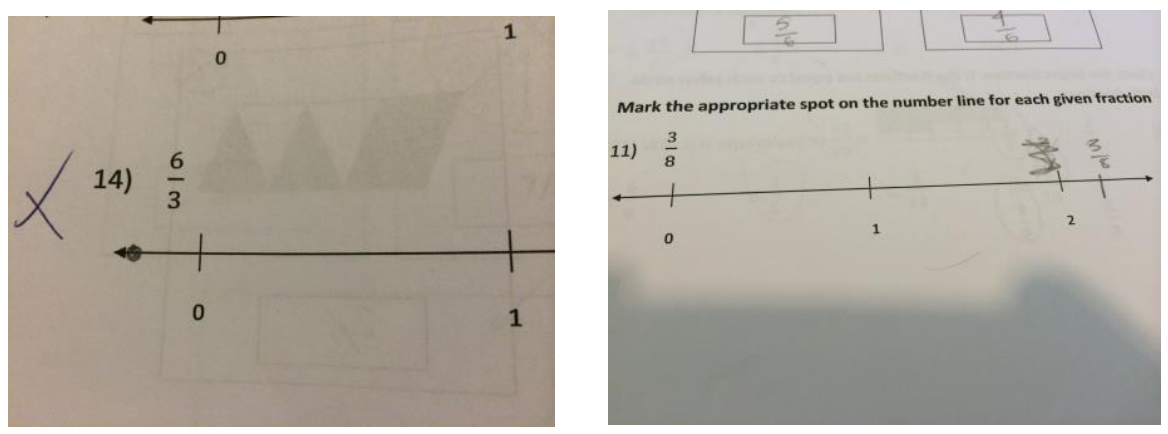

Partitioning of the Number Line

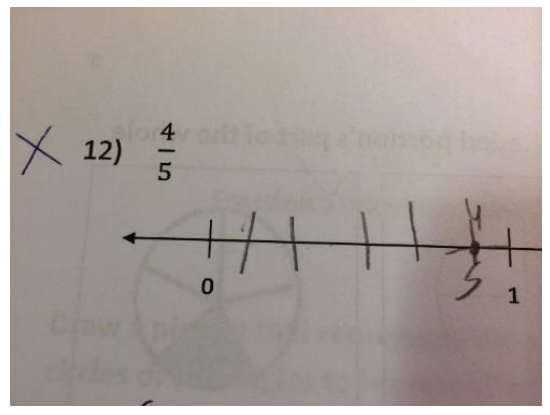

\section{Mark the appropriate spot on the $n$}

11) $\frac{3}{8}$

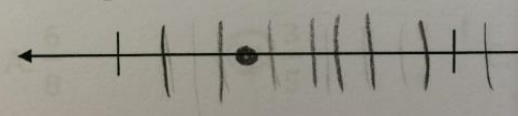

0 
on its own does not represent the $1 / 4$ of the second whole, the other three pieces are necessary as it takes four pieces to represent the second whole. As can be seen in Figure 5, the percentage of participants making this mistake gradually decreased from the fourth through the sixth grades, $\operatorname{chi}$-square $(2)=21.52, p<.001$.

Equal partitioning of fractions. Though not as common as other errors a subset of participants had difficulty with the concept that fractions need to be divided into equal, nonoverlapping pieces. Around 15\% of participants across all grade levels made this type of error. This was the only error that sixth graders did not perform better than their younger counterparts without any explanation (see note of the addition of fractions).

Simplifying. There is debate among mathematics educators as to whether or not it is important to have students simplify fractions in the early stages of fraction learning (Lamon, 2012). Simplifying a fraction after solving some other type of problem is adding an unnecessary extra step that could possibly confuse fraction learners. Certainly there are times in more advanced mathematics courses where rational numbers should not be simplified. In spite of these objections to requiring simplification, students in participating classrooms were asked to simplify all fraction items they worked with, and participants were given oral directions to simplify during this assessment. Although there is debate over the use of simplification, simplifying a fraction can help a student more easily grasp the value of a rational number. For example, 12/32 is difficult more difficult to conceptualize than $3 / 8$. Three and eight are numbers students are more familiar with and would have an easier time proportionally comparing their values.

Though not exactly an error, forgetting to simplify was one most common reasons participants did not arrive at the desired answer. Participants in the sixth grade simplified more often than students in the fifth and fourth grades, chi-square $(2)=17.293, p<.001$. Even so, as 
seen in Figure 5, over half of the sixth grade participants forgot to simplify multiple answers throughout the assessments.

Value on the number line. The first of two number-line-related errors was the value of a fraction on a number line. Examination of Figure 5 indicates that students' abilities to correctly locate values on a number line improves over time, chi-square $(2)=45.495, p<.001$, but a longitudinal analysis would need to occur in order to support those claims fully.

Partitioning of the number line. The most common error made by participants across all grade levels was the improper partitioning of number lines. Participants were asked to represent several fraction values on a number line (e.g., 4/5, 6/3) and were asked to partition the number line to show why they placed the fraction where they did on the number line. Eighty-five percent of fourth grade students were unable to complete this task. Although there was improvement in the fifth (78\%) and sixth grades (71\%) significant numbers of students continued to struggle with this skill. A large portion of the participants displayed little knowledge of how to correctly divide a number line, but several students came close. Students who demonstrated that they understood the general concept but had not mastered it tended to divide the number line into one too many spaces. For example, when representing $4 / 5$ they drew five lines, making six spaces on the number line. Participants making this mistake do not understand that the unit of measurement corresponds to the number of segments on a number line, not the number of lines partitioning the number line. Another eight students made the error of correctly dividing the number line and then placing the fraction between two points on the number line. Participants making these two types of errors appear to have an emerging mastery of this skill.

Comparing the value of fractions. Students making this error failed to compare the 
relative value of four pairs of fractions consistently. Participants in the sixth grade performed much better on this task than students in the fifth and fourth grades, $\operatorname{chi}$-square $(2)=27.636, \mathrm{p}<$ .001 .

Equivalent fractions. Participants making this error were unable to identify equivalent fractions correctly when provided with four options. Like with other errors, participants in the fourth grade were far more likely to make this error than their older counterparts.

Figure 5

Percent of Fraction Difficulties across Grade Level and Skill

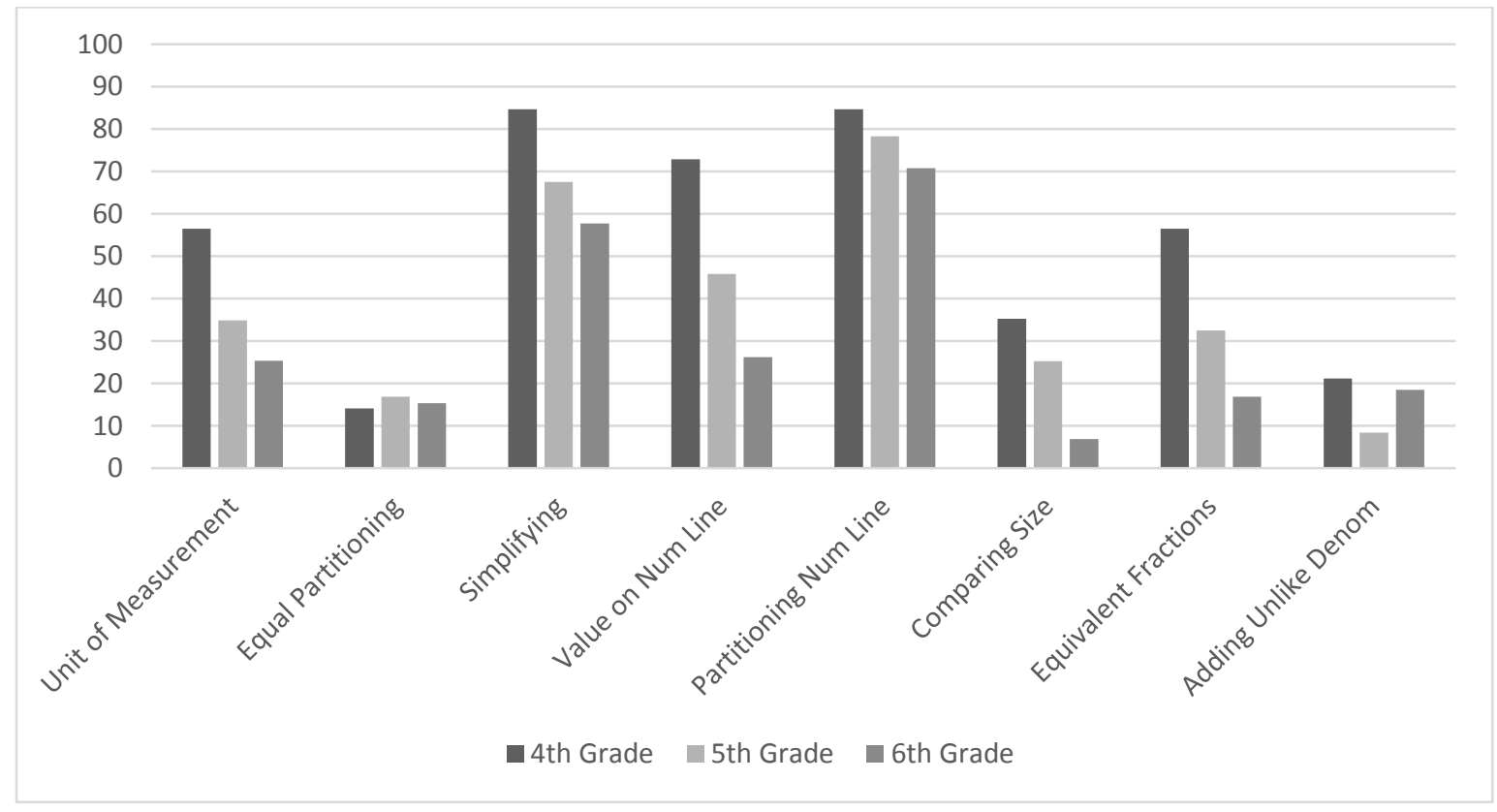

Adding unlike denominators. This error, like dividing fractions into equal portions, did not improve much as participants got older. An understanding of what was going on in the sixth grade classroom at the time of the assessment can help to explain their higher error rate for the addition of fractions than their fifth grade peers. At the time the fraction assessments were administered, the sixth grade students had just finished a long unit on multiplying fractions and many of the student applied multiplication procedures to addition problems. Although they still answered the addition problems incorrectly the recent teaching of multiplication procedures 
helps to explain the increased error rate.

\section{Ho: There are significant differences in fraction outcomes between students who have LD, MD, and students who are not identified.}

The impact of student ability on fraction outcomes was examined in two different ways. First, the relationship between WRAT-4 Calculation scores and the conceptual understanding of fractions was examined as the WRAT-4 scores were used to place students in ability groups. The WRAT-4 Calculation subtest scores were a significant predictor of student conceptual understanding of fractions, $\mathrm{b}=.061, \mathrm{p}<.001$. The standardized regression weight of the relationship between math achievement and the conceptual understanding of fractions was .389 which indicates a fairly strong relationship between math ability and the conceptual understanding of fractions. As WRAT-4 scores were used to separate participants into nonidentified, MD, and LD groups the fact that math achievement scores are significantly linked to fraction comprehension indicates that there will probably be differences in fraction outcomes among the ability groups.

As discussed in the methods section, participants were divided into three ability groups: those with school identified disabilities who also scored in the bottom $30 \%$ on the WRAT $-4(\mathrm{n}=$ 25), those who scored in the bottom $30 \%$ on the WRAT- 4 and were not identified with a disability $(n=17)$, and those who scored above the $30^{\text {th }}$ percentile. Statistical differences explained by ability grouping on the WRAT-4 were not analyzed as WRAT-4 scores were used to help form the groups themselves.

A series of ANOVAs controlling for the grade level of each participant were ran to determine if there were significant differences in variable outcomes explained by ability grouping. There was a significant main effect for $\mathrm{d} 2$ Test of Attention scores explained by ability 
grouping, $F(2,278)=7.144, p=.001, \eta^{2}=.047$. Almost $5 \%$ of the observed variance in attention was accounted for by ability group status after accounting for grade level. Post hoc group contrasts revealed that non-identified participants $(M=133.2)$ correctly processed significantly more items than students in the MD $(M=90.41, p=.002)$, and LD groups $(M=89.4, p=.024)$. There were no significant differences between the low-achieving and LD groups $(p=.322)$. Visual analysis of differences in persona ability estimates based on ability group status are provided in Figure 6. Overall the differences in attention scores between groups are visible but not large.

There was a significant main effect for nonverbal reasoning person ability estimates explained by ability grouping, $F(2,292)=10.117, p<.001, \eta^{2}=.070$. Seven percent of the observed variance in nonverbal reasoning scores was accounted for by ability group status. Post hoc group contrasts revealed that there was no significant difference between the non-identified group $(M=1.14)$ and the MD groups $(M=.84, \mathrm{p}=.220)$. Participants in the LD group $(M=.05)$ scored significantly lower than participants in the MD group $(\mathrm{p}=.049)$, and the non-identified group $(p<.001)$. The fact that participants in the LD group scored significantly lower than their MD peers indicates that nonverbal reasoning is potentially a deficit that explains observed differences in fraction outcomes.

There was a significant main effect for the conceptual understanding of fractions person ability estimates explained by ability grouping, $F(2,292)=35.243, p<.001, \eta^{2}=.191$. Nineteen percent of the observed variance in the conceptual understanding of fractions was explained by ability group status after controlling for grade level. Post hoc group contrasts revealed significant differences between the non-identified group $(M=1.21)$ and both the $\mathrm{MD}(M=-.03, p=.001)$ and LD groups $(M=-1.75, p<.001)$. There were also significant differences between the MD 
and LD groups $(p=.010)$. These results indicate that students with identified learning disabilities have significant conceptual fraction deficits not explained by general math achievement.

There was a significant main effect for computational fraction person ability estimates explained by ability grouping, $F(2,292)=10.114, p<.01, \eta^{2}=.065$. Six and a half percent of the observed variance in the computation ability estimates of participants after controlling for grade level was explained by ability group status. Post hoc group contrasts revealed that participants in the non-identified group $(M=0.86)$ scored significantly higher than their MD $(M$ $=-.47, p=.002)$, or LD counterparts $(M=-1.03, p=.001)$. There were no significant differences between participants in the low-achieving or LD groups $(p=.890)$.

There was a significant main effect for estimation fraction person ability estimates explained by ability grouping, $F(2,292)=6.434, p=.002, \eta^{2}=.042$. Just over four percent of the observed variance in the estimation ability after controlling for grade level was explained by ability group status. Post hoc group contrasts revealed no significant differences between participants in the $\operatorname{LD}(M=-1.36)$ and MD groups $(M=-.424, p=.589)$. There were however significant differences between participants in the non-identified $(M=.56)$ group and those in the $\operatorname{MD}(p=.050)$ and $\operatorname{LD}(p=.002)$ groups.

There was a significant main effect for word problem set-up person ability estimates explained by ability grouping, $F(2,292)=33.475, p<.001, \eta^{2}=.187$. Almost $19 \%$ of the observed variance in word problem set-up after controlling for grade level is explained by ability group status. Post hoc group contrasts revealed that participants in the LD group $(M=-.82)$ scored significantly lower than their peers in the MD $(M=1.02, \mathrm{p}=.014)$ and non-identified $(M$ $=2.44, p<.001)$ groups. The difference between the MD and non-identified groups was also significant $(p=.001)$. 
Figure 6

Fraction Skill Person Ability Estimates by Ability Grouping

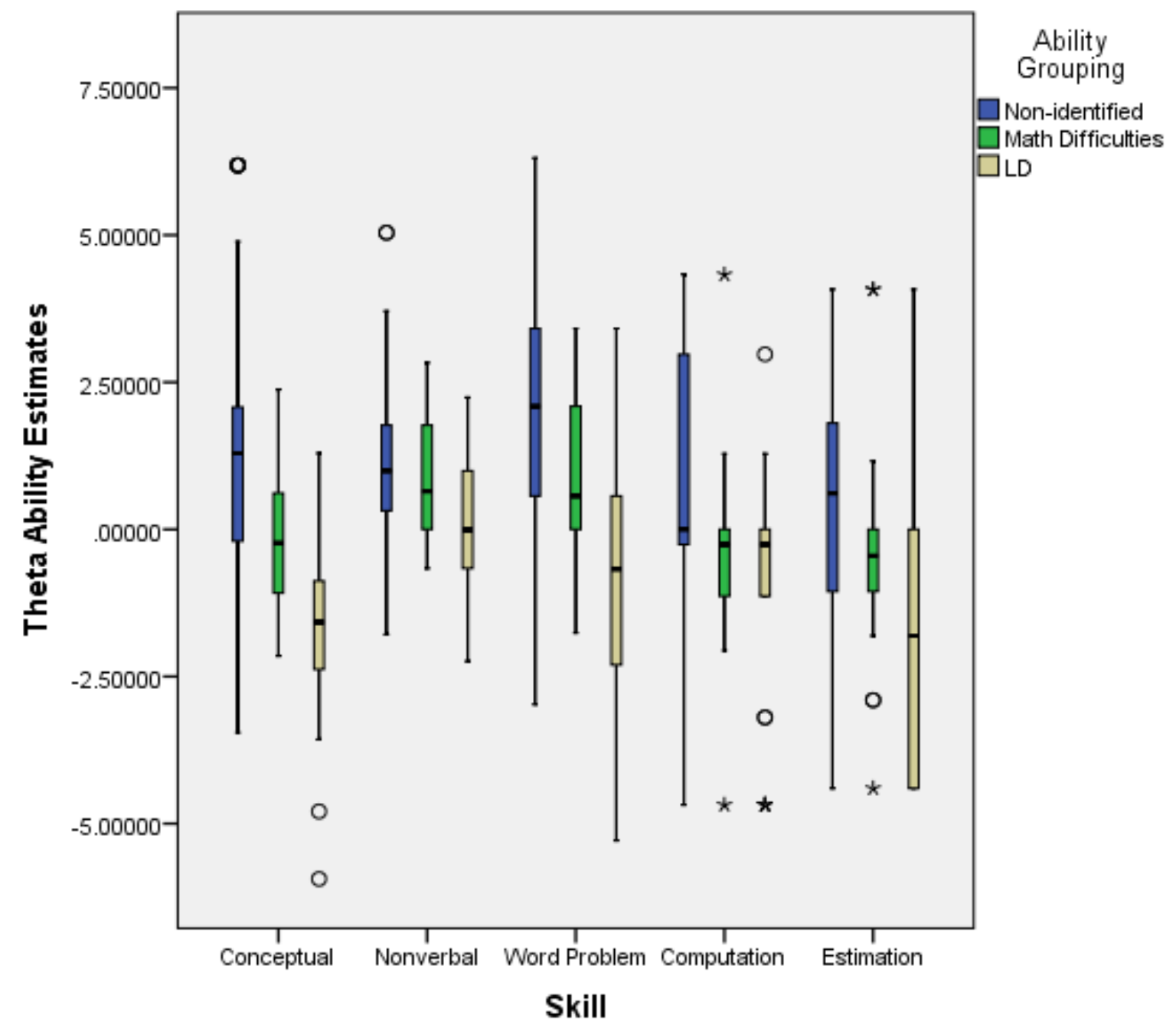

Ho: There is a significant interaction between student ability group and grade level (e.g., students with LD will be significantly further behind their peers in the sixth grade than in the fourth).

Although grade level has an important effect on fraction development the evidence collected in this study does not support a strong interaction effect between disability and grade level. Examination of the graphs in Figure 7 show that the differences between identified and non-identified participants in the fourth grade are relatively the same in the sixth grade. The one 
exception to this is that the fraction calculation ability estimates of non-identified students in the sixth grade appear to be much higher than their MD and LD peers. This difference may be due to non-identified participants in the sixth grade performing much better on the few multiplication problems included in the assessment than their peers in the MD and LD groups. A better method to answer this question would be through a longitudinal study tracking the fraction knowledge growth of participants across years of fraction learning.

Figure 7

Grade Level by Ability Group Interaction

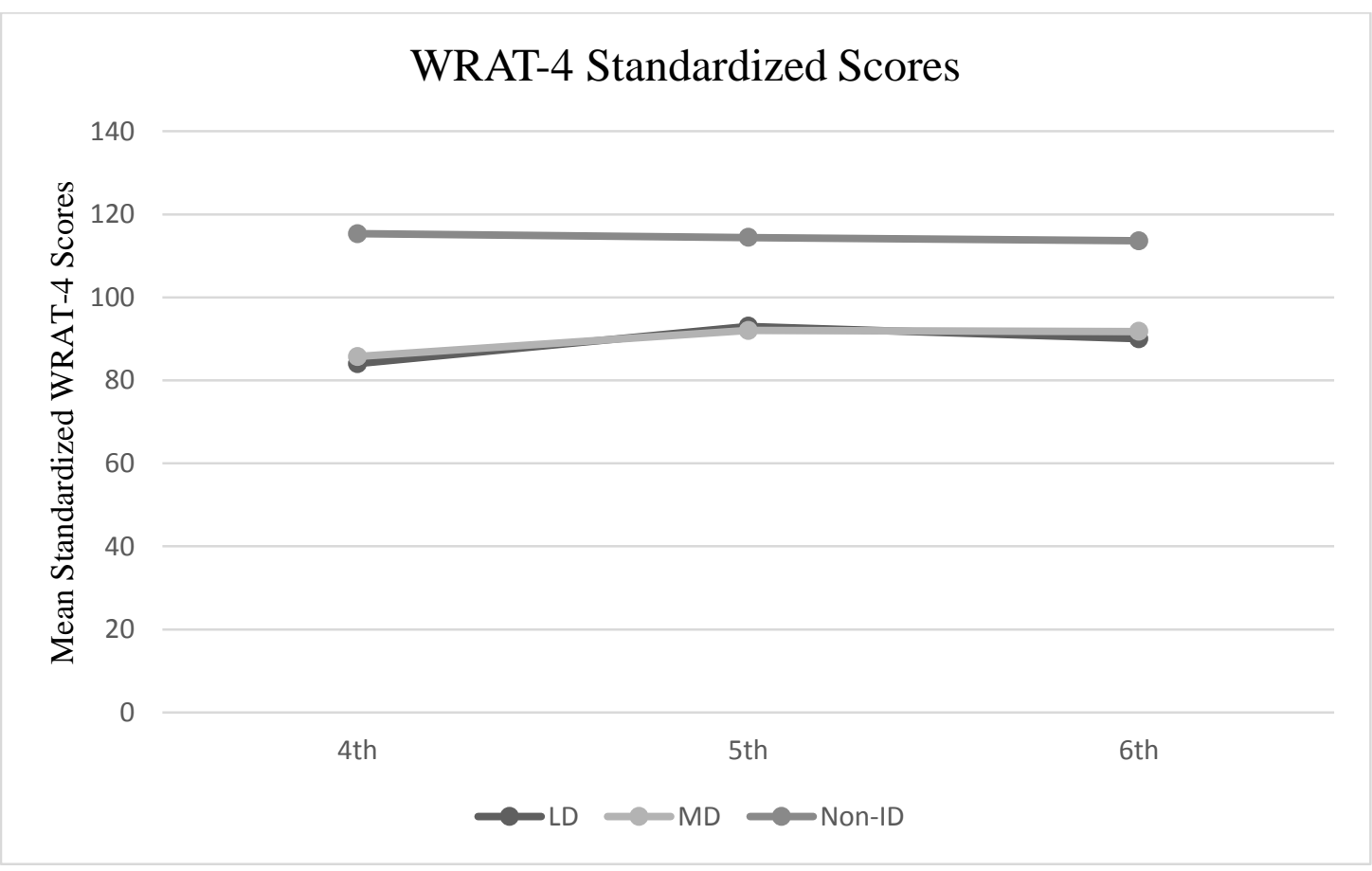


Figure 7 cont.
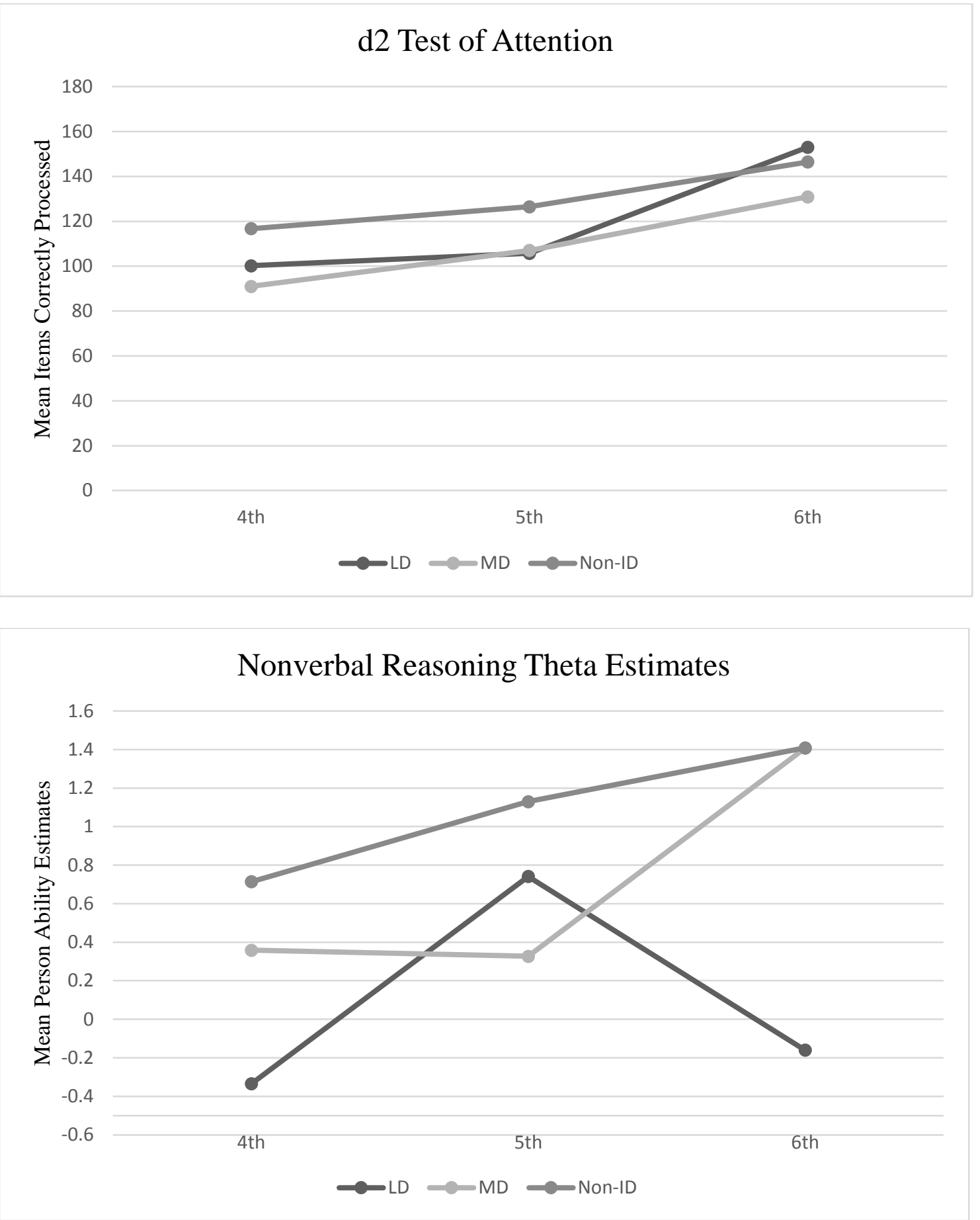
Figure 7 cont.
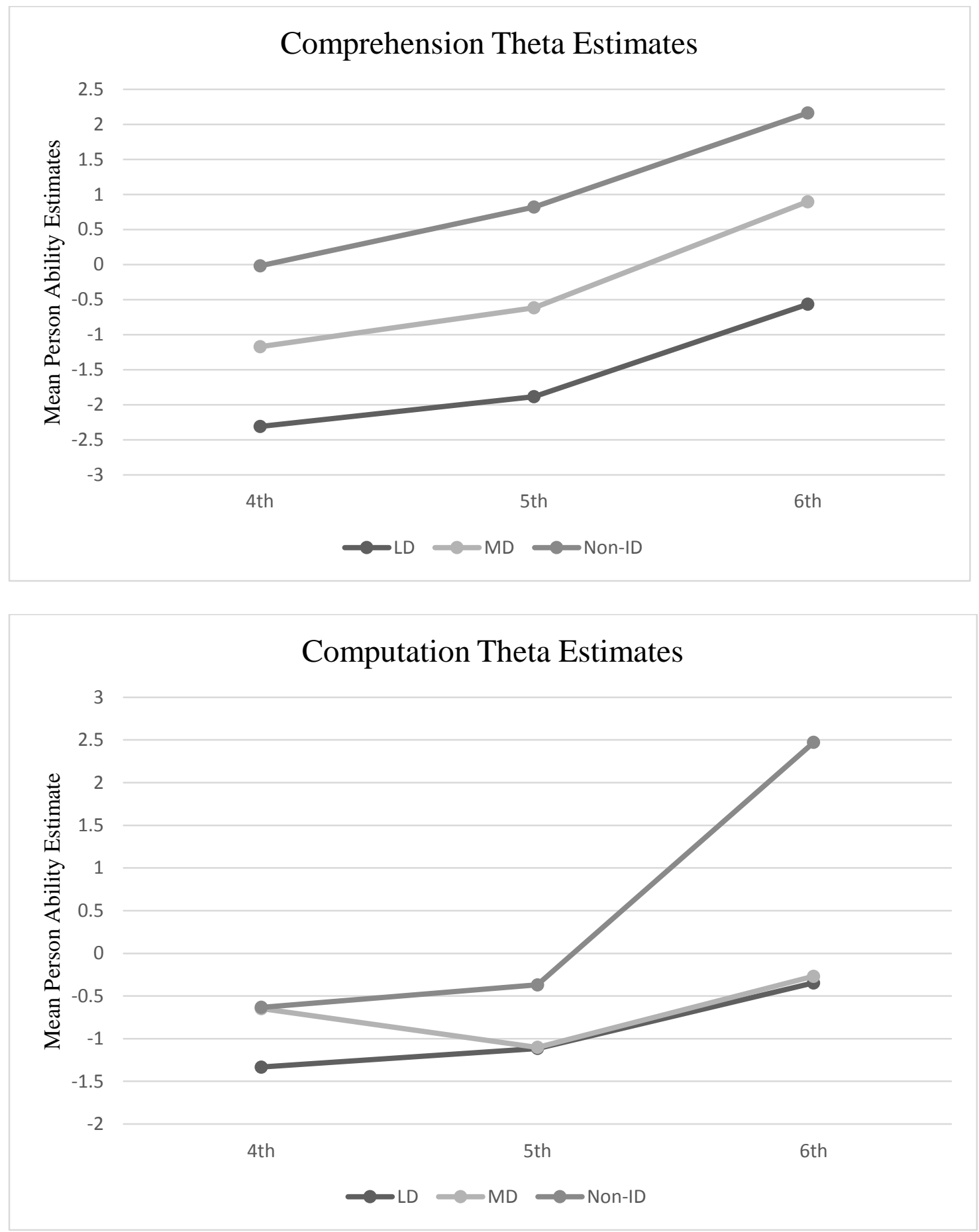
Figure 7 cont.
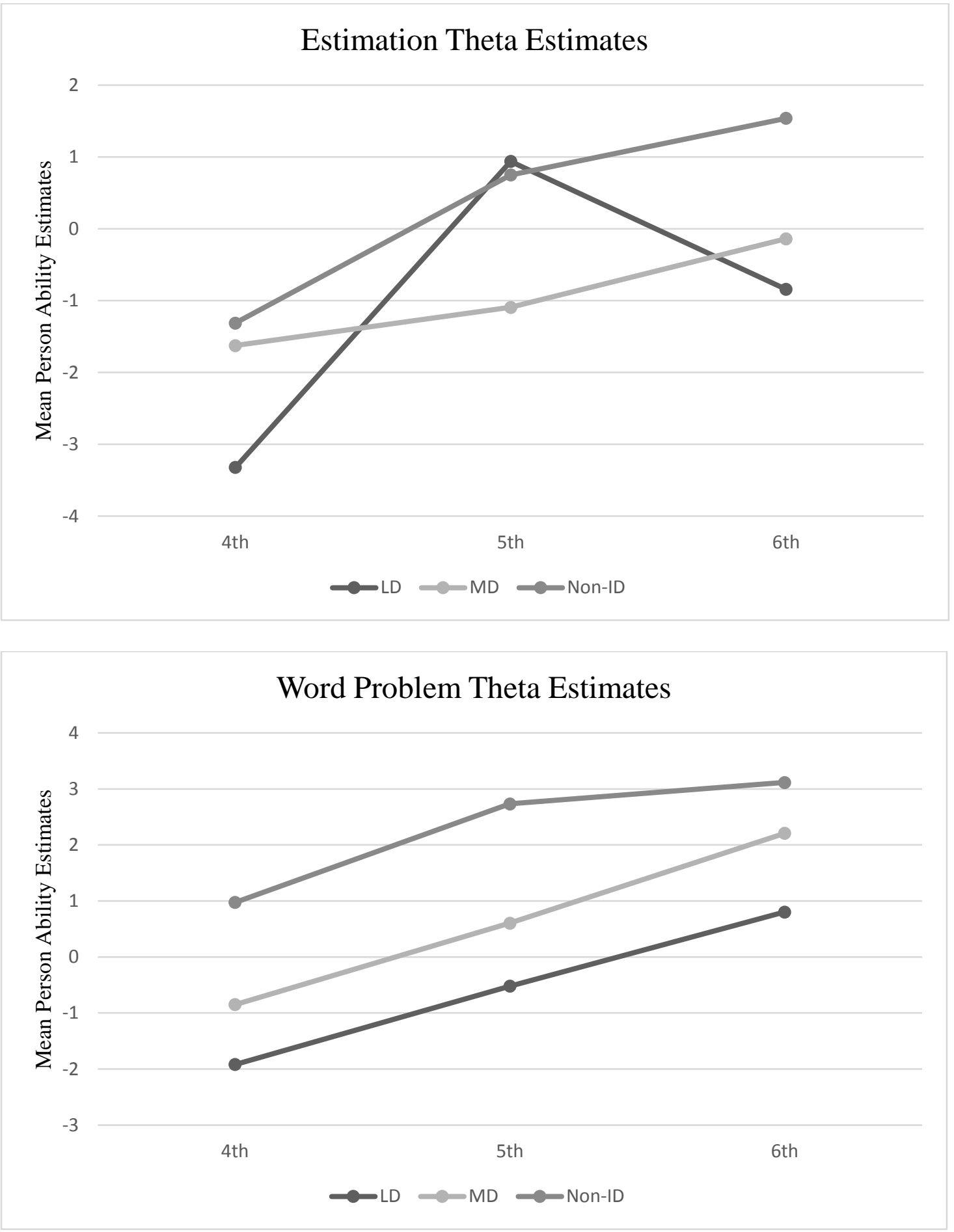

Ho: The influence of each domain-general and domain-specific factor will vary as a function of student ability group and grade level. 
This hypothesis was created to examine if the impact of each predictor variable was stable across participants of differing ability levels. For example, perhaps attention is not particularly important for a typically achieving student, but is extremely important for a student with LD. As math achievement scores were kept continuous in the path analysis model there was no way to examine whether the influence of certain factors varied for students of different ability groups. To analyze this question a categorical variable grouping students as non-identified, MD (bottom $30 \%$ on the WRAT-4), and LD (bottom $30 \%$ on the WRAT-4 and had school identified disabilities) was created. A series of ANOVAs were run which examined the interaction effect between each predictor variable and ability group status on the various fraction outcomes of interest.

There were not significant interactions between attention and ability group on the conceptual understanding of fractions, $F(19,152)=1.315, p=.959, \eta^{2}=.058$; on fraction estimation, $F(19,153)=1.047, p=.412, \eta^{2}=.115$; or on word problem set-up, $F(19,153)=$ $.691, p=.824, \eta^{2}=.079$. Overall these results indicate that the impact of attention on fraction outcomes is relatively stable across students of different ability levels. There was one interaction of note. Though not significant after correcting for family wise error rate there is a potential interaction between attention and ability group for fraction calculation, $F(19,153)=1.712, p=$ $.039, \eta^{2}=.175$. To explore this relationship the data file was split by ability grouping and a regression analysis was run for attention on fraction computation. Next, the researcher plotted the regression lines for each group to create Figure 8. It appears as if students with disabilities do not benefit nearly as much from higher levels of attention than their non-identified peers. This relationship should be explored in future studies to see if there is any significance to the potential interaction. 
Figure 8

Ability Group by Attention Interaction Effects on Computation

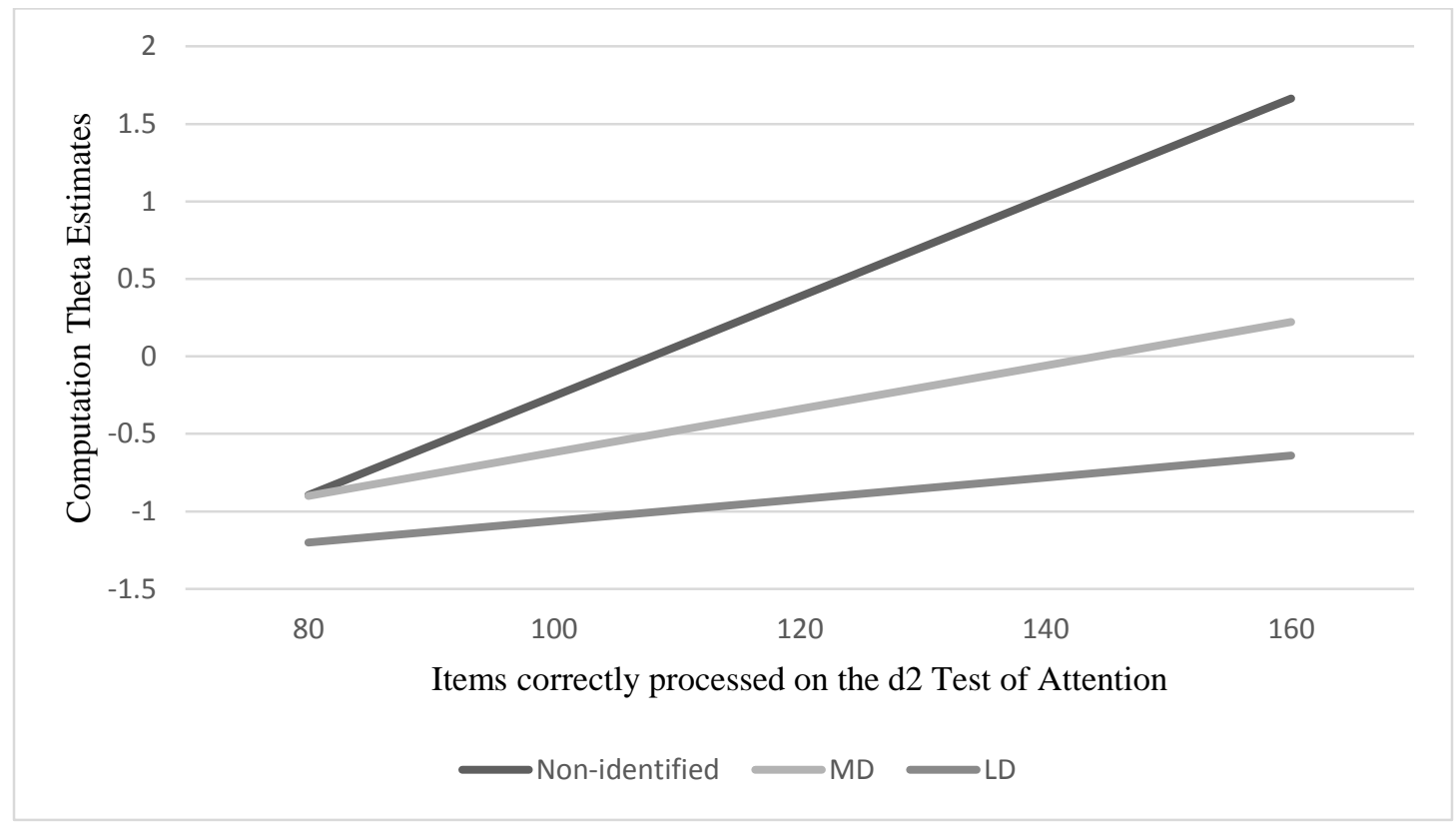

I next examined the interaction between nonverbal reasoning and ability level. There was no significant interaction between nonverbal reasoning and ability level for the conceptual understanding of fractions, $F(17,259)=.389, p=.987, \eta^{2}=.025 ;$ fraction calculation, $F(17$, $259)=.878, p=.603, \eta^{2}=054$; fraction estimation, $F(17,259)=.741, p=.759, \eta^{2}=.046$; or word problem set-up, $F(17,259)=.976, p=.486, \eta^{2}=.060$. The influence of nonverbal reasoning was stable for students in different ability groups.

Lastly, the researcher examined the interaction between the conceptual understanding of fractions and participant ability group on fraction calculation, $F(20,188)=.786, p=.728, \eta^{2}=$ .077 ; fraction estimation, $F(20,188)=.992, p=.474, \eta^{2}=.095$; and word problem set-up, $F(20$, $188)=1.437, p=.110, \eta^{2}=.133$. There was no significant interaction between ability group and the conceptual understanding of fractions on the fraction outcomes of participants.

Ho: The d2 Test of Attention and results from the cooperation subscale of the SSRS each explain unique amounts of variance in fraction outcomes (i.e., they are not measuring the 


\section{same thing).}

Teacher ratings of student attention have been used as a proxy for student classroom attention (e.g., Fuchs et al., 2006, 2008; Hecht \& Vagi, 2010), and have been found to be strongly linked to various fraction outcomes. The question has been raised as to whether the cooperation subscale of the SSRS (used in several of the cited studies) is actually capturing attention. This hypothesis was tested by collecting SSRS teacher ratings and a more direct measure of student attention (i.e., d2 Test of Attention). Exploratory analysis revealed that both measures were significantly linked to various fraction outcomes, and that perhaps SSRS scores were slightly more strongly associated with fraction outcomes. To determine if the SSRS and d2 Test of Attention were explaining the same variance or different variance the researcher decided to examine the partial and semi-partial correlations between the two measures of attention and the variables they were predicting.

A $\mathrm{R}^{2}$ score indicates what percentage of variance in a certain variable is explained by another variable. A partial correlation is the correlation that remains between two variables when all of the variance also explained by a third variable is removed from the first IV and the DV. A semi-partial correlation is the correlation that remains between an IV and DV after controlling for the influence of a covariate on either the IV or DV but not both. If partial and semi-partial correlations drop significantly from the original $\mathrm{R}^{2}$ correlation it indicates that the two predictor variables are explaining the same variance. Table 3 reports the $\mathrm{R} 2$, partial, and semi-partial correlations for each fraction outcome.

Examination of the partial and semi-partial correlations indicate that each measure of attention explains significant amounts of unique variance in the dependent variables. There is some overlap as seen by the average 0.1 drop in correlation from the $\mathrm{R}^{2}$ correlation to the semi- 
partial correlation. In spite of some overlap, these data lead me to conclude that the SSRS and d2 Test of Attention are not capturing the same aspects of attention, or that the SSRS is capturing some other phenomenon altogether.

Table 3

Partial and Semi-Partial Attention Correlations

\begin{tabular}{lccc}
\hline & R2 & Partial & Semi-partial \\
\hline SSRS - Conceptual & 0.497 & 0.427 & 0.389 \\
d2 - Conceptual & 0.406 & 0.316 & 0.301 \\
& & & \\
SSRS - Calculate & 0.483 & 0.411 & 0.381 \\
d2 - Calculate & 0.372 & 0.269 & 0.236 \\
& & & \\
SSRS - Estimate & 0.387 & 0.32 & 0.303 \\
d2 - Estimate & 0.315 & 0.225 & 0.303 \\
& & & 0.371 \\
SSRS - WP set-up & 0.461 & 0.396 & 0.214 \\
d2 - WP set-up & 0.352 & 0.242 & \\
\hline
\end{tabular}




\section{DISCUSSION}

The purposes of this study were to evaluate the relationships among student attention, nonverbal reasoning, math achievement, and a variety of fraction outcomes, and to examine how those relationships vary for students of differing ability levels across grade levels. The following sections will discuss some empirical support for the conceptual model used in this study, examine the influence of each predictor variable, examine fraction outcomes, and then describe the general implications of the results.

\section{Conceptual Understanding of Fractions}

One factor that has consistently been suggested as a predictor of overall fraction achievement is the conceptual understanding of fractions (e.g., Hecht, 1998; Hecht et al., 2003). Although there is evidence that procedural skills influence conceptual understanding (Geary, 1993; Pantziara \& Philippou, 2012; Rittle-Johnson \& Siegler, 1998), the path analysis model placed the conceptual understanding of fractions as a predictor of procedural fraction outcomes (i.e., fraction computation). That is, in contrast to researchers who assert that procedural ability drives fraction understanding, the path analysis model gave conceptual understanding a more powerful role in than procedural skills. Many reports support the view that the direction of the relationship is primarily from conceptual understanding to procedural outcomes (e.g., Goldman \& Hasselbring, 1997; Hecht et al., 2003). This is not to say that procedural skills cannot translate to increased conceptual understanding, just that the relationship seems to be stronger moving in the other direction (Hecht \& Vagi, 2010). Findings from this study support the idea that the conceptual understanding of fractions is a significant predictor of other fraction outcomes. These relationships were particularly strong as the standardized regression weights from conceptual understanding to fraction computation, fraction estimation, and word problem set-up were larger 
than any other relationships in the model.

Interpretation of the strong relationship found in this study between cognitive factors (i.e., attention and nonverbal reasoning) and the conceptual understanding of fractions, and between the conceptual understanding of fractions and other fraction outcomes should be applied only to the skills assessed in this study. This means that results from this study did not assess all possible fraction skills; for example, this study did not assess any practical application of fraction knowledge to real life problem solving.

This study corroborates the findings of previous research (Hecht, 1998; Hecht et al., 2003; Hecht \& Vagi, 2010) and provides further evidence that the conceptual understanding of fractions is highly important for overall fraction mastery. This study extends the findings because the influence of the conceptual understanding of fractions was observed on a different population of students with a different set of measures. Not only were the standardized regression weights for all three fraction outcomes large, some of the influence of nonverbal reasoning and attention was mediated through the conceptual understanding of fractions.

Future research should examine the influence of specific fraction concepts (e.g., value of fractions, equivalency) on overall fraction skills, and test the theory that these are core deficits by designing and implementing interventions targeting these skills.

\section{Nonverbal Reasoning}

Among the various potential core deficits that could have been investigated this study focused on nonverbal reasoning for two reasons. First, nonverbal reasoning theoretically assesses a person's ability to see patterns, identify differences, and generally work with shapes and representations of objects. As fractions are often represented tangibly with manipulatives and pictorially, it would stand to reason that strong nonverbal reasoning skills would be linked to an 
understanding of fractions. Second, Seethaler et al. (2011) discussed the fact that nonverbal reasoning has been shown to be significantly linked to whole number word problem solving but not to calculation skills. Seethaler et al. ultimately found that nonverbal reasoning was significantly linked to fraction calculation, but they did not assess any other fraction skills. The idea that a cognitive factor could explain certain aspects of fraction skill development but not others was something that needed to be explored further.

Results from this study lend support to the influence of nonverbal reasoning on the fraction outcomes of students. Interestingly, the only path in the model that was not significant was between nonverbal reasoning and fraction computation (see Table 2). Standardized regression weights for the indirect effects of nonverbal reasoning on fraction calculation indicate that the influence of nonverbal reasoning is mediated through the conceptual understanding of fractions. Logically this weak relationship makes sense. Nonverbal reasoning theoretically has more to do with problem solving and critical thinking than applying procedural algorithms correctly.

Findings from this study confirm and extend the thought that nonverbal reasoning is significantly related to fraction outcomes. This is the first study that assessed nonverbal reasoning's relationship to conceptual understanding of fractions, setting up word problems involving fractions, and estimating fraction. All of these relationships were significant and effect sizes were moderate, indicating that nonverbal reasoning is a significant factor in overall fraction performance.

This study's findings seem to conflict with the results of Seethaler et al. (2011) who found that nonverbal reasoning was significantly related to fraction calculation. This conflict may be explained by this study's inclusion of the conceptual understanding of fractions as a 
mediating variable, whereas Seethaler et al. simply assessed fraction calculation. As this study found the indirect effects of nonverbal reasoning on fraction calculation were much larger than the direct effects, it stands to reason that the non-significant findings from this study may be due to the fact that the influence of nonverbal reasoning was wholly mediated through conceptual understanding.

Future research should explore what aspects of the conceptual understanding of fractions are influenced by nonverbal reasoning. Also, further research should include nonverbal reasoning in a longitudinal study to determine its influence on the growth of fraction skills.

\section{Attention}

This study examined attention as a core deficit that might impact fraction outcomes because both theory and evidence support that assumption (Hecht et al., 2003; Hecht \& Vagi, 2010). It is not a large leap to imagine how a student's inability to focus on important information and to sustain attention could detrimentally influence any academic outcome. On top of the face-value, common sense perspective about it, the idea that attention, or something close to it has a large influence on fraction outcomes is supported by previous research. Fuchs et al., (2006) found that teacher ratings of attentive behavior were significantly correlated with math computation and word-problem solving skills. Hecht et al., (2003) and Hecht and Vagi (2010) found that in addition to predicting initial fraction achievement, attentive classroom behavior mediated a large portion of the growth in student fraction skills over time.

Previous studies examining the relationship between attention and math performance have employed different measures of attention. Hecht and Vagi (2010) called for more research about the specific aspects of student attention that were captured by teacher ratings, but there are other measures of attention, as well, including the measures used in this study. Fuchs et al. 
(2006) more directly addressed the problem of identifying what is being measured by teacher ratings of student attention by proposing three possibilities. Does students' ability to sustain their attention in the face of distraction creates the opportunity to engage with academic activities in the classroom and give them a greater chance to succeed? Alternatively, students with poor math skills may lose focus during math activities due to their difficulty with the subject. This possibility explains the observed strong relationship between teacher ratings of attention and math outcomes but flips the directionality of the relationship. Yet another possibility is that teacher ratings of student attention are clouded by overall academic achievement and are not actually assessing attention accurately. Another possibility is that students may attend to aspects of the instructional activities that are not as relevant for core concepts about fractions as those that are critical, cuing off irrelevant aspects of displays (e.g., the number of lines drawn in dividing a figure rather than the number of segments the lines define (e.g., Kelly, Gerston, \& Carine, 1980).

Makers of the $\mathrm{d} 2$ Test of Attention claim their test assesses selective attention and sustained attention. Selective attention is the ability to focus on one or two important stimuli, while suppressing awareness of competing distractions (Brickenkamp \& Zillmer, 1998). Sustained attention refers to the ability to maintain focus and productivity at a sustained level of accuracy over a period of time. Results from this study confirm that attention in the classroom is related to all fraction outcomes, although the relationships do not appear to be as robust as when assessed by teacher ratings in previous studies (e.g., Hecht \& Vagi, 2010). It is interesting to note that attention's relationship to fraction calculation was stronger than its relationship to estimation or word problem solving. This makes intuitive sense as the ability to focus on detail and follow a set of procedures requires the ability to focus and sustain attention. 
The cooperation subscale of the SSRS was also significantly related to fraction outcomes, but, as seen in the results section, explained very little of the same variance as the $\mathrm{d} 2$ Test of Attention. There are a few possible explanations for the two supposed measures of attention not capturing the same variance. Perhaps teacher ratings are just capturing different aspects of attention not assessed by the $\mathrm{d} 2$ Test of Attention? Another possibility is that teacher ratings of attention are capturing something more along the lines of behavioral inhibition than attention. Slips in productivity or accuracy are captured on the $\mathrm{d} 2$ indicating that the student may have been distracted or lost focus at some point. These minor slips in attention would not be captured by teacher ratings; only incidents that escalate to the point of some physical response may be observed by a teacher. A third alternative is that Fuchs et al., (2006) was correct and that teacher ratings of attention merely serve as a proxy for student achievement.

Although this study lends support to the idea that teacher ratings of student attention may not actually be capturing attention, there is not enough evidence to make that claim definitively. More research needs to be conducted to confirm more direct measures of attention's influence on fraction outcomes and to determine what exactly is being captured by teacher ratings of student attention.

\section{Amount of Variance Explained by the Model}

Analysis of the reported squared multiple correlations given in Figure 9 in the appendix section indicate that the included variables explain large amounts of variance in the conceptual understanding of fractions (45\%), fraction calculation (40\%), fraction estimation (31\%), and word problem set-up (39\%). These numbers indicate that attention and nonverbal reasoning along with previous math achievement explain almost half of the variance in the conceptual understanding of fractions observed in this study. It is important to note the amount of variance 
explained by the model to understand that the included variables are only part of what explains overall fraction performance. Any complete theory of fraction performance would include more intrinsic (e.g., working memory), and extrinsic factors (e.g., classroom environment, method of fraction engagement). These other factors need to be explored in future research to better understand their respective influence on fraction achievement and how they interact with each other.

\section{Ability Group Differences}

A particular motivation of this study was to examine the influence of different cognitive factors on the fraction outcomes of students across different ability levels, and to examine what specific deficits students with MD or LD exhibited. Fairly obviously, as seen in the results section, students with LD and students with MD performed worse than their non-identified peers on nearly every measure.

One interesting result from this study came from the manner in which students were placed in their respective ability groups. Any student scoring below the $30^{\text {th }}$ percentile was eligible to be in the MD group, and those scoring below the $30^{\text {th }}$ percentile and having a school identified disability were placed in the LD group. As a result of this, the mean WRAT-4 score for the MD group (M=90.41) and the LD group (M=89.40) were practically identical. This allows for a comparison of fraction ability between the MD and LD groups while having a rough control for overall math achievement. Essentially, what differences in fraction performance can be attributed to the student having an IEP? Examination of Figure 6 shows a visual representation of person ability estimates by ability grouping. Participants grouped in the LD group scored significantly lower on the conceptual assessment than their peers in the MD group. As this study and previous studies have demonstrated the significance of the conceptual 
understanding of fractions it is worrisome that students with IEPs struggle even more than their math achievement scores would predict.

Similar significant post hoc comparisons between the LD and MD groups were found for word problem set-up, and nonverbal reasoning. It is interesting to note that students with school identified disabilities (LD) scored significantly lower than their peers with MD on the nonverbal reasoning assessment but had very similar scores on the math achievement assessment. Perhaps, as the WRAT-4 is largely calculation based, it does not pick up on conceptual and reasoning deficits that students with disabilities might have? This study provides evidence that nonverbal reasoning is a specific deficit area for students with LD that negatively influences their overall fraction performance.

Another area of interest for students with disabilities examined in this study was whether the gap in fraction ability widened between the fourth and sixth grades. Although this study's hypothesis was that the differences between those with and without disabilities would be larger for students in higher grades, the evidence did not support this hypothesis. This is an interesting result as students in the LD group were already significantly behind their non-identified peers in the fourth grade. How did that gap develop if there appears to be no widening of the gap between the fourth and sixth grades? At present there is no answer to this question and would actually predict that a more targeted study with a greater representation of students with disabilities would not replicate these findings.

This study provides evidence that nonverbal reasoning, attention, and the conceptual fraction knowledge each uniquely predict observed variation in fraction performance across ability levels. Findings from this study indicate that the influence of attention, nonverbal reasoning, and the conceptual understanding of fractions remains steady for students classified in 
the non-identified, MD, and LD groups. Although most interactions were non-significant, there was a potential interaction between attention and ability group on fraction calculation. It is important to note again that the interaction was no longer significant after correcting for family wise error rate. Examination of Figure 8 indicates that, for students with disabilities, there is some factor that suppresses the benefits students without disabilities receive in fraction calculation from increasing levels of attention. Hecht and Vagi (2010) found that following students longitudinally provided a different picture of the factors that influence fraction performance in comparison to utilizing a cross-sectional research design. Specifically they found that teacher ratings of student attention explained a large portion of observed differences in fraction ability growth. Another study should be conducted with a direct measure of attention and a nonverbal reasoning assessment that captures student fraction knowledge growth over time.

\section{Implications for Practice}

The importance of conceptual fraction knowledge and the relative deficit that students with disabilities have in that area will, hopefully, lead to interventions targeted at developing mastery of some of the underpinning concepts of fractions. Although this study descriptively provides evidence for a relationship between the conceptual factors and other fraction outcomes, an intervention that teaches core fraction concepts can provide causal evidence of a relationship if it exists.

Although of the three fraction predictors, attention was most strongly linked to fraction calculation, an interesting finding from this study is that nonverbal reasoning did not have a significant relationship to fraction calculation. These results lend support to the idea that fractions are not a monolithic concept and that unique combinations of student abilities (e.g., 
attention, nonverbal reasoning, working memory) will lead to unique patterns of fraction performance. By identifying common student error patterns across the fourth through sixth grades, this study lends support to the idea that although there is improvement over time, many students continue to struggle with basic fraction skills into middle school. Although the errors student made and concepts assessed in this study in no way represent a complete knowledge of fractions, results provide a clear picture of students struggling to understand and apply the various concepts associated with fraction skill development (e.g., reducing, number lines, equivalent fractions, fractions must be divided into equal portions).

\section{Limitations}

Several limitations should be taken into consideration when interpreting the results of this study. The first limitation is the limited scope of the current study, both in what types or rational numbers and fraction skills were not assessed and in the fact that other significant cognitive factors (e.g., working memory, visual-spatial skills) were not included in this study. Although the types of fraction questions in this study were limited to those participants in the fourth grade would be familiar with, there are more advanced and applied fraction problems (e.g., fractions as operators or quotients) that should be included in future research, for example, this study did not assess any practical application of fraction knowledge to real life problem solving. Also, there is little current evidence as to what cognitive factors influence other rational number skills (e.g., decimals, percents).

A second limitation is one shared with Hecht and Vagi (2010) and concerns the fact that this study in no way captured the nature of classroom instruction, and how that might moderate the influence of cognitive factors on fraction outcomes. Perhaps in a strong and engaging teacher's classroom those with attention deficits will be less affected than students in a non- 
engaging teacher's classroom. Due to the manner in which the participating schools were set up, only three teachers were represented in this study, one for each grade level. Consequently it would be impossible to separate teacher effects on fraction performance from grade level effects on fraction performance. It is not a stretch to assume that quality teacher will lead to higher student fraction outcomes, but future research should investigate the interaction between teacher quality and the influence of cognitive factors on fraction outcomes. This study examined the influence of internal factors (i.e., nonverbal reasoning, attention), but any true understanding of fraction skill growth needs to account for external factors as well (e.g., teacher, methods of instruction).

A third limitation is that the population of students recruited to participate in this study scored extremely high on the WRAT-4 computation subtest. The mean score across all three grade levels was around 110. These highly unusual results limited the number of participants who fell more than a standard deviation below the mean, and therefore limited the number of participants included in the MD, and LD groupings.

A fourth limitation is the generalizability of the results from this study. Fourth and fifth grade participants were drawn from a rural county in Virginia while sixth grade students were drawn from a large private school. These populations of students need to be kept in mind when interpreting the results of this study. Future studies should seek to include students from urban and suburban settings, as well as students in the seventh and eighth grades.

\section{Conclusion}

In conclusion, the findings suggest that domain general and domain specific variables significantly and uniquely contribute to different types of fraction knowledge, even after controlling for general math ability. This study provides initial evidence of the influence of 
nonverbal reasoning on conceptual understanding, estimation, and word problem set-up of fraction problems, and provides evidence that nonverbal reasoning may not be significantly linked to fraction calculation. This study also found that a more direct measure of student attention was significantly linked to fraction outcomes, though the relationship was not as strong as those found in previous studies using teacher ratings of attention (Hecht et al., 2003; Hecht \& Vagi, 2010). Furthermore, teacher ratings of student attention and results of the $\mathrm{d} 2$ Test of Attention explained very little of the same variance on fraction outcomes. The influence of cognitive factors seemed to hold steady across participants in all three ability groups. Participants with school identified disabilities showed significant deficits in the conceptual understanding of fractions even when controlling for general math ability. Essentially, students with LD separated themselves from those with MD in their weak conceptual understanding of fractions. Students with LD also scored significantly below their MD peers in nonverbal reasoning and word problem set-up. Due to the observed high level of influence that the conceptual understanding of fractions has on other fraction outcomes, students with LD are particularly at risk for fraction failure. 


\section{REFERENCES}

Algozzine, B., O’Shea, D. J., Crews, W. B., \& Stoddard, K. (1987). Analysis of mathematics competence of learning disabled adolescents. The Journal of Special Education, 21, $97-$ 107.

Alloway, T. P., \& Passolunghi, M. C. (2011). The relationship between working memory, IQ, and mathematical skills in children. Learning and Individual Differences, 21, 133-137.

Amit, M., \& Fried, M. N. (2002). High-stakes assessment as a tool for promoting mathematical literacy and the democratization of mathematics education. Journal of Mathematical Behavior, 21, 499-514.

Andersson, U. (2008). Mathematical competencies in children with different types of learning difficulties. Journal of Educational Psychology, 100, 48-66. doi:10.1037/0022-0663. 100.1.48

Andersson, U., \& Lyxell, B. (2007). Working memory deficit in children with mathematical difficulties: A general or specific deficit? Journal of Experimental Child Psychology, 96, 197-228. doi: 10.1016/j.jecp.2006.10.001

Andrich, D. (2004). Controversy and the Rasch model: a characteristic of incompatible paradigms? Medical Care, 42, 1-16.

Baddeley, A. (2000). The episodic buffer: A new component of working memory? Trends in Cognitive Sciences, 4, 417-423.

Ball, D. L. (1990). The mathematical understandings that prospective teachers bring to teacher education. The Elementary School Journal, 90, 449-466.

Barbaresi, W. J., Katusic, S. K., Collagin, R. C., Weaver, A. L., \& Jacobsen, S. J. (2005). Math learning disorder: Incidence in a population-based birth cohort, 1976-82, Ambulatory 
Pediatrics, 5, 281-289. doi:10.1007/s10803-008-0645-8

Barker, T. A., Torgesen, J. K., \& Wagner, R. K. (1992). The role of orthographic processing skills on five different reading tasks. Reading Research Quarterly, 27, 334-345.

Barkley, R. A., Fischer, M., Edelbrock, C. S., \& Smallish, L. (1990). The adolescent outcome of hyperactive children diagnosed by research criteria: I. An 8-year prospective follow-up study. Journal of the American Academy of Child and Adolescent Psychiatry, 29, 546557.

Bates, M. E., \& Lemay, E. P., Jr. (2004). The d2 test of attention: Construct validity and extensions in scoring techniques. Journal of the International Neuropsychological Society, 10, 392-400.

Behr, M., Harel, G., Post, T. \& Lesh, R. (1993). Rational Numbers: Toward a Semantic Analysis-Emphasis on the Operator Construct. In T. P. Carpenter, E. Fennema, \& T.A. Romberg, (Eds.), Rational Numbers: An Integration of Research (pp. 13-47). NJ: Lawrence Erlbaum

Bennett, R. E., Gottesman, R. L., Rock, D. A., \& Cerullo, F. (1993). Influence of behavior perceptions and gender on teachers' judgments of students' academic skill. Journal of Educational Psychology, 85, 347-356.

Berg, D. H., \& Hutchinson, N. L. (2010). Cognitive processes that account for mental addition fluency differences between children typically achieving in arithmetic and children atrisk for failure in arithmetic. Learning Disabilities: A Contemporary Journal, 8, 1-20.

Blair, C., \& Razza, R. P. (2007). Relating effortful control, executive function, and false believe understanding to emerging math and literacy ability in kindergarten. Child Development, $78,647-663$ 
Bottge, B. A. (1999). Effects of contextualized math instruction on problem solving of average and below-average achieving students. Journal of Special Education, 33(2), 81-92.

Bottge, B. A., \& Hasselbring, T. S. (1993). A comparison of two approaches for teaching complex, authentic mathematics problems to adolescents in remedial math classes. Exceptional Children, 59, 556-511.

Bottge, B. A., Heinrichs, M., Mehta, Z.D., \& Hung, Y. H. (2002).Weighing the benefits of anchored math instruction for students with disabilities in general education classes. Journal of Special Education, 35(4), 186-200.

Bottge, B. A., Rueda, E., Grant, T. S., Stephens, A. C., Laroque, P. T. (2010). Anchoring problem-solving and computation instruction in context-rich learning environments. Exceptional Children, 76, 417-437.

Botwinick, M. M., Braver, T. S., Barch, D. M., Carter, C. S., \& Cohen, J. D. (2001). Conflict monitoring and cognitive control Psychological Review, 108, 624-652

Brickenkamp, R., \& Zillmer, E. (1998). The d2 Test of Attention. Seattle, Washington: Hogrefe \& Huber Publishers.

Browder, D. M., Jimenez, B. A., Spooner, F., Saunders, A., Hudson, M., \& Bethune, K. S. (2012). Early numeracy instruction for students with moderate and severe developmental disabilities. Research \& Practice for Persons with Severe Disabilities, 37, 308-320.

Brown, R. T., \& Borden, K. A. (1986). Hyperactivity at adolescence: Some misconceptions and new directions. Journal of Clinical Child Psychology, 15, 194-209.

Brown, M., Hart, K., \& Kucherman, D. (1984). Chelsea Diagnostic Mathematics Tests. Fractions 1. Incorporating Fractions 1 (Computation). Windsor, England: NFERNelson. 
Bull, R., Espy, K. A., \& Wiebe, S. A. (2008). Short-term memory, working memory, and executive functioning in preschoolers: Longitudinal predictors of mathematical achievement age 7 years. Developmental Neuropsychology, 33, 205-228.

Bull, R., \& Scerif, G. (2001). Executive functioning as a predictor of children's mathematical ability: Inhibition, switching, and working memory. Developmental Neuropsychology, 19, 273-293.

Butler, F. M., Miller, S. P., Crehan, K., Babbitt, B. \& Pierce, T. (2003). Fraction instruction for students with mathematics disabilities: Comparing two teaching sequences. Learning Disabilities Research \& Practice, 18, 99-111

Bryant, D. P., Bryant, B. T., \& Hammill, D. D. (2000). Characteristics behaviors of students with LD who have teacher-identified math weaknesses. Journal of Learning Disabilities, 33, 168-177-199.

Byrnes, J. P., \& Wasik, B. A. (1991). Role of conceptual knowledge in mathematical procedural learning. Developmental Psychology, 27, 777-786.

Calhoon, M. B., Emerson, R. W., Flores, M. W., \& Houchins, D. E. (2007). Computational fluency performance profile of high school students with mathematics disabilities. Remedial and Special Education, 28, 292-303.

Cawley, J. F., \& Miller, J. H. (1989). Cross-sectional comparisons of the mathematical performance of children with learning disabilities: Are we on the right track toward comprehensive programming? Journal of Learning Disabilities, 22, 250-254.

Cawley, J. F., Parmar, R. S., Lucas-Fusco, L. M., Kilian, J. D., \& Foley, T. E. (2007). Place value and mathematics for students with mild disabilities: Data and suggested practices. Learning Disabilities: A contemporary Journal, 5, 21-39. 
Caverni, J-P., Fabre, J-M., \& Gonzalez, M. (1990). Cognitive biases: Their contribution for understanding human cognitive processes. In J-P. Caverni, J-M. Fabre, \& M. Gonzales (Eds.), Cognitive biases (pp. 7-12). Amsterdam, The Netherlands: North-Holland.

Charalambous, C., \& Pitta-Pantazi, D. (2007). Drawing on a theoretical model to study students' understandings of fractions. Educational Studies in Mathematics, 64, 293-316.

Chen, X., Rubin, K. H., \& Li, D. (1997). Relation between academic achievement and social adjustment: Evidence from Chinese children. Developmental Psychology, 33, 518-525.

Cirino, P. T., Fletcher, J. M., Ewing-Cobbs, L., Barnes, M. A., \& Fuchs, L. S. (2007). Cognitive arithmetic differences in learning difficulty groups and the role of behavioral inattention. Learning Disabilities Research \& Practice, 22, 25-35.

Cohen, J., Cohen, P., West, S. G., \& Aiken, L. S. (2003). Applied multiple regression/correlation analysis for the behavioral sciences. 3rd ed. Mahwah, NJ: Erlbaum.

Common Core State Standards. (2013) retrieved on 11/05/2013 from www.corestandards.org.

Copeland, A. P., \& Wisniewski, N. M. (1981). Learning disability and hyperactivity: Deficits in selective attention. Journal of Experimental Child Psychology, 32, 88-101.

Creswell, J. W. (2008) Educational research: Planning, conducting, and evaluating quantitative and qualitative research (3rd ed.). Upper Saddle River, NJ; Pearson

De Smedt, B., \& Boets, B. (2010). Phonological processing and arithmetic fact retrieval: Evidence from developmental dyslexia. Neuropsychologia, 48, 3973-3981. doi:10.1016/jneuropsychologia.2010.10.018

Fan, J. F., McCandliss, B. D., Sommer, T., Raz, A., \& Posner, M. I. (2002). Testing the efficiency and independence of attentional networks. Journal of Cognitive Neuroscience, $14,340-347$. 
Feldon, D. F. (2007). The implications of research on expertise for curriculum and pedagogy. Educational Psychology Review, 19, 91-110.

Flores, M. M., \& Kaylor, M. (2007). The effects of a direct instruction program on the fraction performance of middle school students at-risk for failure in mathematics. Journal of Instructional Psychology, 34, 84-94

Fuchs, L. S., Fuchs D., Compton D. L., Powell S. R., Seethaler P. M., Capizzi A. M., et al. (2006). The cognitive correlates of third-grade skill in arithmetic, algorithmic computation, and arithmetic word problems. Journal of Educational Psychology. 98, 2943.

Fuchs, L. S., Fuchs D., Steubing K., Fletcher J. M., Hamlett C. L., Lambert W. (2008). Problem solving and computational skill: Are they shared or distinct aspects of mathematical cognition? Journal of Educational Psychology. 100, 30-47.

Fuchs, L. S., Powell, S. R., Seethaler, P. M., Cirino, P. T., Fletcher, J. M., Fuchs, D., \& Hamlett, C. L. (2010). The effects of strategic counting instruction, with and without deliberate practice, on number combination skill among students with mathematics difficulties. Learning and Individual Differences, 20, 89-100. doi:10.1016/j.lindif.2009.09.003

Gallistel, C. R., \& Gelman, R. (2000). Non-verbal numerical cognition: from reals to integers. Trends in Cognitive Sciences, 4, 59-65.

Geary, D. C. (1993) Mathematical disabilities: Cognitive, neuropsychological, and genetic components. Psychological Bulletin, 114, 345-362.

Geary, D. C. (1994). Children's mathematical development: Research and practical applications. Washington, DC: American Psychological Association.

Geary, D. C. (2004). Mathematics and learning disabilities. Journal of Learning Disabilities, 37, 
4-15. doi:10.1177/00222194040370010201

Geary, D. C. (2007). An evolutionary perspective on learning disability in mathematics. Developmental Neuropsychology, 32, 471-519.

Geary, D. C., Hoard, M. K., Byrd-Craven, J., Nugent, L., Numtee, C. (2007). Cognitive mechanisms underlying achievement deficits in children with mathematical learning disability. Child Development,78, 1343-1359

Gilmore, C. K., McCarthy, S. E., \& Spelke, E. S. (2010). Non-symbolic arithmetic abilities and mathematics achievement in the first year of schooling. Cognition, 115, 394-406. doi:10.1016/j.cognition.2010.02.002

Goldman, S. R., \& Hasselbring, T. S. (1997). Achieving meaningful mathematics literacy for students with LD. Journal of Learning Disabilities, 30, 198-208.

Gresham, F. M., \& Elliott, S. N. (1990). Social skills rating system. Circle Pines, MN.

Grobecker, B. (1999). The evolution of proportional structures in children with and without learning differences. Learning Disability Quarterly, 22, 192-211

Hagen, J. W. (1967). The effect of distraction on selective attention. Child Development, 38, 685-694.

Hallahan, D. P., Gajar, A. H., Cohen, S. B., \& Tarver, S. G. (1978). Selective Attention and Locus of Control in Learning Disabled and Normal Children. Journal of Learning Disabilities, 11, 47-52.

Hallahan, D. P., Kauffman, J. M., \& Ball, D. W. (1973). Selective attention and cognitive tempo of low achieving and high achieving sixth-grade males. Perceptual Motor Skills, 36,579583.

Hallet, D., Nunes, T., \& Bryant, P. (2010). Individual differences in conceptual and procedural 
knowledge when learning fractions. Journal of Educational Psychology, 102, 395-406. doi:10.1037/a0017486

Hanich, L. B., Jordan, N. C., Kaplan, D., \& Dick, J. (2001). Performance across different areas of mathematical cognition in children with learning disabilities. Journal of Educational Psychology, 93, 615-626. doi:10.1037//0022-0663.93.3.615

Hambleton, R. K., Swaminathan, H., \& Rogers, H. J. (1991). MMSS: Fundamentals of item response theory. Newbury Park, CA: Sage.

Hammond, J. L., Hirt, M., \& Hall, S. S. (2012). Effects of computerized match-to-sample training on emergent fraction-decimal relations in individuals with fragile $\mathrm{x}$ syndrome. Research in Developmental Disabilities, 33, 1-11.

Hecht, S. A. (1998). Toward an information-processing account of individual differences in fraction skills. Journal of Educational Psychology, 90, 545-559.

Hecht, S. A., Close, L., \& Santisi, M. (2003). Sources of individual differences in fraction skills. Journal of Experimental Child Psychology, 86, 277-302.

Hecht, S. A., \& Vagi, K. J. (2010). Sources of group and individual differences in emerging fraction skills. Journal of Educational Psychology, 102, 843-859. doi: 0022$0663 / 10 / \$ 12.00$

Hecht, S. A., \& Vagi, K. J. (2012). Patterns of strengths and weaknesses in children's knowledge about fractions. Journal of Experimental Psychology, 111, 212-229. doi:10.1016/j.jecp.2011.08.012

Hecht, S. A., Vagi, K. J., \& Torgesen, J. K. (2007). Fraction skills and proportional reasoning. In D. B. Berch \& M. M. M. Mazzocco (Eds.), Why is math so hard for some children? The nature and origins of mathematical learning difficulties and disabilities (pp. 121-132). 
Baltimore, MD: Brookes.

Hibbard, J. H., Peters, E., Dixon, A., \& Tusler, M. (2007). Consumer competencies and use of comparative quality information: It isn't just about literacy. Medical Care Research and Review, 64, 379-394. doi:10.1177/1077558707301630

Holmes, J., \& Adams, J. W. (2006). Working memory and children's mathematical skills: Implications for mathematical development and mathematics curricula. Educational Psychology, 26, 339-366. doi:10.1080/01443410500341056

Ivrendi, A. (2011). Influence of self-regulation on the development of children's number sense. Early Childhood Education Journal, 39, 239-247.

Joseph, L. M., \& Hunter, A. D. (2001). Differential application of a cue card strategy for solving fraction problems: Exploring instructional utility of the cognitive assessment system. Child Study Journal, 31, 123-136.

Jordan, N. C, \& Hanich L. B. (2003). Characteristics of children with moderate mathematics deficiencies: A longitudinal perspective. Learning Disabilities Research and Practice, $18,213-221$.

Jordan, L., Miller, D., \& Mercer, C. D. (1999). The effects of concrete to semiconcrete to abstract instruction in the acquisition and retention of fraction concepts and skills. Learning Disabilities: A Multidisciplinary Journal, 9(3), 115-122.

Judge, S., \& Watson, S. M. R. (2011). Longitudinal outcomes for mathematics achievement for students with learning disabilities. Journal of Educational Research, 104, 147-157. doi:10.1080/00220671003636729

Kail, R. V. (2004). Cognitive development includes global and domain-specific processes. Merrill-Palmer Quarterly, 50, 445-455. 
Kaiser, G., \& Willander, T. (2005). Development of mathematical literacy: results of an empirical study. Teaching Mathematics and its Applications, 24, 48-60.

Keehn, B., Lincoln, A. J., Muller, R., Townsend, J. (2010). Attentional networks in children and adolescents with autism spectrum disorder. The Journal of Child Psychology and Psychiatry, 51, 1251-1259. doi:10.1111/j.1469.7610.2010.02257.x

Kelly, B., Carnine, D., Gersten, R, S., \& Grossen, B. (1986). The effectiveness of videodisc instruction in teaching fractions to learning disabled and remedial high school students. Journal of Special Education Technology, 8, 5-17.

Kerslake, D. (1986). Fractions: Children's strategies and errors. A report of the strategies and errors in secondary mathematics project. Windsor, England: NFER-Nelson.

Kids Count Data Center (2013). Virginia indicators [Interactive database]. Retrieved from http://datacenter.kidscount.org/data\#VA/2/0

Kieren, T.E. (1976). On the mathematical, cognitive, and instructional foundations of rational numbers. In R. Lesh (Ed.), Number and Measurement: Papers from a Research Workshop (pp. 101-144). Columbus, OH.

Kilpatrick, I, Swafford, J., \& Findell, B. (2001). Adding it up: Helping children learn mathematics. Washington, DC: National Research Council.

Kline, R. B. (2011). Principles and practice of Structural Equation Modeling (23rd ed.). New York: Guilford Press.

Kolikant, Y. B., \& Broza, O. (2011). The effect of using a video clip presenting a contextual story on low-achieving students' mathematical discourse. Educational Studies in Mathematics, 76, 23-47. doi:10.1007/s10649-010-9262-5

Kyttala, M., \& Lehto, J. E. (2008). Some factors underlying mathematical performance: The role 
of visuospatial working memory and non-verbal intelligence. European Journal of Psychology of Education, 23, 77-94.

Lamon, S. J. (2012). Teaching Fractions and Ratios for Understanding, Routledge, New York.

Mabbott, D. J., \& Bisanz, J. (2008). Computational skills, working memory, and conceptual knowledge in older children with mathematics learning disabilities. Journal of Learning Disabilities, 41, 15-28. doi:10.1177/002221940731103

Mazzocco, M. M., \& Devlin, K. T. (2008). Special section: The development of mathematical cognition parts and 'holes': Gaps in rational number sense among children with vs. without mathematical learning disabilities. Developmental Science, 11, 681-691.

Mazzocco, M. M., Feigenson, L., \& Halberda, J. (2011). Impaired acuity of the approximate number system underlies mathematical learning disability (dyscalculia). Child Development, 82, 1224-1237.

McCloskey, M. (2007). Quantitative literacy and developmental dyscalculias. In D. B. Berch \& M. M. M. Mazzocco (Eds.), Why is math so hard for some children? The nature and origins of children's mathematical learning difficulties and disabilities (pp. 415-429). Baltimore, MD: Brookes.

McKinney, J. D., \& Speece, D. L. (1986). Academic consequences and longitudinal stability of behavioral subtypes of learning disabled children. Journal of Educational Psychology, 78, 369-372.

Meyer, M. L., Salimpoor, V. N., Wu, S. S., Geary, D. C., \& Menon, V. (2010). Differential contribution of specific working memory components to mathematics achievement in 2nd and 3rd graders. Learning and Individual Differences, 20, 101-109. doi:10.1016/j.lindif.2009.08.004 
Mezzacappa, E. (2004). Alerting, orienting, and executive attention: Developmental properties and sociodemographic correlates in an epidemiological sample of young, urban children. Child Development, 75, 1373-1386.

Miller, S. C., \& Cook, N. L. (1989). Mainstreaming students with learning disabilities for videodisc math instruction. Teaching Exceptional Children, 21, 57-60.

Morgan, P. L., Farkas, G., \& Wu, Q. (2011). Kindergarten children's growth trajectories in reading and mathematics: Who falls increasingly behind? Journal of Learning Disabilities, 44, 472-480. doi:10.1177/002219411414010

Moore, L. J., \& Carnine, D. (1989). Evaluating curriculum design in the context of active teaching. Remedial and Special Education, 4, 28-37.

Mundia, L. (2010). Learning mathematics: Comparison of Brunei Junior High School students in classes with and without repeaters. Journal of Mathematics Research, 2, 150-160. Retrieved from www.ccsenet.org/jmr

Murnane, R. J., Willett, J. B., \& Levy, F. (1995). The growing importance of cognitive skills in wage determination. Review of Economics and Statistics, 78, 251-266.

Murphy, M. M., \& Mazzocco, M. M. (2008). Rote numeric skills may mast underlying mathematical disabilities in girls with fragile x syndrome. Developmental Neuropsychology, 33, 345-364. doi:10.1080/87565640801982429

Murphy, M. M., Mazzocco, M. M. M., Hanich, L. B., \& Early, M. C. (2007). Cognitive characteristics of children with mathematics learning disability (MLD) vary as a function of the cutoff criterion used to define MLD. Journal of Learning Disabilities, 40, 458478.

National Center for Education Statistics. (2011). The Nations Report Card. Retrieved May, 19 
2013, from http://nces.ed.gov/nationsreportcard/pdf/main2011

National Center for Education Statistics. (2013). The Nations Report Card. Retrieved November 22, 2013, from http://nces.ed.gov/nationsreportcard/itmrlsx/landing.aspx

Ni, Y., \& Zhou, Y. D. (2005). Teaching and learning fraction and ratio numbers: The origins and implications of whole number bias. Educational Psychologist, 40, 27-52.

Norman, G. R., \& Streiner, D. L. (2003). PDQ statistics (3rd ed.). London: BC Decker Inc.

Pantziara, M., \& Philippou, G. (2012). Levels of students" “conception” of fractions. Educational Studies in Mathematics, 79, 61-83. doi: 10.1007/s10649-011-9338-x

Parsons, S., \& Bynner, J. (1997). Numeracy and employment. Education \& Training, 39, 43-51. doi:10.1108/00400919710164125

Passolunghi, M. C., Cornoldi, C., \& De Liberto, S. (1999). Working memory and intrusions of irrelevant information in a group of specific poor problem solvers. Memory and Cognition, 27, 779-790.

Passolunghi, M. C., \& Siegel, L. S. (2004). Working memory and access to numerical information in children with disability in mathematics. Journal of Experimental Child Psychology, 88, 348-367. doi:10.1016/j.jecp.2004.04.002

Passolunghi, M. C., Vercelloni, B., \& Schadee, H. (2007). The precursors of mathematics learning: Working memory, phonological ability and numerical competence. Cognitive Development, 22, 165-184. doi:10.1016/j.cogdev.2006.09.001

Posner, M. I., \& Fan, J. (2008). Attention as an organ system. In J. R. Pomerantz (Ed.), Topics in Integrative Neuroscience: From Cells to Cognition (pp. 31-61).

Posner, M. I., \& Petersen, S. E. (1990). The attention system of the human brain. Annual Review of Neuroscience, 13, 25-42. 
Powell, S. R., Fuchs, L. S., Fuchs, D., Cirino, P. T., \& Fletcher, J. M. (2009). Effects of fact retrieval tutoring on third-grade students with math difficulties with and without reading difficulties. Learning Disabilities Research and Practice, 24, 1-11. doi:10.1111/j.1540$\underline{5826.2008 .01272 . x}$

Rasmussen, C., \& Bisanz, J. (2005). Representation and working memory in early arithmetic. Journal of Experimental Child Psychology, 91, 137-157. doi:10.1016/j.jecp.2005.01.004

Riddle, M., \& Rodzwell, B. (2000). Fractions: What happens between kindergarten and the army? Teaching Children Mathematics. 7, 202-208.

Rittle-Johnson, B., Alibali, M. W. (1999). Conceptual and procedural knowledge of mathematics: Does one lead to the other? Journal of Educational Psychology, 91, 175189.

Rittle-Johnson, B., Siegler, R. S., \& Alibali, M. W. (2001). Developing conceptual understanding and procedural skill in mathematics: An iterative process. Journal of Educational Psychology, 93, 346-362.

Rivera-Batiz, F. L. (1992). Quantitative literacy and the likelihood of employment among young adults in the United States. Journal of Human Resources, 27, 313-328.

Robertson, L., \& Delis, D. C., (1986). Part-whole processing in unilateral brain damaged patients: dysfunction of hierarchical organization. Neuropsychology, 24, 363-370.

Rothbart, M. K., Ellis, L. K., Rueda, M. R., \& Posner, M. I. (2003). Developing mechanisms of effortful control. Journal of Personality, 71, 1113-1143.

Rueda, M. R., Fan, J., Halparin, J., Gruber, D., Lercari, L. P., McCandliss, B. D., et al. (2004). Development of attention during childhood. Neuropsychologia, 42, 1029 - 1040.

Seethaler, P. M., Fuchs, L. S., Star, J. R., \& Bryant, J. (2011). The cognitive predictors of 
computational skill with whole versus rational numbers: An exploratory study. Learning and Individual Differences, 21, 536-542. doi:10.1016/j.lindif.2011.05.002

Siegler R. S., Duncan, G. J., Davis-Kean, P. E., Duckworth, K., Classens, A., Engel, M., ... Chen, M. (2012). Early predictors of high school mathematics achievement. Psychological Science, 23, 691-698. doi:10.1177/0956797612440101

Siegler, R. S., Thompson, C. A., \& Schneider, M. (2011). An integrated theory of whole number and fractions development. Cognitive Psychology, in press.

Smith, J. B. (1996). Does an extra year make any difference? The impact of early access to algebra on long-term gains in mathematics attainment. Educational Evaluation and Policy Analysis, 18, 141-153. doi:10.3102/01623737018002141

Streefland, L.: 1991, Fractions in Realistic Mathematics Education: A Paradigm of Developmental Research (R. Rainero et al., Trans.), Kluwer Academic Publishers, The Netherlands.

Swanson, H. L. (2006) Cross-sectional and incremental changes in working memory and mathematical problem-solving. Journal of Educational Psychology,98, 265-281.

Swanson H. L., \& Beebe-Frankenburger M. (2004). The relationship between working memory and mathematical problem-solving in children at risk and not at risk for serious math difficulties. Journal of Educational Psychology, 96, 471-491.

Swanson, H. L., Jerman, O., \& Zheng, X. (2009). Math disabilities and reading disabilities: Can they be separated? Journal of Psychoeducational Assessment,27, 175-196.

Swanson, H. L., \& Sachse-Lee, C. (2001). Mathematical problem solving and working memory in children with learning disabilities: Both executive and phonological processes are important. Journal of Experimental Child Psychology, 79, 294-321. 
doi:10.1006/jecp.2000.2587

Sweller, J. (1988). Cognitive load during problem solving: Effects on learning. Cognitive Science, 12, 257-285.

Sweller, J., \& Cooper, G. (1985). The use of worked examples as a substitute for problem solving in learning algebra. Cognition and Instruction, 2, 59-89.

Tarver, S. G., Hallahan, D. P., Kauffman, J. M., \& Ball, D. W. (1976). Verbal rehearsal and selective attention in children with learning disabilities: A developmental lag. Journal of Experimental Child Psychology, 22, 375-385.

Test, D. W., \& Ellis, M. F. (2005). The effects of LAP fractions on addition and subtraction of fractions with students with mild disabilities. Education \& Treatment of Children, 28, $11-24$.

Tirosh, D. (2000). Enhancing prospective teachers' knowledge of children's conceptions: The case of division of fractions. Journal for Research in Mathematics Educaiton, 31, 5-25.

Tolar, T. D., Fuchs, L., Cirino, P. T., Fuchs, D., Hamlett, C. L., \& Fletcher, J. M. (2012). Predicting development of mathematical word problem solving across the intermediate grades. Journal of Educational Psychology, 104, 1083-1093. doi:10.1037/a0029020

Toll, S. W. M., Van der Ven, S. H. G., Kroesbergen, E. H., \& Van Luit, J. E. H. (2011). Executive functions as predictors of math learning disabilities. Journal of Learning Disabilities, 44, 521-530. doi:10.1177/0022219410387302

Tronsky, L. N. (2005). Strategy use, the development of automaticity, and working memory involvement in complex multiplication. Memory \& Cognition, 33, 927-940.

U.S. Census Bureau (2014). State \& County Quick Facts. Retrieved from http://quickfacts.census.gov/qfd/states/.html 
U.S. Department of Education, National Center for Education Statistics (2012). Digest of Education Statistics, 2011 (NCES 2012-001), Chapter 2.

Virginia Department of Education (2013). Fall membership for the Commonwealth of Virginia. [Interactive database]. Retrieved from http://bi.vita.virginia.gov/doe bi/rdPage.aspx?rdReport=Main\&subRptName=Fallmembe $\underline{\text { rship }}$

Virginia Department of Education (2014). Mathematics Standards of Learning. Retrieved from http://www.doe.virginia.gov/testing/sol/standards_docs/mathematics/

Vukovic, R. K., \& Siegel, L. S. (2010). Academic and cognitive characteristics of persistent mathematics difficulty from first through fourth grade. Learning Disabilities Research \& Practice, 25, 25-38. doi:10.11111j.1540-5826.2009.00298.x

Wagner, M., Newman, L., Cameto, R., Levine, P., \& Garza, N. (2006). An Overview of Findings From Wave 2 of the National Longitudinal Transition Study-2 (NLTS2). Menlo Park, CA: SRI International. Available at Www.nlts2.org/reports/2006_08/nlts2_report_2006_08_complete.pdf

Watson, S. M. R., \& Gable, R. A. (2012). Unraveling the complex nature of mathematics learning disability: Implications for research and practice. Learning Disability Quarterly, 36, 178-187. doi:10.1177/07311948712461489

Wechsler, D. (1999). Wechsler Abbreviated Scale of Intelligence. San Antonio, TX: Psychological Corporation.

Wentzel, K. R. (1991). Relations between social competence and academic achievement in early adolescence. Child Development, 62, 1066-1078.

Wilkinson, G. S., \& Robertson, G. J. (2006). Wide Range Achievement Test 4 professional 
manual. Lutz, FL: Psychological Assessment Resources.

Wolf, M., \& Bowers, P. G. (1999). The double-deficit hypothesis for the developmental dyslexias. Journal of Educational Psychology, 91, 415-438.

Woodcock, R. W., McGrew, K. S., Mather, N. (2001) Woodcock-Johnson III: Complete Battery Tests of Achievement. Itasca, IL: Riverside Publishing.

Woodward, J., Baxter, J., \& Robinson, R. (1999). Rules and reasons: Decimal instruction for academically low achieving students. Learning Disabilities Research \& Practice, 14, 15 24.

Woodward, J. \& Gersten, R. (1992). Innovative technology for secondary learning disabled students: A multi-faceted study of implementation. Exceptional Children, 58, 407-421.

Wu, H. (2008). Fractions, decimals, and rational numbers. Retrieved May 5, 2013, from University of California, Berkeley, Department of Mathematics Web site: http://math.berkeley.edu/ wu/

Xin, Y. R, Jitendra, A. K., \& Deatline-Buchman. A. (2005). Effects of mathematical word problem-solving instruction on middle school students with learning problems. The Journal of Special Education, 39,181-192.

Zentall, S. S. (1993). Research on the educational implications of attention deficit hyperactivity disorder. Exceptional Children, 60, 143-153.

Zorzi, M., Priftis, K., \& Umilta, C. (2002). Neglect disrupts the mental number line. Nature, 417, $138-140$. 


\section{APPENDIX}

Figure 9

Path Analysis Model with Standardized Regression Weights and Covariances

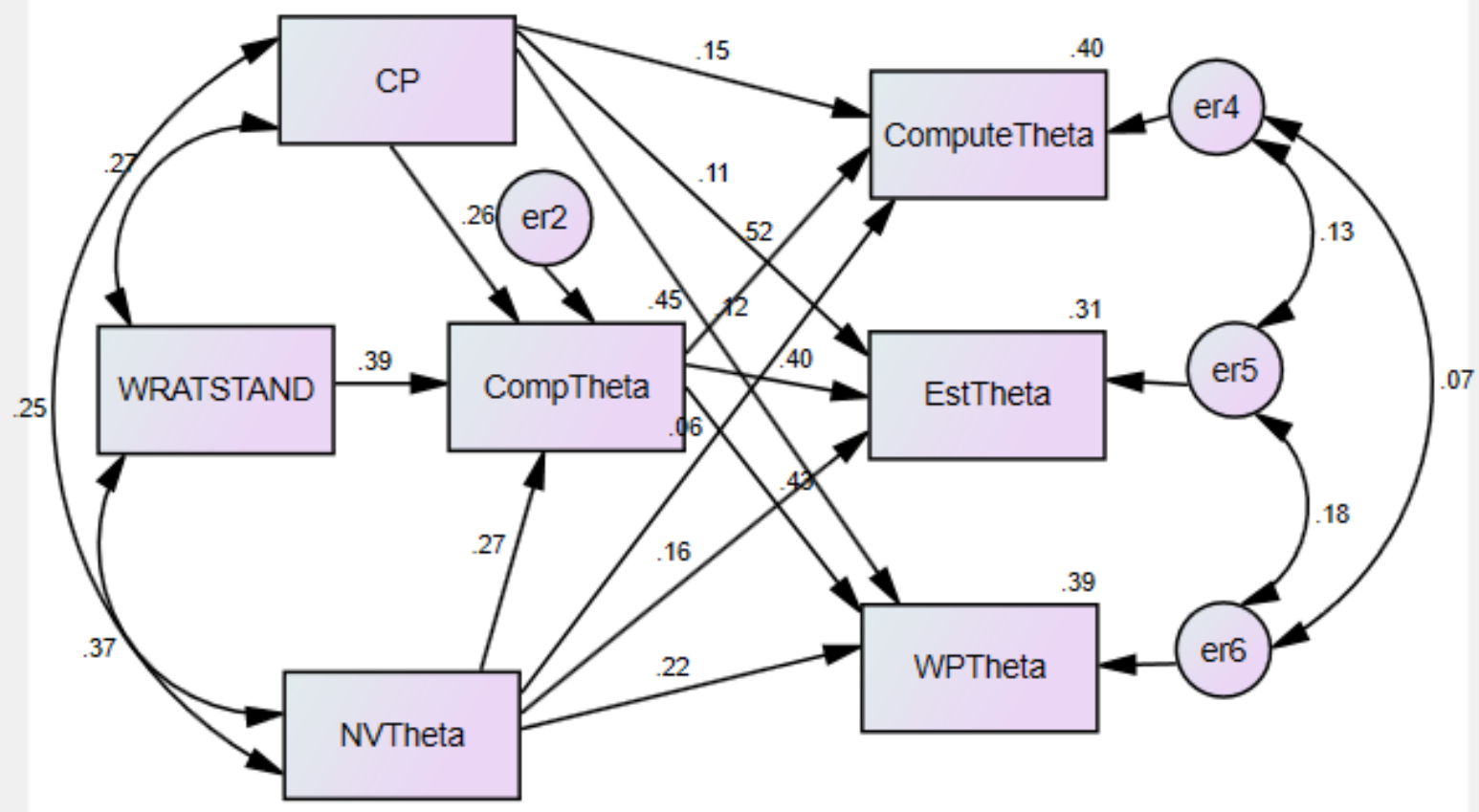


Table 4

Means, Standard Deviations, Inter-Item Covariance Matrix and Simple Correlations Among Variables

\begin{tabular}{lcccccc}
\hline Variable & M (S.D.) & 1 & 2 & 3 & 4 & 5 \\
\hline & & & & & & \\
1. WRAT-4 standard. & $110.84(12.97)$ & 155.852 &. $\mathbf{2 6 8}$ & $\mathbf{. 3 6 7}$ & $\mathbf{. 5 5 5}$ & $\mathbf{. 3 7 8}$ \\
2. d2 Test of Attention & $130.4(26.67)$ & 88.258 & 694.574 & $\mathbf{. 2 5 4}$ & $\mathbf{. 4 2 8}$ & $\mathbf{. 3 9 2}$ \\
3. Nonverbal reasoning & $1.035(1.11)$ & 5.066 & 7.398 & 1.224 & $\mathbf{. 4 7 3}$ & $\mathbf{. 3 4 3}$ \\
4. Conceptual & $0.888(1.96)$ & 13.537 & 22.021 & 1.023 & 3.815 & $\mathbf{. 6 1 6}$ \\
5. Calculation & $0.622(2.23)$ & 10.512 & 23.032 & .846 & 2.681 & 4.964 \\
6. Estimation & $0.341(2.44)$ & 9.286 & 20.61 & 1.018 & 2.499 & 2.345 \\
7. WP set-up & $2.081(2.17)$ & 12.35 & 20.497 & 1.079 & 2.473 & 2.077
\end{tabular}

Note: Items bolded and italicized are correlations and items not bolded or italicized are inter-item covariances 
Table 5

DIF Scores: Examining Items for Bias

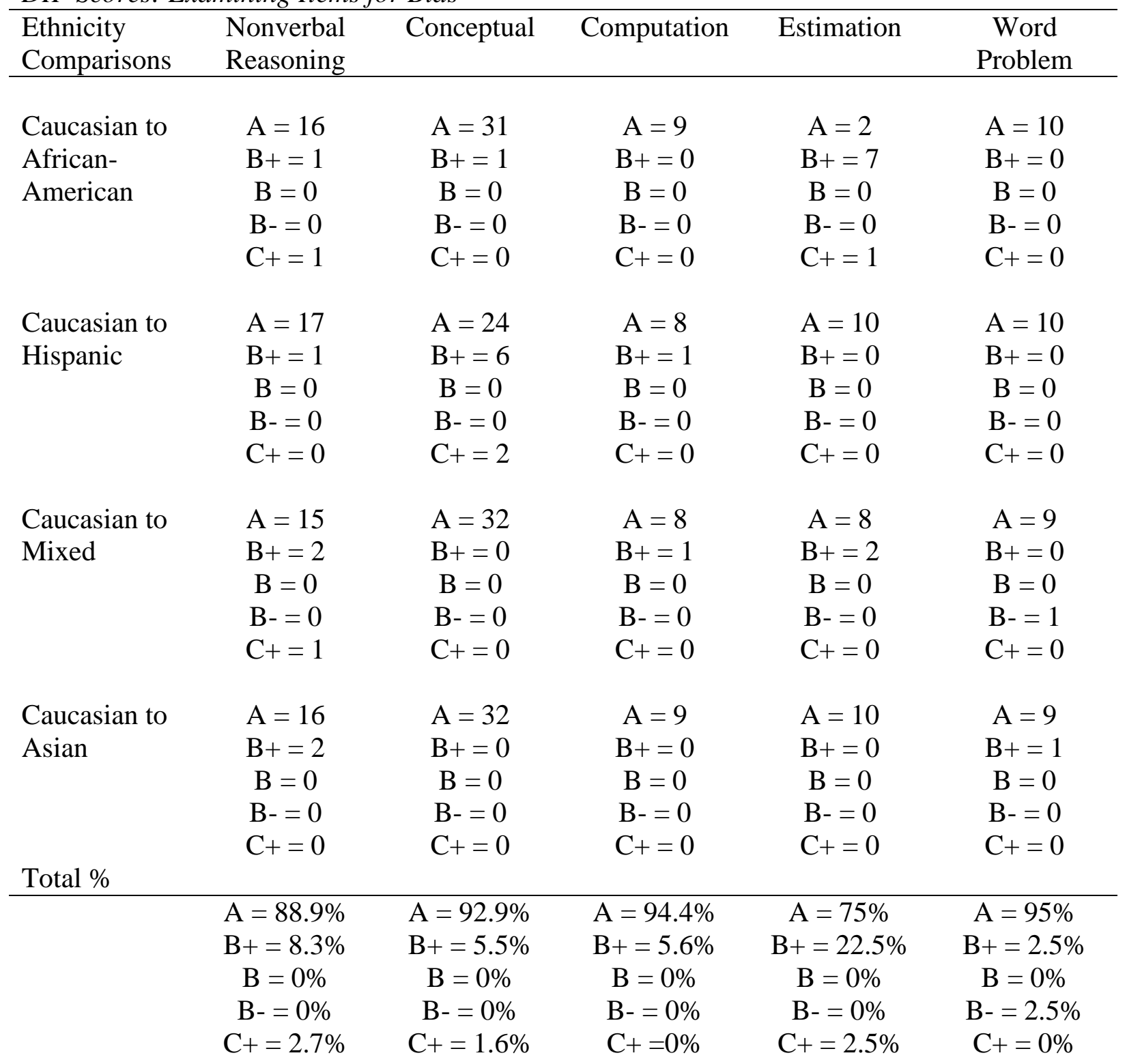




\section{Appendix B}

Study Assessment Materials

Name: 
Participant \# 


\section{Fraction Conceptual Understanding Assessment}

Draw a picture that represents the fraction written in each box. Use circles or rectangles to represent each fraction.
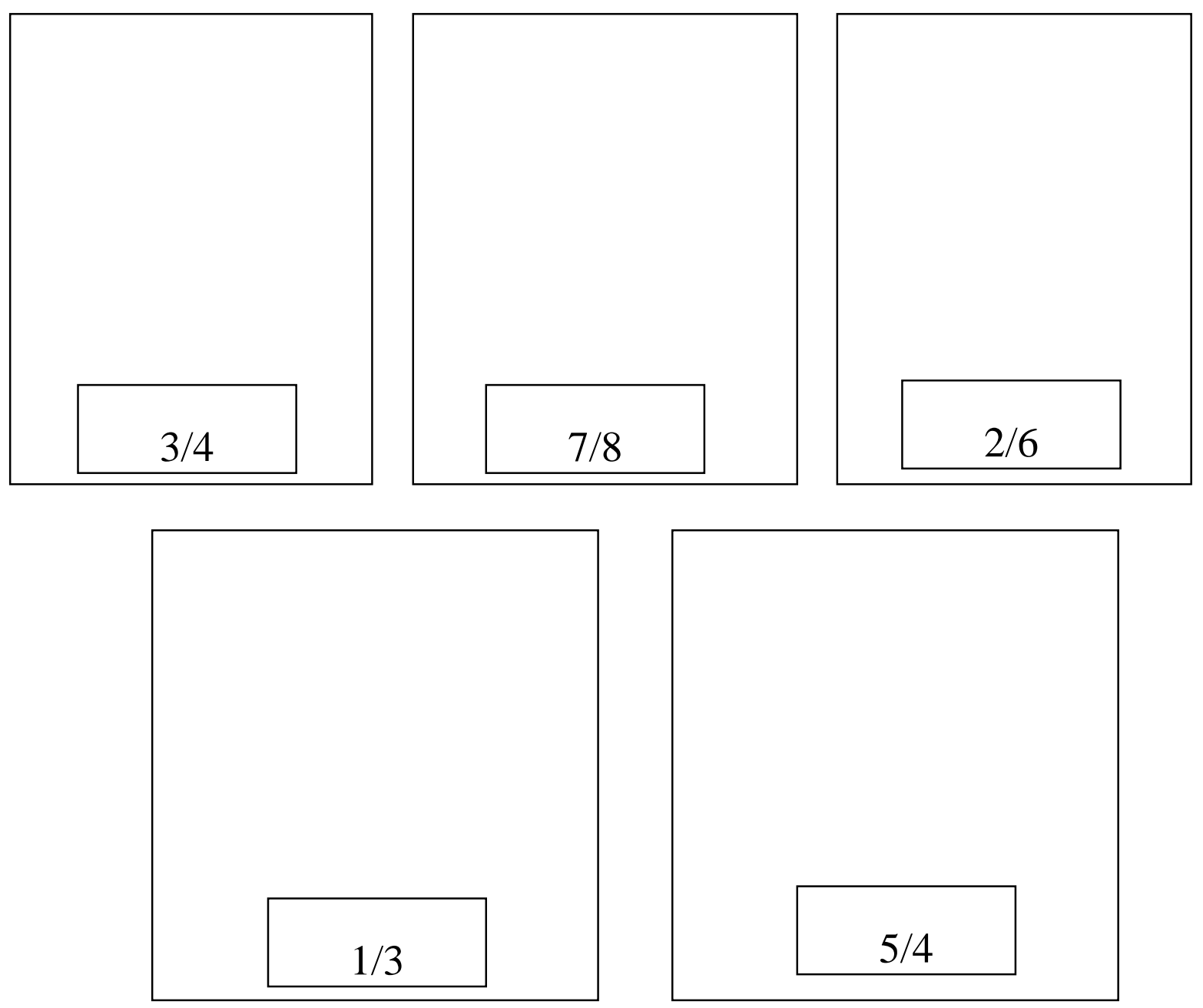
Write a fraction representing the shaded portion's part of the whole
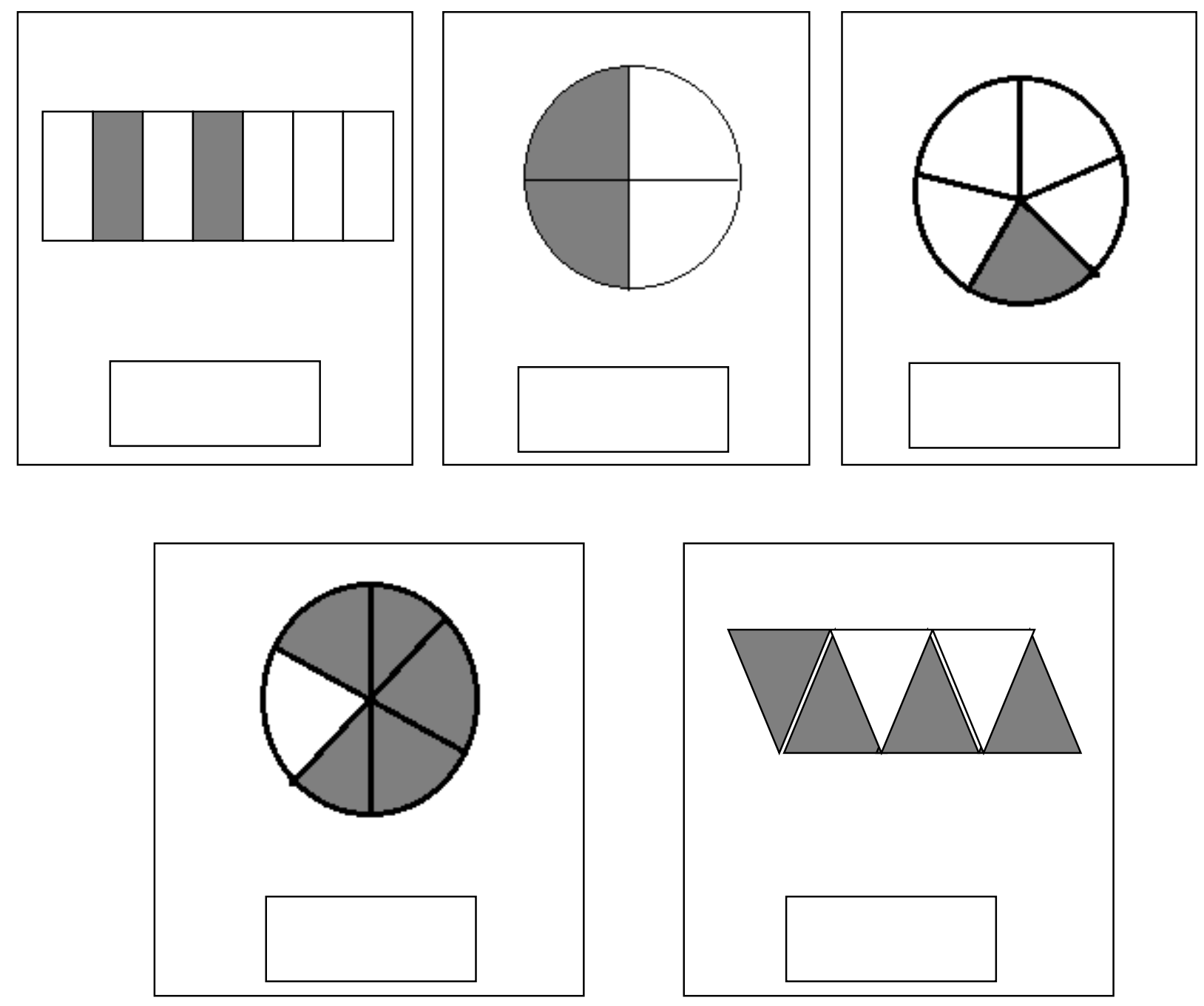

Mark the appropriate spot on the number line for each given fraction

11) $\frac{3}{8}$

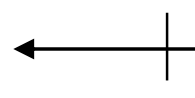


12) $\frac{4}{5}$

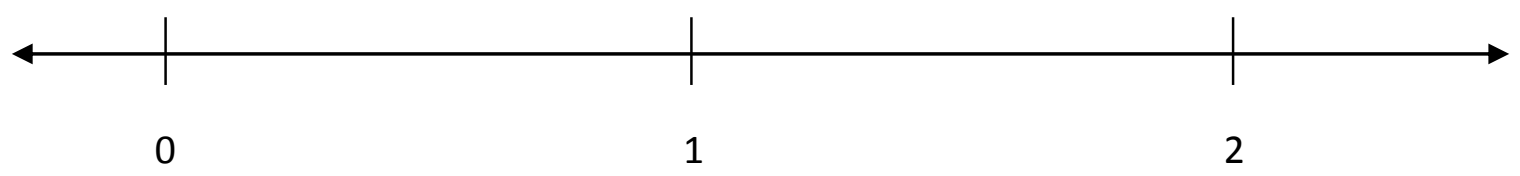

13) $\frac{6}{7}$

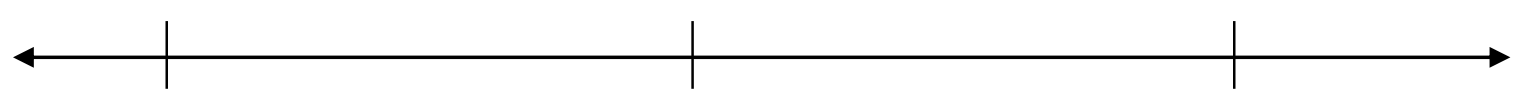

0

1

14) $\frac{6}{3}$

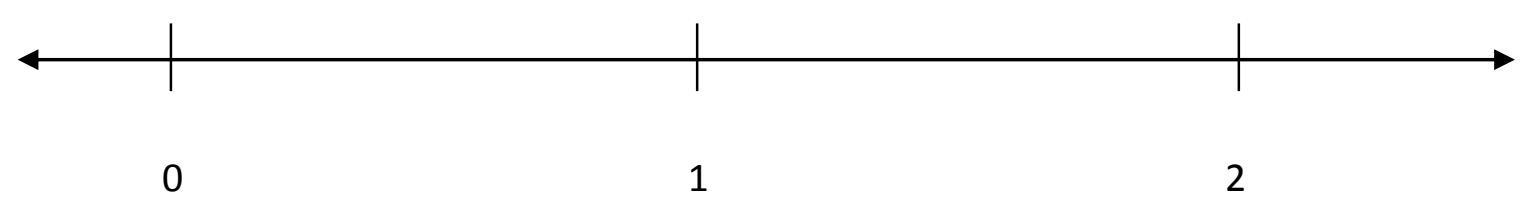

Circle the larger fraction. If the fractions are equal to each other circle both fractions

15) $\frac{1}{2} \quad \frac{1}{3}$

16) $\frac{3}{5} \quad \frac{4}{5}$ 
17) $2 \frac{2}{6} \quad 2 \frac{1}{3}$

18) $\frac{6}{7} \quad \frac{7}{6}$

19) Which is equivalent to $\frac{4}{5}=B_{\text {? }}$
A. $\frac{16}{20}$
B. $\frac{4}{8}$
C. $\frac{8}{12}$
D. $\frac{8}{15}$

20) Which is equivalent to $\frac{6}{2}=$
A. $\frac{14}{7}$
B. $\frac{6}{8}$
C. 11
D. 3

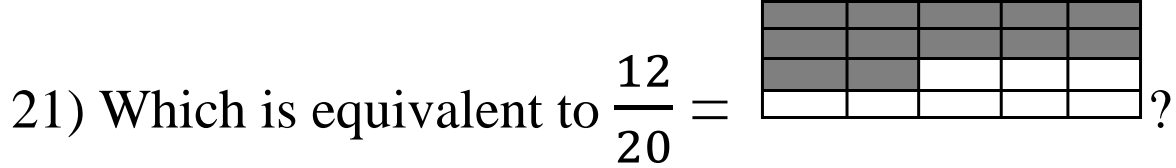
A. $\frac{6}{8}$
B. $\frac{3}{5}$
C. $\frac{4}{12}$
D. $\frac{8}{15}$ 
22) Which is equivalent to $2 \frac{2}{6}=$
A. $\frac{16}{8}$
B. $\frac{3}{8}$
C. $\frac{7}{3}$
D. $\frac{5}{2}$

Solve these addition and subtraction problems by drawing lines in the last figure and shading the appropriate areas.

23) Find the sum of the first two figures.

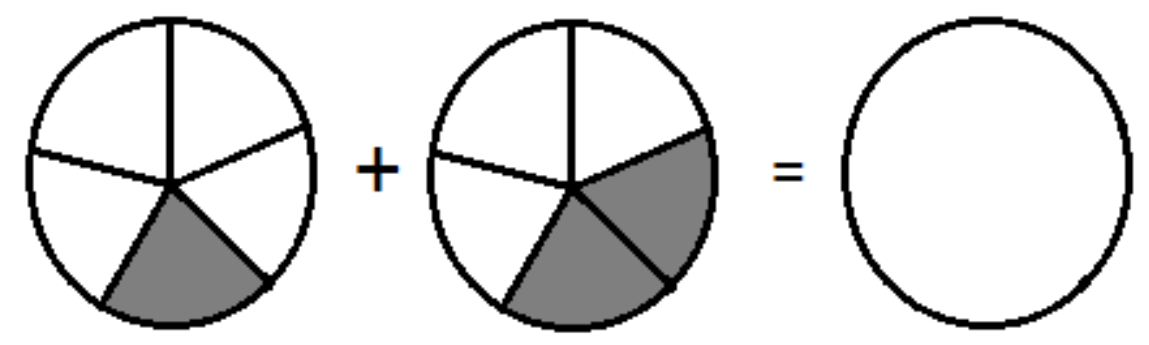

24) Find the difference between the first two figures

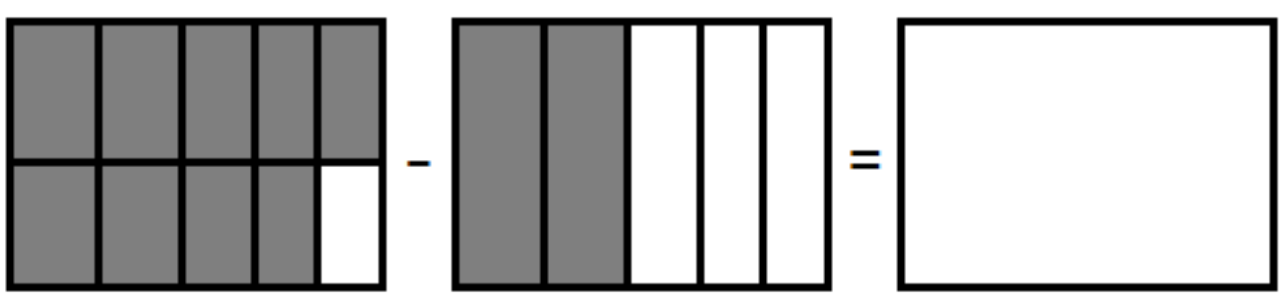


25) Find the difference between the first two figures.
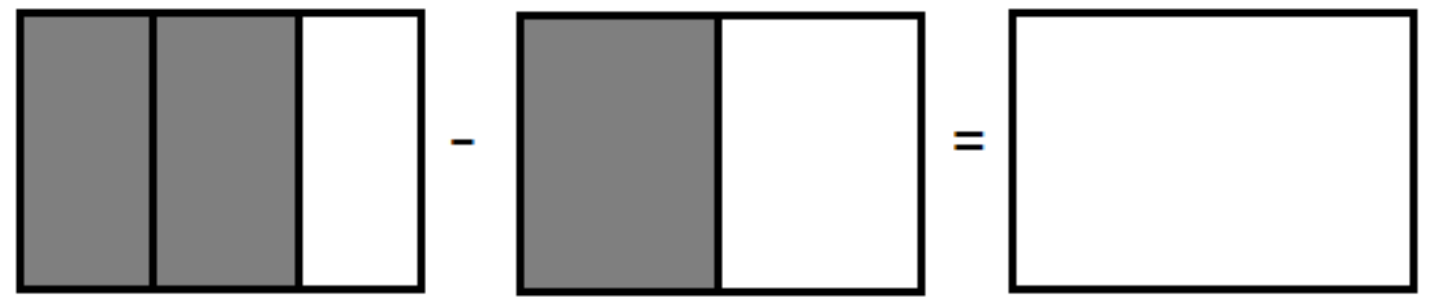

26) Which fraction represents the number of triangles compared to the total number of objects?
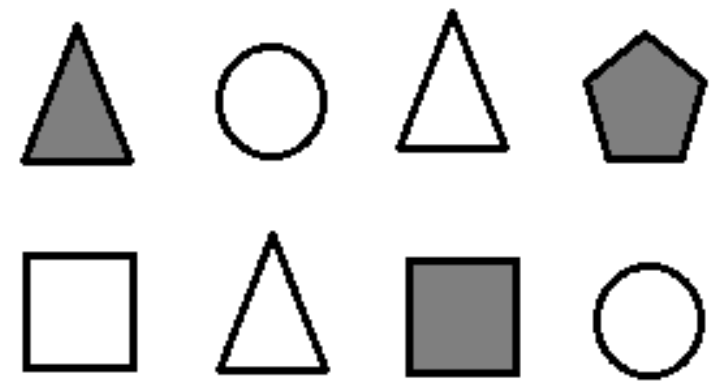
A. $\frac{3}{8}$
B. $\frac{2}{3}$
C. $\frac{3}{5}$
D. $\frac{2}{1}$ 
27) What fraction represents the part of the total group of objects is not shaded?
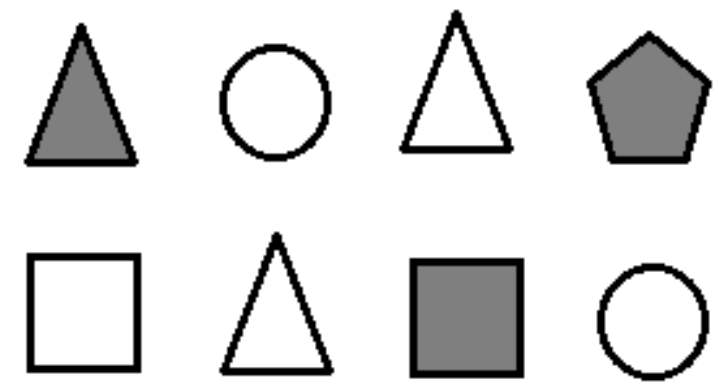
A. $\frac{3}{8}$
B. $\frac{5}{3}$
C. $\frac{5}{8}$
D. $\frac{3}{5}$

28) What part of the group of objects is shaded or circles?
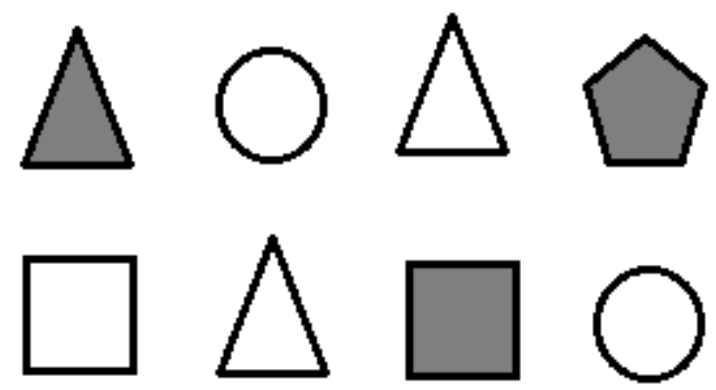
A. $\frac{1}{2}$
B. $\frac{5}{8}$
C. $\frac{2}{16}$
D. $\frac{3}{8}$ 


\section{Nonverbal Reasoning}

In each row there are six figures. Circle the figure that unlike the other figures. You may only circle one answer for each question.

1.

\begin{tabular}{|c|c|c|c|c|c|}
\hline & $\longrightarrow$ & & $\uparrow$ & \\
\hline $\mathrm{A}$ & $\mathrm{B}$ & $\mathrm{C}$ & $\mathrm{D}$ & $\mathrm{E}$ & $\mathrm{F}$ \\
\hline
\end{tabular}

2.

\begin{tabular}{|l|l|l|l|l|l|}
\hline$\square$ & & & $\bigwedge$ & & \\
& & & \\
\hline $\mathrm{A}$ & $\mathrm{B}$ & $\mathrm{C}$ & $\mathrm{D}$ & $\mathrm{E}$ & $\mathrm{F}$ \\
\hline
\end{tabular}

3.

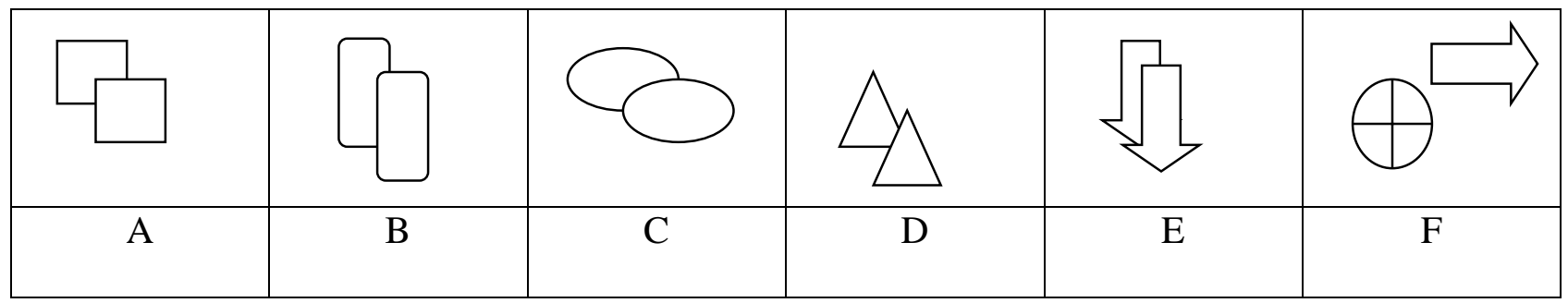


4.

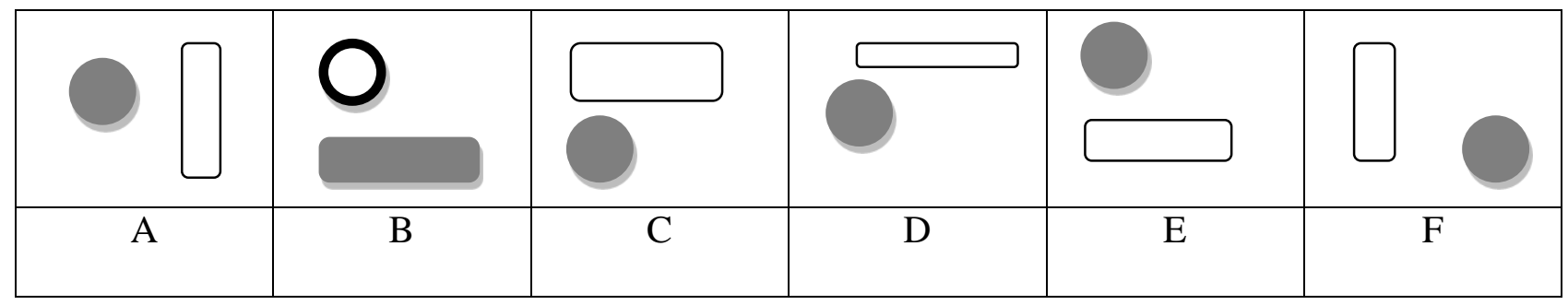

5.

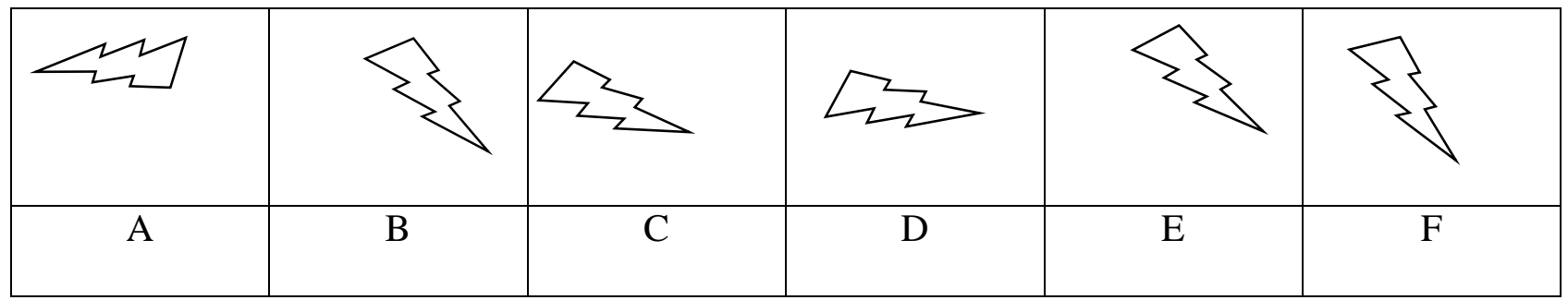

For each of the following questions identify what shape would come next in the pattern

6.

\begin{tabular}{|l|l|l|l|l|l|}
\hline$\square \leadsto 0$ & & & & & \\
\hline
\end{tabular}

7.

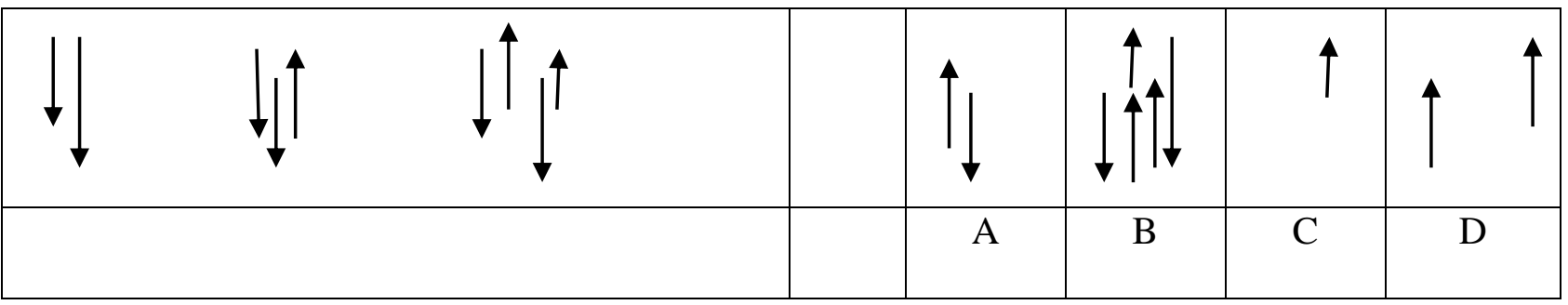


8.

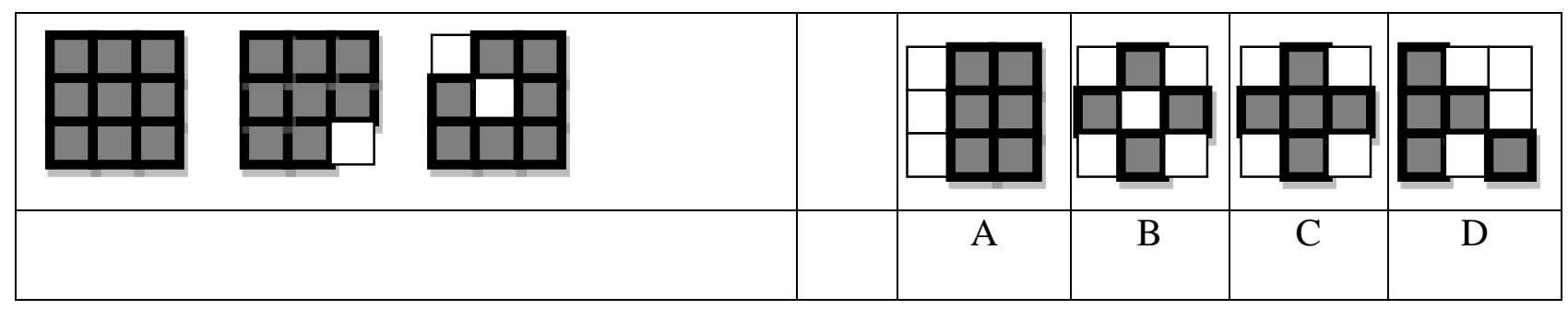

9.

\begin{tabular}{|l|l|l|l|l|l|}
\hline$\langle>0$ & & & & \\
\hline
\end{tabular}

10.

\begin{tabular}{|c|c|c|c|c|c|c|}
\hline & $\mathrm{C}$ & $\mathrm{B}$ & $\mathrm{B}$ \\
\hline
\end{tabular}

For the following problems choose the answer that reflects what the picture would look like if it was turned clockwise 90 degrees (1/4 to the right).

11.

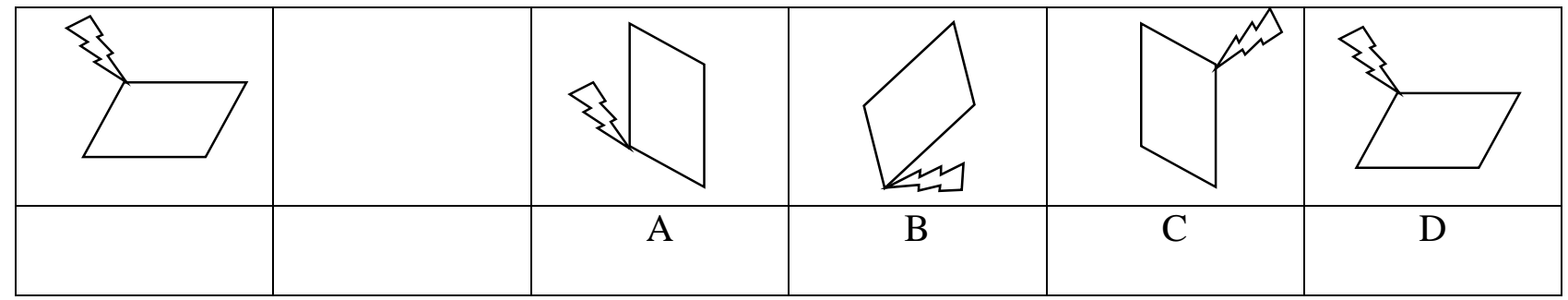


12.

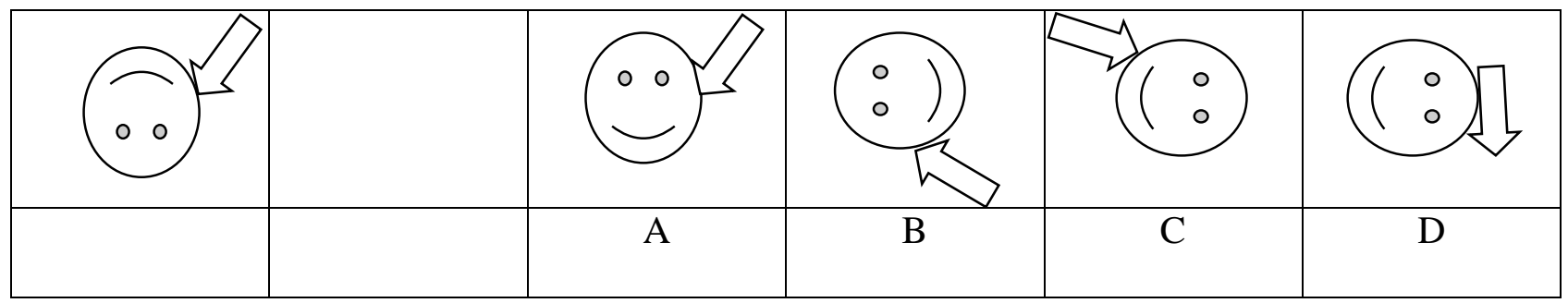

13.

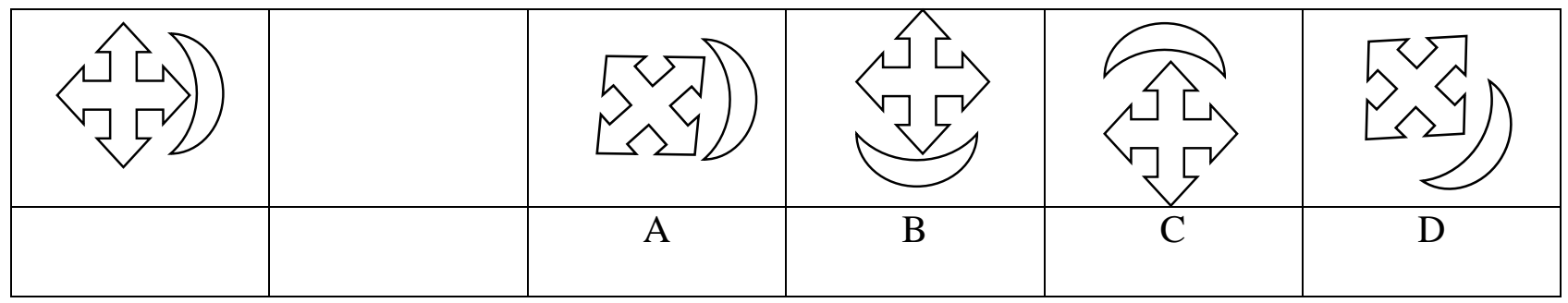

For the following questions choose the shapes that are different from the other shapes.

14.

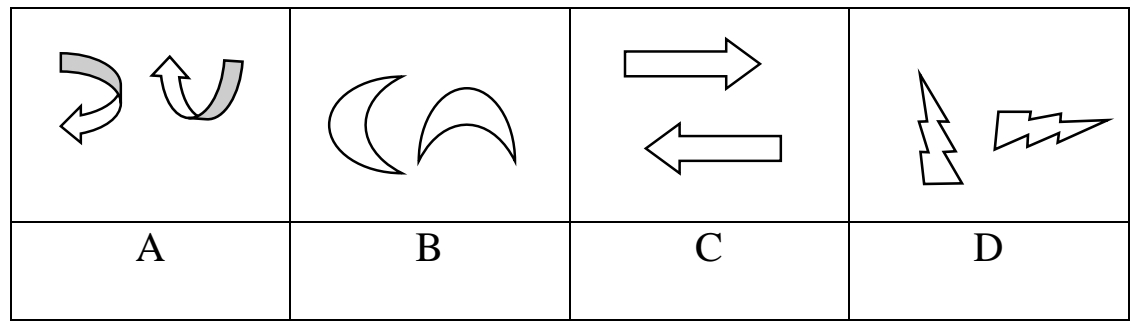

15.

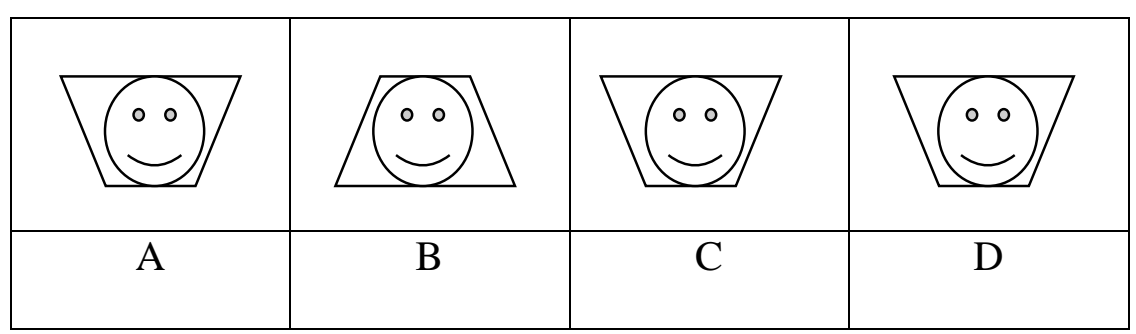


16.

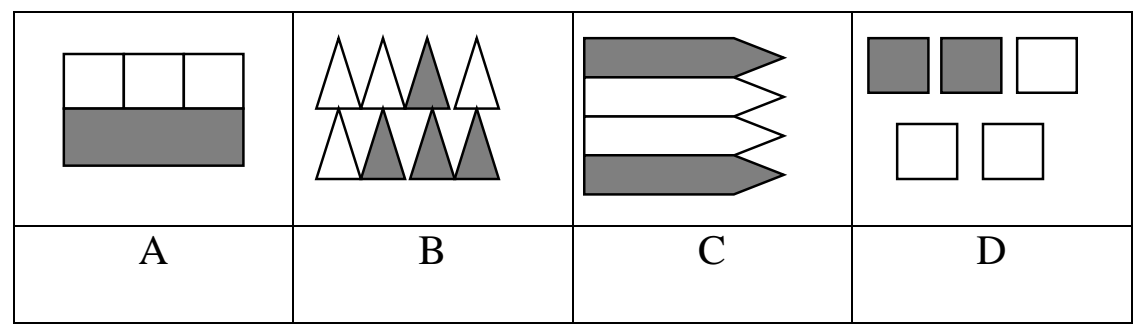

17.

\begin{tabular}{|l|l|l|l|}
\hline & \multicolumn{4}{|}{$\Delta$} & $\Delta$ \\
\hline $\mathrm{A}$ & $\square$ & $\triangle$ \\
\hline
\end{tabular}

Choose the answer that best represents what you would get when putting the two shapes together.

18.

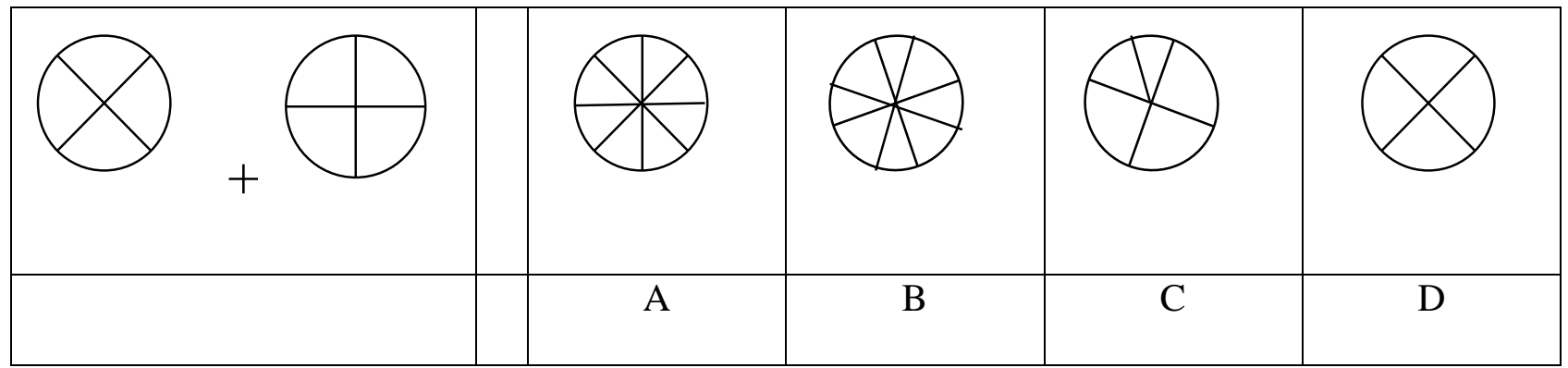




\section{Fraction Word Problems}

Read each word problem and write down the steps you would take to solve each problem. Write the fractions in the appropriate space and choose whether you would solve the problem by addition, subtraction, multiplication, or division. For example:

Randy had 3/4 of a bag of his favorite chips and his friend gives him another $1 / 2$ of a bag of chips. How many bags of chips does Randy have?

$3 / 4 \quad \frac{+}{+,-, \times, \div}+1 / 2$.

1) Jessica bought $8 / 9$ of a pound of chocolates and ate $1 / 3$ of a pound. How much was left?

$\overline{\text { Fraction }} \overline{+,-, \times, \div} \overline{\text { Fraction }}$

2) Tom bought a board that was $7 / 8$ of a yard long. He cut off $1 / 2$ of a yard. How much was left?

$\overline{\text { Fraction }} \overline{+,-, \times, \div} \overline{\text { Fraction }}$ 
3) Sam rode his bike $2 / 5$ of a mile and walked another $3 / 4$ of a mile. How far did he travel?

$\overline{\text { Fraction }} \overline{+,-, x, \div} \overline{\text { Fraction }}$

4) Sally walked $3 / 4$ of a mile before lunch and $1 / 2$ of a mile after lunch. How far did she walk in all?

$\overline{\text { Fraction }} \overline{+,-, \times, \div} \overline{\text { Fraction }}$

5) Don bought 3/4 of a pound of jellybeans and $5 / 8$ pound of gummy bears. How much candy did he buy?

$\overline{\text { Fraction }} \overline{+,-, \times, \div} \overline{\text { Fraction }}$

6) The track is $3 / 5$ of a mile long. If Tyrone jogged around it twice, how far did he run?

$\overline{\text { Fraction }} \overline{+,-, \times, \div} \overline{\text { Fraction }}$

7) Stanley ordered two pizzas cut into eighths. If he ate $5 / 8$ of a pizza, how much was left?

$\overline{\text { Fraction }} \overline{+,-, \times, \div} \overline{\text { Fraction }}$ 
8) Sandra bought $23 / 4$ yards of red fabric and $11 / 4$ of blue. How much cloth did she buy in all?

$\overline{\text { Fraction }} \overline{+,-, \times, \div} \overline{\text { Fraction }}$

9) An equilateral triangle measures $31 / 2$ inches on one side. What is the perimeter of the triangle?

$\overline{\text { Fraction }} \overline{+,-, \times, \div} \overline{\text { Fraction }}$

10) You have $11 / 3$ pizzas left after a party. You only have $1 / 3$ of the amount of pizza you started with left? How much pizza did you start with?

$\overline{\text { Fraction }} \overline{+,-, \times, \div} \overline{\text { Fraction }}$ 
Fraction

Computation

1)

$\frac{5}{8}+\frac{2}{8}$

4)

$\frac{3}{4}+\frac{2}{8}$

7)

$\frac{1}{2} \times \frac{4}{5}$
2)

$\frac{3}{6}+\frac{1}{6}$

5)

$2 \frac{1}{3}+1 \frac{1}{3}$

8)

$\frac{4}{7} \times \frac{3}{2}$
3)

$\frac{1}{2}+\frac{1}{3}$

6)

$\frac{5}{6} \times \frac{2}{4}$

9)

$\frac{2}{5} \times \frac{1}{2}$ 
Name

Date

Estimating Sums and Differences with Fractions - Matching

Worksheet

Write the letter of the answer that matches the problem.

1. Estimate the sum of the fractions.
$9 \frac{2}{4}+4 \frac{3}{9}$
a. 1

2. Estimate the difference of the fractions.

$$
6 \frac{4}{5}-1 \frac{1}{2}
$$

b. 32

3. Estimate the difference of the

fractions $\frac{6}{7}-\frac{6}{18}$

c. 37

4. Estimate the sum of the fractions.

$\frac{4}{16}+\frac{22}{44}$

d. 6

5. Estimate the difference of the fractions.

$$
12 \frac{3}{4}-6 \frac{2}{4}
$$

e. 5

6. Estimate the sum of the fractions.

$$
\frac{2}{3}+\frac{24}{25}
$$

f. $\frac{1}{2}$

7. Estimate the sum of the fractions.

$$
33 \frac{6}{15}+14 \frac{6}{8}
$$

g. 48

8. Estimate the sum of the fractions.

$$
24 \frac{2}{6}+8 \frac{1}{3}
$$

h. 5

9. Estimate the difference of the fractions.

$$
11 \frac{6}{8}-6 \frac{3}{6}
$$

i. 2

10. Estimate the sum of the fractions.

$$
16 \frac{5}{6}+19 \frac{9}{15}
$$

j. 14 


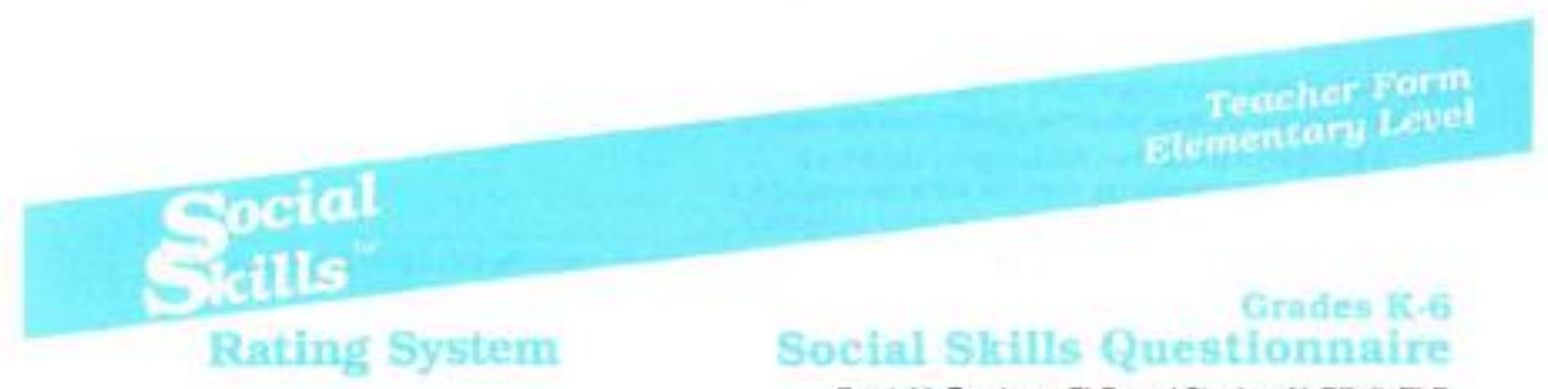

Frank M. Gresham, PhD, and Stephen N. Elion, PhD

\section{Directions}

This questionnaire is designed to measure how often a student oxhibits onrtain social skils and how important those skils are for success in your classroom. Flatings of problem behaviors and boademic competence are also requested. First, complete the information about the student and yourselt.

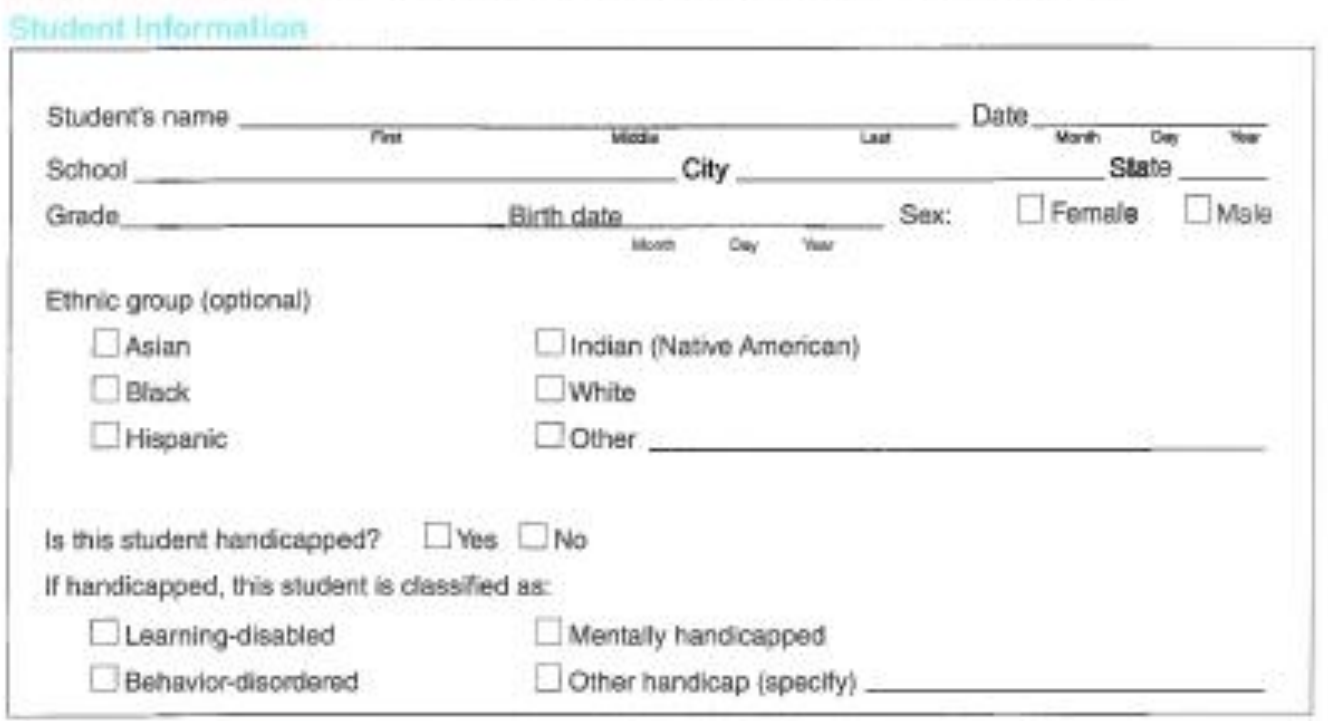

\section{Teactuer inglombition}

Teacher's name

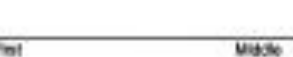
Sex: $\square$ Female $\square$ Mals

What is your assignment?
$\square$ Regular
$\square$ Resaurce
$\square$ Self-contained
$\square$ Other (specily)

\section{PEARSON}

Prychcerp is an imprite of Pearson Clinical Assessment.

Dearsen Executive Office S6b1 Green Valbey Drive Bloomington, MN 55437 800.627.7271 www. Psychcerp.cem

Coppright 01990 NCS Pearson, inc. Al righes restrved.

Warning: No part of bia pubisatian mey be resrodused ar traramithed it any form or by any

mesne, eectren'c or mectianical, induding ghotocogy, recording, or asy information starege and

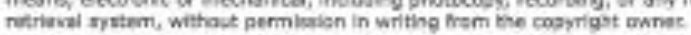

Pearsan, the PSI loga, PeychCorp, and SsRs are trndemarks ir the U.5. andior gther

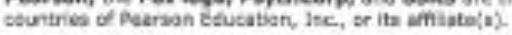

Printad in the Unibes Statan ef A-erica. 
Next, read aach item on peges 2 and 3 (hems 1-48) and think about this shodenfs behavior during the past month or two. Decide how often the student does the behavior described.

If the student never does this behavior, circle the 0 .

If the studant sometimes does this behavior, circle the 1 .

If the student very often does this behavior, circle the 2 .

For items 1-30, you should also rate how important each of these behaviors is for success in your classroom.

If the behavior is not important for sucoess in your classroom, circle the 0 .

If the behavior is important Sor success in your classroom, circle the 1.

If the behavior is critical for success in your ciassroom, circle the 2.

Here are two examples:

\begin{tabular}{|c|c|c|c|c|c|c|}
\hline & \multicolumn{3}{|c|}{$\begin{array}{l}\text { How } \\
\text { Ollen? }\end{array}$} & \multicolumn{3}{|c|}{$\begin{array}{l}\text { How } \\
\text { Impertant? }\end{array}$} \\
\hline & never & Sereutinua & 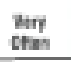 & $\lim _{\text {intort }}$ & linprat & trtal \\
\hline Shows empathy for poers. & 0 & 1 & (2) & 0 & (1) & 2 \\
\hline $\begin{array}{l}\text { Asks questions of you when unsure of what to } \\
\text { do in schoalwork. }\end{array}$ & 0 & & 2 & 0 & 1 & (2) \\
\hline
\end{tabular}

Piease do not skip any items. In some cases you may net have observed the student perform a particular behavior. Make an estimate of the degree to which you think the student would probably perform that behavior.

\begin{tabular}{|c|c|c|c|c|c|c|}
\hline \multicolumn{3}{|c|}{ 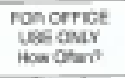 } & \multirow[t]{2}{*}{ Social Skills } & \multirow{2}{*}{\multicolumn{2}{|c|}{$\begin{array}{c}\text { How } \\
\text { Othan? } \\
\text { sarmata }\end{array}$}} & \multirow{2}{*}{$\begin{array}{l}\text { Wery } \\
\text { Ots }\end{array}$} \\
\hline C & A & S & & & & \\
\hline & & & 1. Controls bemper in conflict stustions with poors. & 0 & 1 & 2 \\
\hline & & & $\begin{array}{l}\text { 2. Introduces hergelf or himseit to new poople without } \\
\text { being told. }\end{array}$ & 0 & 1 & 2 \\
\hline & & & 3. Appropriataly questions rules that may be untair. & 0 & 1 & 2 \\
\hline & & & $\begin{array}{l}\text { 4. Compromises in conflict situations by changing } \\
\text { can ideses to reach agreement. }\end{array}$ & $\underline{0}$ & 1 & 2 \\
\hline & & & 5. Ansponds appropriately to peer pressure. & 0 & 1 & 2 \\
\hline & & & $\begin{array}{l}\text { E. Says nice thinge about himsalt or harsalf when } \\
\text { appropriate. }\end{array}$ & 0 & 1 & 2 \\
\hline & & & 7. Imbes othars to join in activities. & 0 & 1 & 2 \\
\hline & & & 8. Uses froe time in an acceptable way. & 0 & 1 & 2 \\
\hline & & & 9. Finishes class assignmants within time limits. & 0 & 1 & 2 \\
\hline & & & 10. Makas friends easily. & 0 & 1 & 2 \\
\hline & & & 11. Aesponds appropriately to leasing by peera. & 0 & 1 & 2 \\
\hline & & & 12. Controls temper in conflict stuations with adults. & 0 & 1 & 2 \\
\hline & & & 13. Aecehes criticism weil. & 0 & 1 & 2 \\
\hline & & & 14. Initiatos corwersations with peers. & 0 & 1 & 2 \\
\hline & & & 15. Uses time apprepriately while waiting for halp. & 0 & 1 & 2 \\
\hline & & & 16. Produces comoct schoalwark. & 0 & 1 & 2 \\
\hline $\mathrm{c}$ & A & 5 & 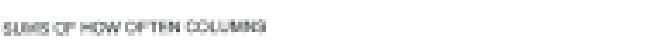 & & & \\
\hline
\end{tabular}

\begin{tabular}{|c|c|c|}
\hline \multicolumn{3}{|c|}{$\begin{array}{c}\text { How } \\
\text { Important? }\end{array}$} \\
\hline$\underset{\text { Irpotax }}{\text { Wx }}$ & Importie & Crtial \\
\hline 0 & 1 & 2 \\
\hline 0 & 1 & 2 \\
\hline 0 & 1 & 2 \\
\hline 0 & 1 & 2 \\
\hline D & 1 & 2 \\
\hline 0 & 1 & 2 \\
\hline 0 & 1 & 2 \\
\hline 0 & 1 & 2 \\
\hline 0 & 1 & 2 \\
\hline 0 & 1 & 2 \\
\hline 0 & 1 & 2 \\
\hline 0 & 1 & 2 \\
\hline 0 & 1 & 2 \\
\hline D & 1 & 2 \\
\hline 0 & 1 & 2 \\
\hline 0 & 1 & 2 \\
\hline
\end{tabular}




\begin{tabular}{|c|c|c|c|c|c|c|c|c|c|}
\hline \multicolumn{3}{|c|}{$\begin{array}{l}\text { Ron once } \\
\text { ust cons } \\
\text { how chen? }\end{array}$} & \multirow[t]{2}{*}{ Social Skills (cont.) } & \multirow{2}{*}{\multicolumn{2}{|c|}{$\begin{array}{l}\text { How } \\
\text { Often? }\end{array}$}} & \multirow[b]{2}{*}{$\underset{\text { aten }}{\text { Men }}$} & \multicolumn{3}{|c|}{$\begin{array}{l}\text { How } \\
\text { Important? }\end{array}$} \\
\hline c & A & S & & & & & $\underset{\text { Inpatant }}{N x}$ & Iaxotant & Ertal \\
\hline & & & $\begin{array}{l}\text { 17. Appropriately tolls you when he of she thirks you } \\
\text { have treased him or her unfaily. }\end{array}$ & 0 & 1 & 2 & 0 & 1 & 2 \\
\hline & & & 16. Accepts poers' ideas for group activifies. & 0 & 1 & 2 & 0 & 1 & 2 \\
\hline & & & 19. Givos complimants to peers. & 0 & 1 & 2 & 0 & 1 & 2 \\
\hline & & & 20. Follows your directione. & $\mathbf{0}$ & 1 & 2 & 0 & 1 & 2 \\
\hline & & & 21. Puts work materials or school property away. & 0 & 1 & 2 & 0 & 1 & 2 \\
\hline & & & 22. Cooperaies with peors without prompting. & 0 & 1 & 2 & 0 & 1 & 2 \\
\hline & & & 23. Volumeers to help peers with classrcom tasks. & 0 & 1 & 2 & 0 & 1 & 2 \\
\hline & & & $\begin{array}{l}\text { 24. Joins ongoing activity of group without being sold } \\
\text { to do so. }\end{array}$ & 0 & 1 & 2 & 0 & 1 & 2 \\
\hline & & & $\begin{array}{l}\text { 25. Fesponds appropriately when pushed or hit by } \\
\text { other chldren. }\end{array}$ & 0 & 1 & 2 & 0 & 1 & 2 \\
\hline & & & 26. Ignores peer distractions when doing class work. & 0 & 1 & 2 & 0 & 1 & 2 \\
\hline & & & 27. Keeps desk clean and neat without being reminded. & D & 1 & 2 & 0 & 1 & 2 \\
\hline & & & 28. Attends to your instnuctiona. & 0 & 1 & 2 & 0 & 1 & 2 \\
\hline & & & $\begin{array}{l}\text { 29. Easily makes transition from one classroom activity } \\
\text { to andher. }\end{array}$ & 0 & 1 & 2 & 0 & 1 & 2 \\
\hline & & & 30. Gets along with pecple who are diflerent. & 0 & 1 & 2 & 0 & 1 & 2 \\
\hline c & A & 5 & Wes or HOW ORTEN COUNAS & & & & & & \\
\hline
\end{tabular}

\begin{tabular}{|c|c|c|c|c|c|c|}
\hline \multicolumn{3}{|c|}{ 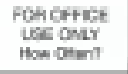 } & \multirow[t]{2}{*}{ Problem Behaviors } & \multirow{2}{*}{\multicolumn{2}{|c|}{$\begin{array}{c}\text { How } \\
\text { Othern? } \\
\text { Sartinat }\end{array}$}} & \multirow[b]{2}{*}{$\begin{array}{l}\text { Very } \\
\text { onter }\end{array}$} \\
\hline E & 1 & $\mathrm{H}$ & & & & \\
\hline & & & 31. Fights with cthers. & 0 & 1 & 2 \\
\hline & & & 32. Has bw self-esteem. & 0 & 1 & 2 \\
\hline & & & 33. Threatnns or bulles otherg. & 0 & 1 & 2 \\
\hline & & & 34. Appoars lanely. & 0 & 1 & 2 \\
\hline & & & 35. Is easily distracted. & 0 & 1 & 2 \\
\hline & & & 36. Internupts cormersations of others. & 0 & 1 & 2 \\
\hline & & & 37. Dishurbs ongoing activities. & 0 & 1 & 2 \\
\hline & & & 38. Shows anxiety about being with a group of children. & 0 & 1 & 2 \\
\hline & & & 39. Is easily embarrassed. & 0 & 1 & 2 \\
\hline & & & 40. Donsnt liston to what others say. & 0 & 1 & 2 \\
\hline & & & 41. Argues with others. & $\mathbf{0}$ & 1 & 2 \\
\hline & & & 42. Talks back to aduhs when corrected. & 0 & 1 & 2 \\
\hline & & & 43. Gets angry easily. & 0 & 1 & 2 \\
\hline & & & 44. Has bemper tantrums. & 0 & 1 & 2 \\
\hline & & & 45. Lilkes to be alane. & 0 & 1 & 2 \\
\hline & & & 46. Acts sad or dopressed. & 0 & 1 & 2 \\
\hline & & & 47. Acts impulshely. & 0 & 1 & 2 \\
\hline & & & 48. Fidgets or moves encessivelf. & 0 & 1 & 2 \\
\hline$\varepsilon$ & 1 & $\mathrm{H}$ & BUS OF HOE OPTEN COUNAS & & & \\
\hline
\end{tabular}

\section{Do not make} imporance ratroge for iens $31=48$

Go on to

Page 4. 


\section{Academic Competence}

The naxt nine inems require your judgments of this student's acadomic or learning behaviors as observed in your classroom. Compare the student with other children whe are in the same dassroort.

Rate all hems using a scale of 1 to 5 . Cincle the number that best represents your judgment. The number 1 indicates the lowest or least favorable pertormance, placing the student in the lowest $10 \%$ of the class. Number $\mathbf{5}$ indicates the highest or most favorable pertomance, placing the studont in the highast $10 \%$ compared with other students in the classroom.

\begin{tabular}{|c|c|c|c|c|c|c|c|}
\hline \multirow[t]{2}{*}{$\begin{array}{c}\text { mon } \\
\text { ontoe } \\
\text { Ued } \\
\text { cher }\end{array}$} & & & Lowen: & $\begin{array}{l}\text { Noot Lovest } \\
20 \%\end{array}$ & Mode & $\begin{array}{l}\text { Wax Hones: } \\
\text { 20\% }\end{array}$ & Host \\
\hline & & $\begin{array}{l}\text { Compared with other children in my classioom, the } \\
\text { overall academic performance of this child is: }\end{array}$ & 1 & 2 & 3 & 4 & 5 \\
\hline 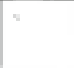 & 50. & $\begin{array}{l}\text { In reading, how does this child compare with } \\
\text { ather students? }\end{array}$ & 1 & 2 & 3 & 4 & 5 \\
\hline & & $\begin{array}{l}\text { In mathematics, how does this child compere } \\
\text { with other students? }\end{array}$ & 1 & 2 & 3 & 4 & 5 \\
\hline & 52. & $\begin{array}{l}\text { In terms of grade-level expectations, this child's } \\
\text { skils in reading are: }\end{array}$ & 1 & 2 & 3 & 4 & 5 \\
\hline & 53. & $\begin{array}{l}\text { In terms of grade-level expectatians, this child's } \\
\text { skills in mathematics are: }\end{array}$ & 1 & 2 & 3 & 4 & 5 \\
\hline & & $\begin{array}{l}\text { This chlid's overall motivation to succeed } \\
\text { academically is: }\end{array}$ & 1 & 2 & 3 & 4 & 5 \\
\hline & & $\begin{array}{l}\text { This child's parental encouragement to succeed } \\
\text { acaderrically is: }\end{array}$ & 1 & 2 & 3 & 4 & 5 \\
\hline & & $\begin{array}{l}\text { Compared with other children in my classroom } \\
\text { this childs intellectual functioning is: }\end{array}$ & 1 & 2 & 3 & 4 & 5 \\
\hline & & $\begin{array}{l}\text { Comparbd with other children in my classroom } \\
\text { this childs overall classroom behavior is: }\end{array}$ & 1 & 2 & 3 & 4 & 5 \\
\hline
\end{tabular}

a sus co counas

Stop. Please check to be gure all litems have been marked.

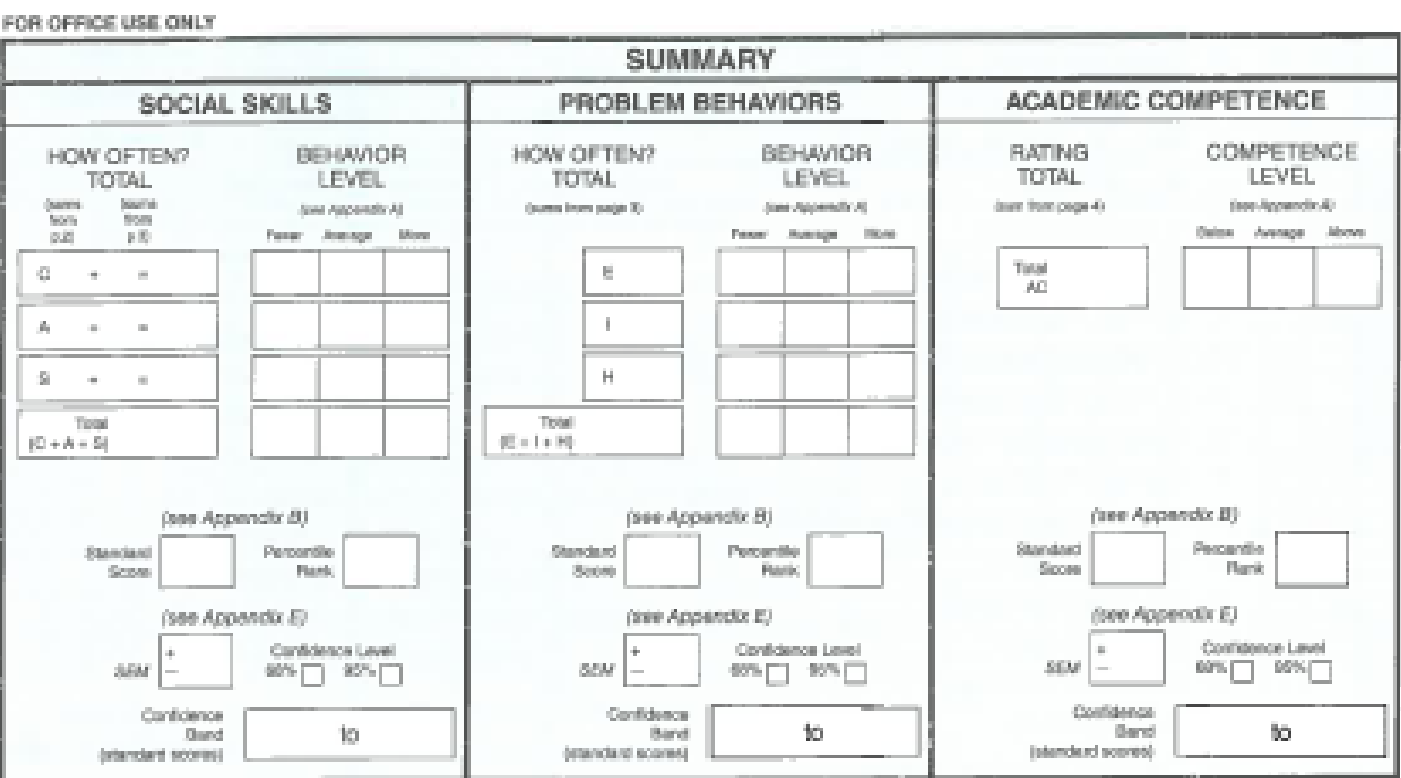

Noms ued Mandeapsed Noetaribicippod

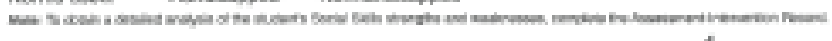




\section{WRAT4}

\section{BLUE RESPONSE FORM}

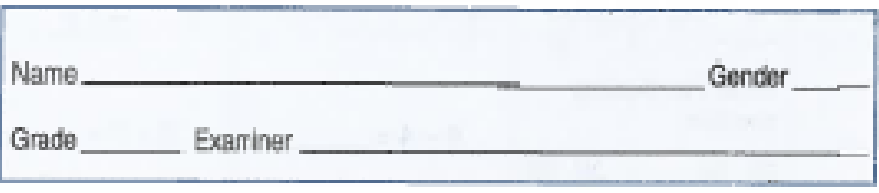

\begin{tabular}{|c|c|c|c|}
\hline & Yoar & Month & Day \\
\hline \multicolumn{4}{|l|}{ Dwe of Test } \\
\hline \multicolumn{4}{|l|}{ Deste *f Brth } \\
\hline Age & & & \\
\hline
\end{tabular}

\section{SPELLING SUBTEST}

Pert 1: Letter Writing

Write Name

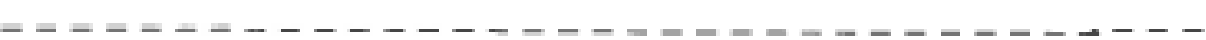

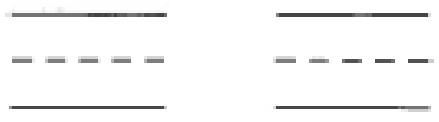

(3)

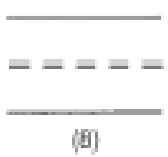

(8)

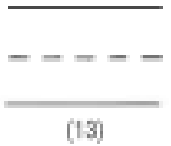

(13)
(4)

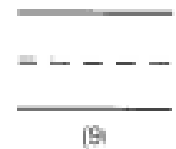

(9)

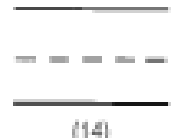

$(14)$

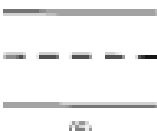

(5)

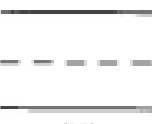

$(10)$

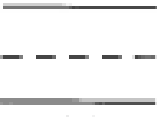

(15)

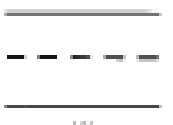

$(6$.

$(7)$

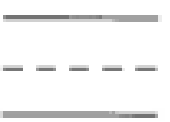

(11)
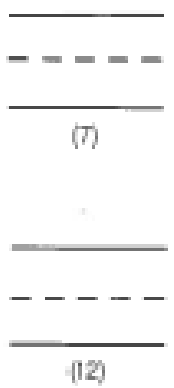

(12)

Latter Writing Raw Score

[continue to Part $z$ : Speling on page 4)
QR WIDE RANGE

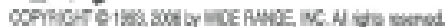

987454
Retulad by

PAR $16204 \mathrm{~N}$. Florida twe, * Lutz, FL 33549 nawar. 1.800.331.8378 - wWw. parinc,com 


\section{MATH COMPUTATION SUBTEST}

Part 2: Math Computation

Wite all answers in simplest form

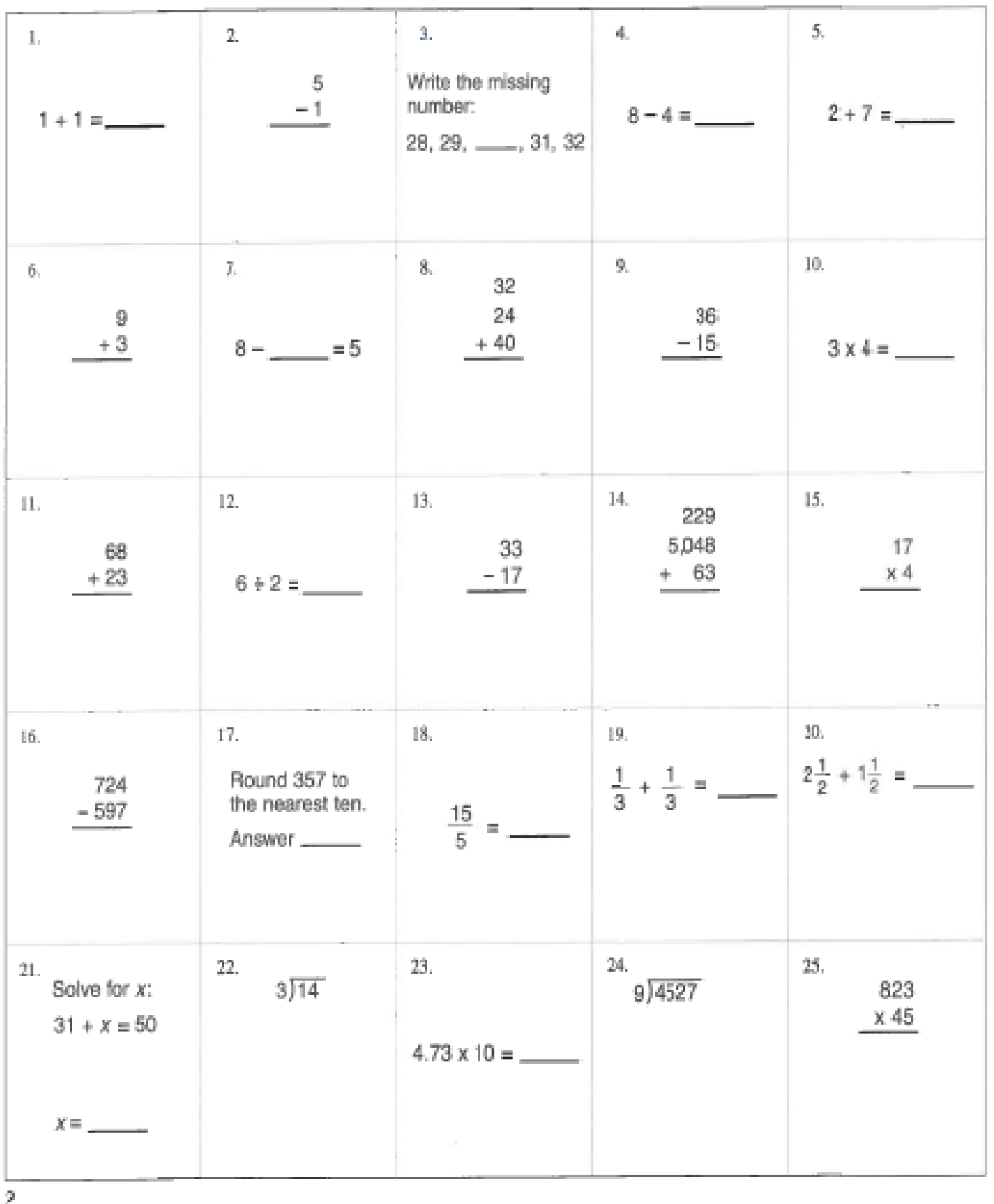


Part 2: Math Computation

Write all answers in simplest form
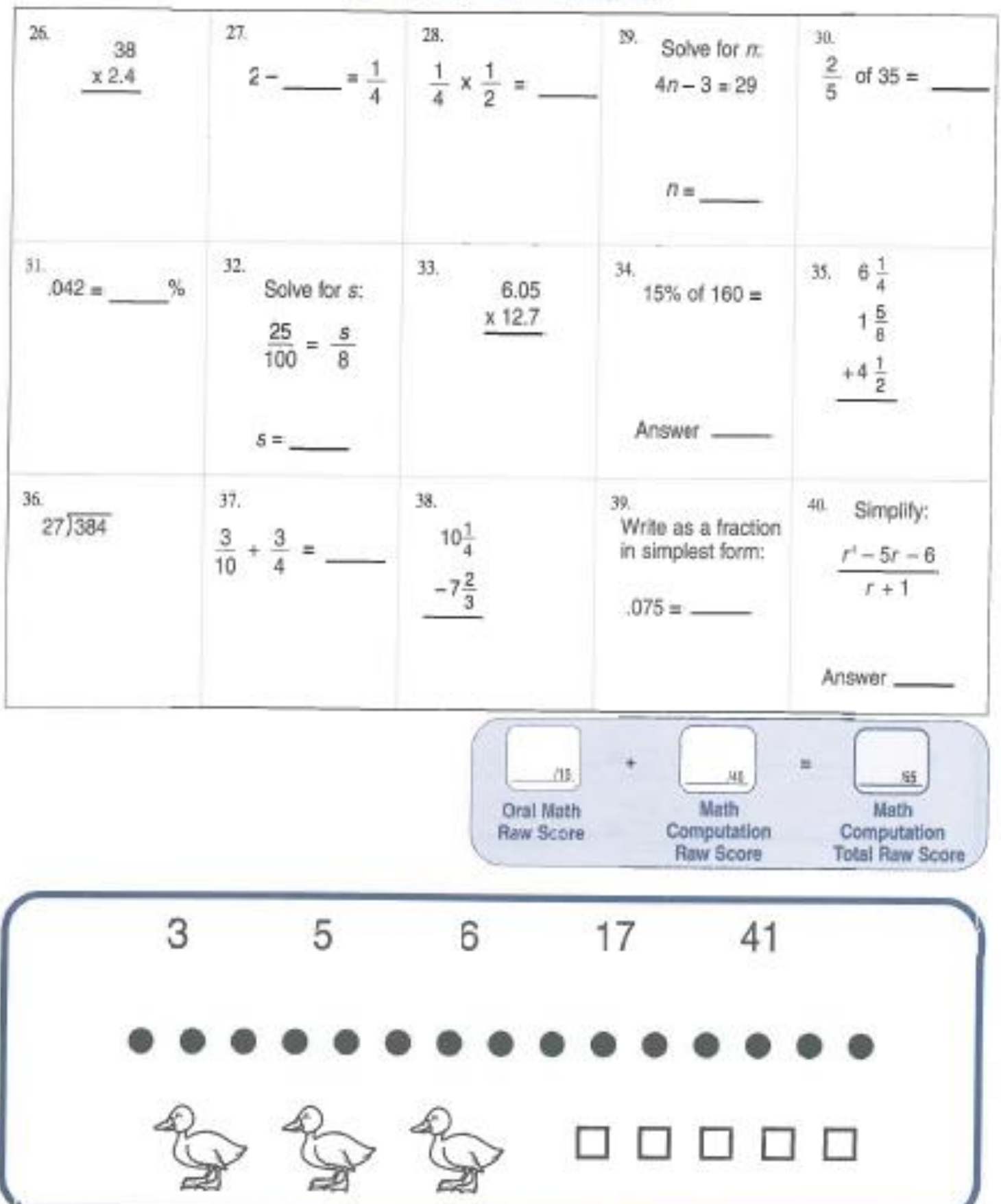


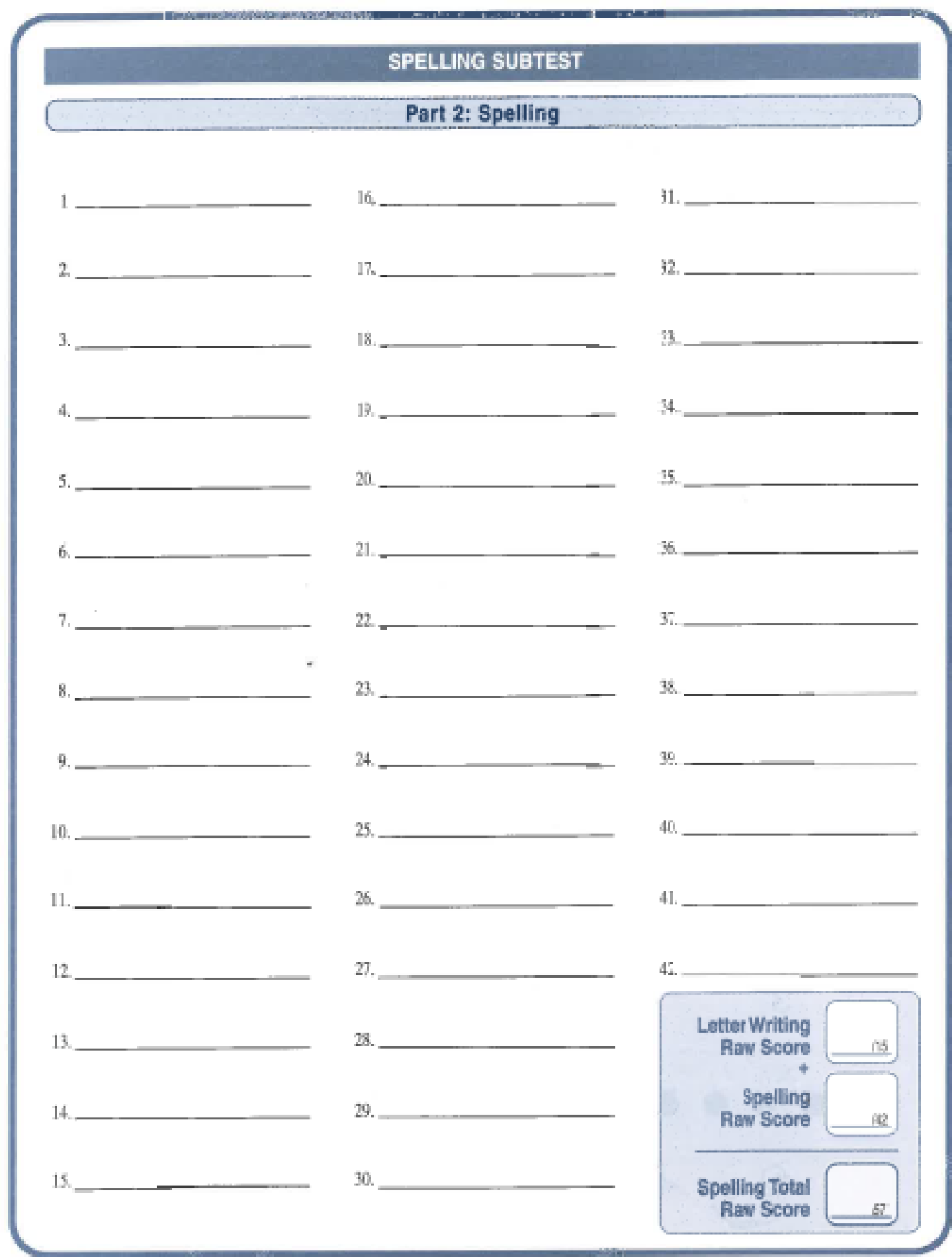

